\title{
Mod- Convergence, II: Estimates on the Speed of Convergence
}

\author{
Féray, Valentin ; Méliot, Pierre-Loïc ; Nikeghbali, Ashkan
}

\begin{abstract}
In this paper, we give estimates for the speed of convergence towards a limiting stable law in the recently introduced setting of mod- convergence. Namely, we define a notion of zone of control, closely related to mod- convergence, and we prove estimates of Berry-Esseen type under this hypothesis. Applications include: - the winding number of a planar Brownian motion; - classical approximations of stable laws by compound Poisson laws; - examples stemming from determinantal point processes (characteristic polynomials of random matrices and zeroes of random analytic functions); - sums of variables with an underlying dependency graph (for which we recover a result of Rinott, obtained by Stein's method); - the magnetization in the d-dimensional Ising model; - and functionals of Markov chains.
\end{abstract}

DOI: https://doi.org/10.1007/978-3-030-28535-7_15

Posted at the Zurich Open Repository and Archive, University of Zurich

ZORA URL: https://doi.org/10.5167/uzh-180249

Book Section

Submitted Version

Originally published at:

Féray, Valentin; Méliot, Pierre-Loïc; Nikeghbali, Ashkan (2019). Mod- Convergence, II: Estimates on the Speed of Convergence. In: Donati-Martin, Catherine; Lejay, Antoine; Rouault, Alain. Séminaire de Probabilités, L. Cham, Switzreland: Springer, 405-477.

DOI: https://doi.org/10.1007/978-3-030-28535-7_15 


\title{
Mod- $\phi$ convergence, II: Estimates on the speed of convergence
}

\author{
Valentin Féray, Pierre-Loïc Méliot, and Ashkan Nikeghbali
}

\begin{abstract}
In this paper, we give estimates for the speed of convergence towards a limiting stable law in the recently introduced setting of mod- $\phi$ convergence. Namely, we define a notion of zone of control, closely related to mod- $\phi$ convergence, and we prove estimates of Berry-Esseen type under this hypothesis. Applications include:

- the winding number of a planar Brownian motion;

- classical approximations of stable laws by compound Poisson laws;

- examples stemming from determinantal point processes (characteristic polynomials of random matrices and zeroes of random analytic functions);

- sums of variables with an underlying dependency graph (for which we recover a result of Rinott, obtained by Stein's method);

- the magnetization in the $d$-dimensional Ising model;

- and functionals of Markov chains.
\end{abstract}

\section{Introduction}

\subsection{Mod- $\phi$ convergence}

Let $\left(X_{n}\right)_{n \in \mathbb{N}}$ be a sequence of real-valued random variables. In many situations, there exists a scale $s_{n}$ and a limiting law $\phi$ which is infinitely divisible, such that $\left(X_{n} / s_{n}\right)_{n \in \mathbb{N}}$ converges in law towards $\phi$. For instance, in the classical central limit theorem, if $X_{n}=\sum_{i=1}^{n} A_{i}$ is a sum of centered i.i.d. random variables with $\mathbb{E}\left[\left(A_{1}\right)^{2}\right]<\infty$, then

$$
s_{n}=\sqrt{n \mathbb{E}\left[\left(A_{1}\right)^{2}\right]}
$$

and the limit is the standard Gaussian distribution $\mathcal{N}_{\mathbb{R}}(0,1)$. In [JKN11] and the subsequent papers [DKN15, FMN16], the notion of mod- $\phi$ convergence was developed in order to get quantitative estimates on the convergence $\frac{X_{n}}{s_{n}} \rightarrow \phi$ (throughout the paper, $\rightarrow$ denotes convergence in distribution).

Definition 1. Let $\phi$ be an infinitely divisible probability measure, and $D \subset \mathbb{C}$ be a subset of the complex plane, which we assume to contain 0. We assume that the Laplace transform of $\phi$ is well defined over $D$, with Lévy exponent $\eta$ :

$$
\forall z \in D, \quad \int_{\mathbb{R}} \mathrm{e}^{z x} \phi(d x)=\mathrm{e}^{\eta(z)} .
$$

We then say that $\left(X_{n}\right)_{n \in \mathbb{N}}$ converges mod- $\phi$ over $D$, with parameters $\left(t_{n}\right)_{n \in \mathbb{N}}$ and limiting function $\psi: D \rightarrow \mathbb{C}$, if $t_{n} \rightarrow+\infty$ and if, locally uniformly on $D$, 


$$
\lim _{n \rightarrow \infty} \mathbb{E}\left[\mathrm{e}^{z X_{n}}\right] \mathrm{e}^{-t_{n} \eta(z)}=\psi(z) .
$$

If $D=i \mathbb{R}$, we shall just speak of mod- $\phi$ convergence; it is then convenient to use the notation

$$
\begin{aligned}
\theta_{n}(\xi) & =\mathbb{E}\left[\mathrm{e}^{\mathrm{i} \xi X_{n}}\right] \mathrm{e}^{-t_{n} \eta(\mathrm{i} \xi)} ; \\
\theta(\xi) & =\psi(\mathrm{i} \xi),
\end{aligned}
$$

so that mod- $\phi$ convergence corresponds to $\lim _{n \rightarrow \infty} \theta_{n}(\xi)=\theta(\xi)$ (uniformly for $\xi$ in compact subsets of $\mathbb{R}$ ). When nothing is specified, in this paper, we implicitly consider that $D=\mathbb{i} \mathbb{R}$. When $D=\mathbb{C}$ we shall speak of complex mod- $\phi$ convergence. In some situations, it is also appropriate to study mod- $\phi$ convergence on a band $\mathbb{R} \times \mathrm{i}[-b, b]$, or $[-c, c] \times \mathrm{i} \mathbb{R}($ see $[\mathrm{FMN16}, \mathrm{MN} 15])$.

Intuitively, a sequence of random variables $\left(X_{n}\right)_{n \in \mathbb{N}}$ converges mod- $\phi$ if it can be seen as a large renormalization of the infinitely divisible law $\phi$, plus some residue which is asymptotically encoded in the Fourier or Laplace sense by the limiting function $\psi$. Then, $\phi$ will typically be:

1. in the case of lattice-valued distributions, a Poisson law or a compound Poisson law ( $c f$. [BKN09, KN10, FMN16, CDMN15]);

2. or, a stable distribution, for instance a Gaussian law.

In this paper, we shall only be interested in the second case. Background on stable distributions is given at the end of this introduction (Section 1.3). In particular we will see that, if $\phi$ is a stable distribution, then the mod- $\phi$ convergence of $X_{n}$ implies the convergence in distribution of a renormalized version $Y_{n}$ of $X_{n}$ to $\phi$ (Proposition 3).

We believe that mod- $\phi$ is a kind of universality class behind the central limit theorem (or its stable version) in the following sense. For many sequences $\left(X_{n}\right)_{n \in \mathbb{N}}$ of random variables that are proven to be asymptotically normal (or converging to a stable distribution), it is possible to prove mod- $\phi$ convergence; we refer to our monograph [FMN16] or Sections 3-5 below for such examples. These estimates on the Laplace transform/characteristic function can then be used to derive in an automatic way some companion theorems, refining the central limit theorem. In [FMN16], we discuss in details the question of moderate/large deviation estimates and of finding the normality zone.

In the present paper, we shall be interested in the speed of convergence towards the Gaussian (or more generally the stable) distribution of the appropriate renormalization $Y_{n}$ of $X_{n}$. To obtain sharp bounds on this speed of convergence, we do not work with mod- $\phi$ convergence, but we introduce the notion of $z$ one of control for the renormalized characteristic function $\theta_{n}(\xi)$. In many examples, such a zone of control can be obtained by essentially the same arguments used to prove mod- $\phi$ convergence, and in most examples, mod- $\phi$ convergence actually holds.

\subsection{Results and outline of the paper}

We take as reference law a stable distribution $\phi$ of index $\alpha \in(0,2]$. Let $\left(X_{n}\right)_{n \in \mathbb{N}}$ be a sequence of variables that admits a zone of control (this notion will be defined in Definition 5 ; this is closely related to the mod- $\phi$ convergence of $\left.\left(X_{n}\right)_{n \in \mathbb{N}}\right)$. As we will see in Proposition 6 , this implies that some renormalization $Y_{n}$ of $X_{n}$ converges in distribution towards $\phi$ and we are interested in the speed of convergence for this convergence. More precisely, we are interested in upper bounds for the Kolmogorov distance

$$
d_{\mathrm{Kol}}\left(Y_{n}, \phi\right)=\sup _{a \in \mathbb{R}}\left|\mathbb{P}\left[Y_{n} \leq a\right]-\int_{-\infty}^{a} \phi(d x)\right| .
$$

The main theorem of Section 2 (Theorem 20) shows that this distance is $\mathcal{O}\left(t_{n}^{-\gamma-1 / \alpha}\right)$, where $\gamma$ is a parameter describing how large our zone of control is. We also obtain as intermediate result estimates for 


$$
\left|\mathbb{E}\left[f\left(Y_{n}\right)\right]-\int_{\mathbb{R}} f(x) \phi(d x)\right|,
$$

where $f$ lies in some specific set of tests functions (Proposition 16). A detailed discussion on the method of proof of these bounds can be found at the beginning of Section 2 .

Section 3 gives some examples of application of the theoretical results of Section 2. The first one is a toy example, while the other ones are new to the best of our knowledge.

- We first consider sums of i.i.d. random variables with finite third moment. In this case, the classical Berry-Esseen estimate ensures that

$$
d_{\mathrm{Kol}}\left(Y_{n}, \mathcal{N}_{\mathbb{R}}(0,1)\right) \leq \frac{3 \mathbb{E}\left[\left|A_{1}\right|^{3}\right]}{\sigma^{3} \sqrt{n}},
$$

see [Ber41] or [Fel71, §XVI.5, Theorem 1]. Our general statement for variables with a zone of convergence gives essentially the same result, only the constant factor is not as good.

- We can extend the Berry-Esseen estimates to the case of independent but non identically distributed random variables. As an example, we look at the number of zeroes $Z_{r}$ of a random analytic series that fall in a disc of radius $r$; it has the same law as a series of independent Bernoulli variables of parameters $r^{2 k}, k \geq 1$. When the radius $r$ of the disc goes to 1 , one has a central limit theorem for $Z_{r}$, and the theory of zones of control yields an estimate $O\left((1-r)^{-1 / 2}\right)$ on the Kolmogorov distance.

- We then look at the winding number $\varphi_{t}$ of a planar Brownian motion starting at 1 (see Section 3.2 for a precise definition). This quantity has been proven to converge in the mod-Cauchy sense in [DKN15], based on the computation of the characteristic function done by Spitzer [Spi58]. The same kind of argument easily yields the existence of a zone of control and our general result applies: when $t$ goes to infinity, after renormalization, $\varphi_{t}$ converges in distribution towards a Cauchy law and the Kolmogorov distance in this convergence is $\mathcal{O}\left((\log t)^{-1}\right)$.

- In the third example, we consider compound Poisson laws (see [Sat99, Chapter 1, §4]). These laws appear in the proof of the Lévy-Khintchine formula for infinitely divisible laws (loc. cit., Chapter 2, $\S 8$, p. 44-45), and we shall be interested in those that approximate the stable distributions $\phi_{c, \alpha, \beta}$. Again, establishing the existence of a zone of control is straight-forward and our general result shows that the speed of convergence is $\mathcal{O}\left(n^{-1 / \min (\alpha, 1)}\right)$ (Proposition 23), with an additional log factor if $\alpha=1$ and $\beta \neq 0$ (thus exhibiting an interesting phase transition phenomenon).

- Ratios of Fourier transforms of probability measures appear naturally in the theory of self-decomposable laws and of the corresponding Ornstein-Uhlenbeck processes. Thus, any self-decomposable law $\phi$ is the limiting distribution of a Markov process $\left(U_{t}\right)_{t \geq 0}$, and when $\phi$ is a stable law, one has mod- $\phi$ convergence of an adequate renormalisation of $U_{t}$, with a constant residue. This leads to an estimate of the speed of convergence which depends on $\alpha$, on the speed of $\left(U_{t}\right)_{t \geq 0}$ and on its starting point (Proposition 25).

- Finally, logarithms of characteristic polynomials of random matrices in a classical compact Lie group are mod-Gaussian convergent (see for instance [FMN16, Section 7.5]), and one can compute a zone of control for this convergence, which yields an estimate of the speed of convergence $O\left((\log n)^{-3 / 2}\right)$. For unitary groups, one recovers [BHNY08, Proposition 5.2]. This example shows how one can force the index $v$ of a zone of control of mod-Gaussian convergence to be equal to 3, see Remark 26 .

The last two sections concentrate on the case where the reference law is Gaussian $(\alpha=2)$. In this case, we show that a sufficient condition for having a zone of control is to have uniform bounds on cumulants (see Definition 28 and Lemma 29). This is not surprising since such bounds are known to imply (with small additional hypotheses) mod-Gaussian convergence [FMN16, Section 5.1]. Combined with our main result, this gives bounds for the Kolmogorov distance for variables having uniform bounds on cumulants - see Corollary 30. Note nevertheless that similar results have been given previously by Statulevičius [Sta66] (see also Saulis and Statulevičius [SS91]). Our Corollary 30 coincides up to a constant factor 
to one of their result. Our contribution here therefore consists in giving a large variety of non-trivial examples where such bounds on cumulants hold:

- The first family of examples relies on a previous result by the authors [FMN16, Chapter 9] (see Theorem 34 here), where bounds on cumulants for sums of variables with an underlying dependency graph are given. Let us comment a bit. Though introduced originally in the framework of the probabilistic method [AS08, Chapter 5], dependency graphs have been used to prove central limit theorems on various objects: random graphs [Jan88], random permutations [Bón10], probabilistic geometry [PY05], random character values of the symmetric group [FMN16, Chapter 11]. In the context of Stein's method, we can also obtain bounds for the Kolmogorov distance in these central limit theorems [BR89, Rin94].

The results of this paper give another approach to obtain bounds for this Kolmogorov distance for sums of bounded variables (see Section 4.2). The bounds obtained are, up to a constant, the same as in [Rin94, Theorem 2.2]. Note that our approach is fundamentally different, since it relies on classical Fourier analysis, while Stein's method is based on a functional equation for the Gaussian distribution. We make these bounds explicit in the case of subgraph counts in Erdös-Rényi random graphs and discuss an extension to sum of unbounded variables.

- The next example is the finite volume magnetization in the Ising model on $\mathbb{Z}^{d}$. The Ising model is one of the most classical models of statistical mechanics, we refer to [FV16] and references therein for an introduction to this vast topic. The magnetization $M_{\Delta}$ (that is the sum of the spins in $\Delta$ ) is known to have asymptotically normal fluctuations [New80]. Based on a result of Duneau, Iagolnitzer and Souillard [DIS74], we prove that, if the magnetic field is non-zero or if the temperature is sufficiently large, $M_{\Delta}$ has uniform bounds on cumulants. This implies a bound on the Kolmogorov distance (Proposition 49):

$$
d_{\text {Kol }}\left(\frac{M_{\Delta}-\mathbb{E}\left[M_{\Delta}\right]}{\sqrt{\operatorname{Var}\left(M_{\Delta}\right)}}, \mathcal{N}_{\mathbb{R}}(0,1)\right) \leq \frac{K}{\sqrt{|\Delta|}} .
$$

It seems that this result improves on what is known so far. In [Bul96], Bulinskii gave a general bound on the Kolmogorov distance for sums of associated random variables, which applied to $M_{\Delta}$, yields a bound with an additional $(\log |\Delta|)^{d}$ factor comparing to ours. In a slightly different direction, Goldstein and Wiroonsri [GW16] have recently given a bound of order $\mathcal{O}\left(|\Delta|^{1 /(2 d+2)}\right)$ for the $\mathrm{L}^{1}$ distance (the $\mathrm{L}^{1}$-distance is another distance on distribution functions, which is a priori incomparable with the Kolmogorov distance; note also that their bound is only proved in the special case where $\left.\Delta=\{-m,-m+1, \ldots, m\}^{d}\right)$.

- The last example considers statistics of the form $S_{n}=\sum_{t=0}^{n} f_{t}\left(X_{t}\right)$, where $\left(X_{t}\right)_{t \geq 0}$ is an ergodic discrete time Markov chain on a finite space state. Again we can prove uniform bounds on cumulants and deduce from it bounds for the Kolmogorov distance (Theorem 55). The speed of convergence in the central limit theorem for Markov chains has already been studied by Bolthausen [Bol80] (see also later contributions of Lezaud [Lez96] and Mann [Man96]). These authors study more generally Markov chains on infinite space state, but focus on the case of a statistics $f_{t}$ independent of the time. Except for these differences, the bounds obtained are of the same order; however our approach and proofs are again quite different.

It is interesting to note that the proofs of the bounds on cumulants in the last two examples are highly non trivial and share some common structure. Each of these statistics decomposes naturally as a sum. In each case, we give an upper bound for joint cumulants of the summands, which writes as a weighted enumeration of spanning trees. Summing terms to get a bound on the cumulant of the sum is then easy.

To formalize this idea, we introduce in Section 5 the notion of uniform weighted dependency graphs. Both proofs for the bounds on cumulants (for magnetization of the Ising model and functional of Markov chains) are presented in this framework. We hope that this will find further applications in the future. 


\subsection{Stable distributions and mod-stable convergence}

Let us recall briefly the classification of stable distributions (see [Sat99, Chapter 3]). Fix $c>0$ (the scale parameter), $\alpha \in(0,2]$ (the stability parameter), and $\beta \in[-1,1]$ (the skewness parameter).

Definition 2. The stable distribution of parameters $(c, \alpha, \beta)$ is the infinitely divisible law $\phi=\phi_{c, \alpha, \beta}$ whose Fourier transform

$$
\widehat{\phi}(\xi)=\int_{\mathbb{R}} \mathrm{e}^{\mathrm{i} x \xi} \phi(d x)=\mathrm{e}^{\eta(\mathrm{i} \xi)}
$$

has for Lévy exponent $\eta(\mathrm{i} \xi)=\eta_{c, \alpha, \beta}(\mathrm{i} \xi)=-|c \xi|^{\alpha}(1-\mathrm{i} \beta h(\alpha, \xi) \operatorname{sgn}(\xi))$, where

$$
h(\alpha, \xi)= \begin{cases}\tan \left(\frac{\pi \alpha}{2}\right) & \text { if } \alpha \neq 1, \\ -\frac{2}{\pi} \log |\xi| & \text { if } \alpha=1\end{cases}
$$

and $\operatorname{sgn}(\xi)= \pm 1$ is the sign of $\xi$.

The most usual stable distributions are:

- the standard Gaussian distribution $\frac{1}{\sqrt{2 \pi}} \mathrm{e}^{-x^{2} / 2} d x$ for $c=\frac{1}{\sqrt{2}}, \alpha=2$ and $\beta=0$;

- the standard Cauchy distribution $\frac{1}{\pi\left(1+x^{2}\right)} d x$ for $c=1, \alpha=1$ and $\beta=0$;

- the standard Lévy distribution $\frac{1}{\sqrt{2 \pi}} \frac{\mathrm{e}^{-1 / 2 x}}{x^{3 / 2}} \mathbb{1}_{x \geq 0} d x$ for $c=1, \alpha=\frac{1}{2}$ and $\beta=1$.
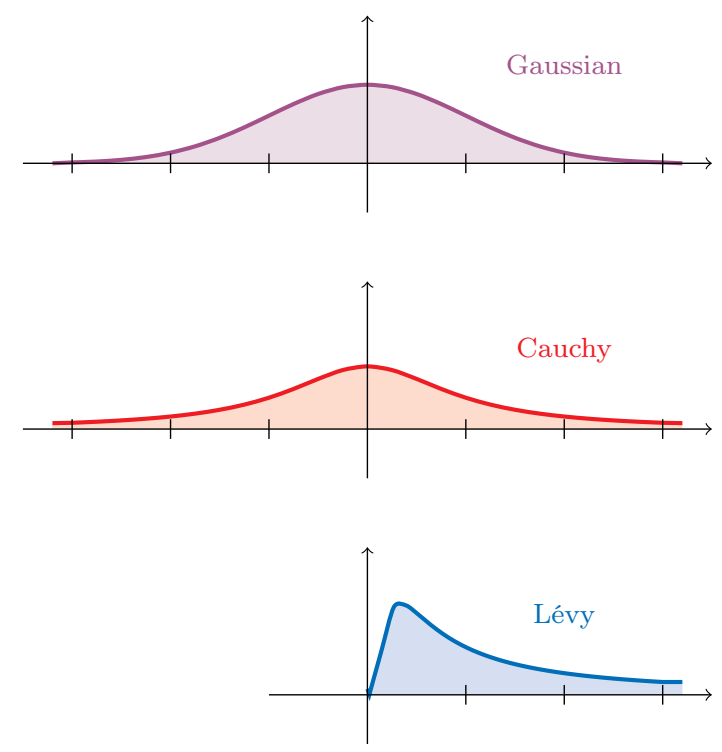

Fig. 1 Densities of the standard Gaussian, Cauchy and Lévy distribution.

We recall that mod- $\phi$ convergence on an open subset $D$ of $\mathbb{C}$ containing 0 can only occur when the characteristic function of $\phi$ is analytic around 0. Among stable distributions, only Gaussian laws (which correspond to $\alpha=2$ ) satisfy this property. Mod- $\phi$ convergence on $D=i \mathbb{R}$ can however be considered for any stable distribution $\phi$.

Since $\left|\mathrm{e}^{\eta(\mathrm{i} \xi)}\right|=\mathrm{e}^{-|c \xi|^{\alpha}}$ is integrable, any stable law $\phi_{c, \alpha, \beta}$ has a density $m_{c, \alpha, \beta}(x) d x$ with respect to the Lebesgue measure. Moreover, the corresponding Lévy exponents have the following scaling property: for any $t>0$, 


$$
t \eta_{c, \alpha, \beta}\left(\frac{\mathrm{i} \xi}{t^{1 / \alpha}}\right)= \begin{cases}\eta_{c, \alpha, \beta}(\mathrm{i} \xi) & \text { if } \alpha \neq 1, \\ \eta_{c, \alpha, \beta}(\mathrm{i} \xi)-\left(\frac{2 c \beta}{\pi} \log t\right) \mathrm{i} \xi & \text { if } \alpha=1 .\end{cases}
$$

This will be used in the following proposition:

Proposition 3. If $\left(X_{n}\right)_{n \in \mathbb{N}}$ converges in the $\bmod -\phi_{c, \alpha, \beta}$ sense, then

$$
Y_{n}= \begin{cases}\frac{X_{n}}{\left(t_{n}\right)^{1 / \alpha}} & \text { if } \alpha \neq 1, \\ \frac{X_{n}}{t_{n}}-\frac{2 c \beta}{\pi} \log t_{n} & \text { if } \alpha=1\end{cases}
$$

converges in law towards $\phi_{c, \alpha, \beta}$.

Proof. In both situations,

$$
\mathbb{E}\left[\mathrm{e}^{\mathrm{i} \xi Y_{n}}\right]=\mathrm{e}^{\eta(\mathrm{i} \xi)} \theta_{n}\left(\frac{\xi}{\left(t_{n}\right)^{1 / \alpha}}\right)=\mathrm{e}^{\eta(\mathrm{i} \xi)} \theta(0)(1+o(1))=\mathrm{e}^{\eta(\mathrm{i} \xi)}(1+o(1))
$$

thanks to the uniform convergence of $\theta_{n}$ towards $\theta$, and to the scaling property of the Lévy exponent $\eta$.

\section{Speed of convergence estimates}

The goal of this section is to introduce the notion of zone of control (Section 2.1) and to estimate the speed of convergence in the resulting central limit theorem. More precisely, we take as reference law a stable distribution $\phi_{c, \alpha, \beta}$ and a sequence $\left(X_{n}\right)_{n \in \mathbb{N}}$ that admits a zone of control (with respect to $\left.\phi_{c, \alpha, \beta}\right)$. As for mod- $\phi_{c, \alpha, \beta}$ convergent sequences, it is easy to prove that in this framework, an appropriate renormalization $Y_{n}$ of $X_{n}$ converges in distribution towards $\phi_{c, \alpha, \beta}$ (see Proposition 6 below).

If $Y$ has distribution $\phi_{c, \alpha, \beta}$, we then want to estimate

$$
d_{\mathrm{Kol}}\left(Y_{n}, Y\right)=\sup _{s \in \mathbb{R}}\left|\mathbb{P}\left[Y_{n} \leq s\right]-\mathbb{P}[Y \leq s]\right| .
$$

To do this, we follow a strategy proposed by Tao (see [Tao12, Section 2.2]) in the case of sums of i.i.d. random variables with finite third moment. The right-hand side of (1) can be rewritten as

$$
\sup _{f \in \mathcal{F}}\left|\mathbb{E}\left[f\left(Y_{n}\right)\right]-\mathbb{E}[f(Y)]\right|,
$$

where $\mathcal{F}$ is the class of measurable functions $y \mapsto 1_{y \leq s}$. Therefore, it is natural to approach the problem of speed of convergence by looking at general estimates on test functions. The basic idea is then to use the Parseval formula to compute the difference $\mathbb{E}\left[f\left(Y_{n}\right)\right]-\mathbb{E}[f(Y)]$, since we have estimates on the Fourier transforms of $Y_{n}$ and $Y$. A difficulty comes from the fact that the functions $y \mapsto 1_{y \leq s}$ are not smooth, and in particular, their Fourier transforms are only defined in the sense of distributions. This caveat is dealt with by standard techniques of harmonic analysis (Sections 2.2 to 2.4): namely, we shall work in a space of distributions instead of functions, and use an adequate smoothing kernel in order to be able to work with compactly supported Fourier transforms. Section 2.5 gathers all these tools to give an upper bound for (1). This is the main result of this section and can be found in Theorem 20 .

Remark 4. An alternative way to get an upper bound for (1) from estimates on characteristic functions is to use the following inequality due to Berry (see [Ber41] or [Fel71, Lemma XVI.3.2]). Let $X$ and $Y$ be random variables with characteristic functions $f^{*}(\zeta)$ and $g^{*}(\zeta)$. Then, provided that $Y$ has a density bounded by $m$, we have, for any $s \in \mathbb{R}$,

$$
|\mathbb{P}[X \leq s]-\mathbb{P}[Y \leq s]| \leq \frac{1}{\pi} \int_{-T}^{T}\left|\frac{f^{*}(\zeta)-g^{*}(\zeta)}{\zeta}\right| d \zeta+\frac{24 m}{\pi T} .
$$


Using this inequality in our context should lead to similar estimates as the ones we obtain, possibly with different constants. The proof we use here however has the advantage of being more self-contained, and to provide estimates for test functions as intermediate results.

\subsection{The notion of zone of control}

Definition 5. Let $\left(X_{n}\right)_{n \in \mathbb{N}}$ be a sequence of real random variables, $\phi_{c, \alpha, \beta}$ a reference stable law, and $\left(t_{n}\right)_{n \in \mathbb{N}}$ a sequence growing to infinity. Consider the following assertions:

(Z1) Fix $v>0, w>0$ and $\gamma \in \mathbb{R}$. There exists a zone $\left[-K\left(t_{n}\right)^{\gamma}, K\left(t_{n}\right)^{\gamma}\right]$ such that, for all $\xi$ in this zone, if $\theta_{n}(\xi)=\mathbb{E}\left[\mathrm{e}^{\mathrm{i} \xi X_{n}}\right] \mathrm{e}^{-t_{n} \eta_{c, \alpha, \beta}(\mathrm{i} \xi)}$, then

$$
\left|\theta_{n}(\xi)-1\right| \leq K_{1}|\xi|^{v} \exp \left(K_{2}|\xi|^{w}\right)
$$

for some positive constants $K_{1}$ and $K_{2}$ that are independent of $n$.

(Z2) One has

$$
\alpha \leq w \quad ; \quad-\frac{1}{\alpha} \leq \gamma \leq \frac{1}{w-\alpha} \quad ; \quad 0<K \leq\left(\frac{c^{\alpha}}{2 K_{2}}\right)^{\frac{1}{w-\alpha}}
$$

Notice that (Z2) can always be forced by increasing $w$, and then decreasing $K$ and $\gamma$ in the bounds of Condition $(\mathrm{Z} 1)$. If Conditions $(\mathrm{Z} 1)$ and $(\mathrm{Z} 2)$ are satisfied, then we say that we have a zone of control $\left[-K\left(t_{n}\right)^{\gamma}, K\left(t_{n}\right)^{\gamma}\right]$ with index $(v, w)$.

Note that although the definition of zone of control depends on the reference law $\phi_{c, \alpha, \beta}$, the latter does not appear in the terminology (throughout the paper, it is considered fixed).

Proposition 6. Let $\left(X_{n}\right)_{n \in \mathbb{N}}$ be a sequence of random variables, $\phi_{c, \alpha, \beta}$ a reference stable law, $Y$ with distribution $\phi_{c, \alpha, \beta}$ and $Y_{n}$ as in Proposition 3. Assume that $\left(X_{n}\right)_{n \in \mathbb{N}}$ has a zone of control $\left[-K\left(t_{n}\right)^{\gamma}, K\left(t_{n}\right)^{\gamma}\right]$ with index $(v, w)$. If $\gamma>-\frac{1}{\alpha}$, then one has the convergence in law $Y_{n} \rightarrow Y$.

Proof. Condition (Z1) implies that, if $Y_{n}$ is the renormalization of $X_{n}$ and $Y \sim \phi_{c, \alpha, \beta}$, then for fixed $\xi$,

$$
\left|\frac{\mathbb{E}\left[\mathrm{e}^{\mathrm{i} \xi Y_{n}}\right]}{\mathbb{E}\left[\mathrm{e}^{\mathrm{i} \xi Y}\right]}-1\right|=\left|\theta_{n}\left(\frac{\xi}{\left(t_{n}\right)^{1 / \alpha}}\right)-1\right| \leq \frac{K_{1}|\xi|^{v}}{\left(t_{n}\right)^{v / \alpha}} \exp \left(\frac{K_{2}|\xi|^{w}}{\left(t_{n}\right)^{w / \alpha}}\right)
$$

for $t_{n}$ large enough, and the right-hand side goes to 0 . This proves the convergence in law $Y_{n} \rightarrow Y$.

The goal of the next few sections will be to get some speed of convergence estimates for this convergence in distribution.

Remark 7. In the definition of zone of control, we do not assume the mod- $\phi_{c, \alpha, \beta}$ convergence of the sequence $\left(X_{n}\right)_{n \in \mathbb{N}}$ with parameters $\left(t_{n}\right)_{n \in \mathbb{N}}$ and $\operatorname{limit}_{\lim _{n \rightarrow \infty}} \theta_{n}(\xi)=\theta(\xi)$. However, in almost all the examples considered, we shall indeed have (complex) mod- $\phi$ convergence (convergence of the residues $\theta_{n}$ ), with the same parameters $t_{n}$ as for the notion of zone of control. We shall then speak of mod- $\phi$ convergence with a zone of convergence $\left[-K\left(t_{n}\right)^{\gamma}, K\left(t_{n}\right)^{\gamma}\right]$ and with index of control $(v, w)$. Mod- $\phi$ convergence implies other probabilistic results than estimates of Berry-Esseen type: central limit theorem with a large range of normality, moderate deviations ( $c f$. [FMN16]), local limit theorem ([DKN15]), etc.

Remark 8. If one has mod- $\phi_{c, \alpha, \beta}$ convergence of $\left(X_{n}\right)_{n \in \mathbb{N}}$, then there is at least a zone of convergence $[-K, K]$ of index $(v, w)=(0,0)$, with $\gamma=0$; indeed, the residues $\theta_{n}(\xi)$ stay locally bounded under this hypothesis. Thus, Definition 5 is an extension of this statement. However, we allow in the definition the exponent $\gamma$ to be negative (but not smaller than $-\frac{1}{\alpha}$ ). Indeed, in the computation of Berry-Esseen type bounds, we shall sometimes need to work with smaller zones than the one given by mod- $\phi$ convergence, see the hypotheses of Theorem 20, and Sections 3.3 and 3.4 for examples. 
Remark 9. In our definition of zone of control, we ask for a bound on $\left|\theta_{n}(\xi)-1\right|$ that holds for any $n \in \mathbb{N}$. Of course, if the bound is only valid for $n \geq n_{0}$ large enough, then the corresponding bound on the Kolmogorov distance (Theorem 20) will only hold for $n \geq n_{0}$.

\subsection{Spaces of test functions}

Until the end of Section 2, all the spaces of functions considered will be spaces of complex valued functions on the real line. If $f \in \mathrm{L}^{1}(\mathbb{R})$, we denote its Fourier transform

$$
\widehat{f}(\xi)=\int_{\mathbb{R}} \mathrm{e}^{\mathrm{i} x \xi} f(x) d x .
$$

Recall that the Schwartz space $\mathscr{S}(\mathbb{R})$ is by definition the space of infinitely differentiable functions whose derivatives tend to 0 at infinity faster than any power of $x$. Restricted to $\mathscr{S}(\mathbb{R})$, the Fourier transform is an automorphism, and it satisfies the Parseval formula

$$
\forall f, g \in \mathscr{S}(\mathbb{R}), \int_{\mathbb{R}} f(x) \overline{g(x)} d x=\frac{1}{2 \pi} \int_{\mathbb{R}} \widehat{f}(\xi) \overline{\widehat{g}(\xi)} d \xi .
$$

We refer to [Lan93, Chapter VIII] and [Rud91, Part II] for a proof of this formula, and for the theory of Fourier transforms. The Parseval formula allows to extend by duality and/or density the Fourier transform to other spaces of functions or distributions. In particular, if $f \in \mathrm{L}^{2}(\mathbb{R})$, then its Fourier transform $\widehat{f}$ is well defined in $L^{2}(\mathbb{R})$, although in general the integral $\int_{\mathbb{R}} \mathrm{e}^{\mathrm{i} x \xi} f(x) d x$ does not converge; and we have again the Parseval formula

$$
\forall f, g \in \mathrm{L}^{2}(\mathbb{R}), \int_{\mathbb{R}} f(x) \overline{g(x)} d x=\frac{1}{2 \pi} \int_{\mathbb{R}} \widehat{f}(\xi) \overline{\widehat{g}(\xi)} d \xi,
$$

which amounts to the fact that $f \mapsto \frac{1}{\sqrt{2 \pi}} \widehat{f}$ is an isometry of $\mathrm{L}^{2}(\mathbb{R})$ (see [Rud91, §7.9]).

We denote $\mathscr{M}^{1}(\mathbb{R})$ the set of probability measures on Borel subsets of $\mathbb{R}$. In the sequel, we will need to apply a variant of Parseval's formula, where $\overline{g(x)} d x$ is replaced by $\mu(d x)$, with $\mu$ in $\mathscr{M}^{1}(\mathbb{R})$. This is given in the following lemma (see [Str11, Lemma 2.3.3], or [Mal95, p. 134]).

Lemma 10. For any function $f \in \mathrm{L}^{1}(\mathbb{R})$ with $\widehat{f} \in \mathrm{L}^{1}(\mathbb{R})$, and any Borel probability measure $\mu \in \mathscr{M}^{1}(\mathbb{R})$, the pairing $\langle\mu \mid f\rangle=\int_{\mathbb{R}} f(x) \mu(d x)$ is well defined, and the Parseval formula holds:

$$
\int_{\mathbb{R}} f(x) \mu(d x)=\frac{1}{2 \pi} \int_{\mathbb{R}} \widehat{f}(\xi) \widehat{\mu}(-\xi) d \xi,
$$

where $\widehat{\mu}(\xi)=\int_{\mathbb{R}} \mathrm{e}^{\mathrm{i} \xi x} \mu(d x)$. The formula also holds for finite signed measures.

Let us now introduce two adequate spaces of test functions, for which we shall be able to prove speed of convergence estimates. We first consider functions $f \in \mathrm{L}^{1}(\mathbb{R})$ with compactly supported Fourier transforms:

Definition 11. We call smooth test function of order 0 , or simply smooth test function an element $f \in \mathrm{L}^{1}(\mathbb{R})$ whose Fourier transform is compactly supported. We denote $\mathscr{T}_{0}(\mathbb{R})$ the subspace of $\mathrm{L}^{1}(\mathbb{R})$ that consists in smooth test functions; it is an ideal for the convolution product.

Example 12. If

$$
\operatorname{sinc}(x):=\frac{\sin x}{x}=\frac{1}{2} \int_{-1}^{1} \mathrm{e}^{\mathrm{i} x \xi} d \xi
$$

then by Fourier inversion $\widehat{\operatorname{sinc}}(\xi)=\pi 1_{|\xi| \leq 1}$ is compactly supported on $[-1,1]$. Therefore, $f(x)=$ $(\operatorname{sinc}(x))^{2}$ is an element of $\mathrm{L}^{1}(\mathbb{R})$ whose Fourier transform is compactly supported on $[-1,1]+[-1,1]=$ $[-2,2]$, and $f \in \mathscr{T}_{0}(\mathbb{R})$. 
Let us comment a bit Definition 11. If $f$ is in $\mathscr{T}_{0}(\mathbb{R})$, then its Fourier transform $\widehat{f}$ is bounded by $\|f\|_{\mathrm{L}^{1}}$ and vanishes outside an interval $[-C, C]$, so $\widehat{f} \in \mathrm{L}^{1}(\mathbb{R})$. Since $f$ and $\widehat{f}$ are integrable, we can apply Lemma 10 with $f$. Moreover, $f$ is then known to satisfy the Fourier inversion formula (see [Rud91, §7.7]):

$$
f(x)=\frac{1}{2 \pi} \int_{\mathbb{R}} \widehat{f}(\xi) \mathrm{e}^{-\mathrm{i} \xi x} d \xi
$$

As the integral above is in fact on a compact interval $[-C, C]$, the standard convergence theorems ensure that $f$ is infinitely differentiable in $x$, hence the term "smooth". Also, by applying the Riemann-Lebesgue lemma to the continuous compactly supported functions $\xi \mapsto(-\mathrm{i} \xi)^{k} \hat{f}(\xi)$, one sees that $f(x)$ and all its derivatives $f^{(k)}(x)$ go to 0 as $x$ goes to infinity. To conclude, $\mathscr{T}_{0}(\mathbb{R})$ is included in the space $\mathscr{C}_{0}^{\infty}(\mathbb{R})$ of smooth functions whose derivatives all vanish at infinity.

Actually, we will need to work with more general test functions, defined by using the theory of tempered distributions. We endow the Schwartz space $\mathscr{S}(\mathbb{R})$ of smooth rapidly decreasing functions with its usual topology of metrizable locally convex topological vector space, defined by the family of semi-norms

$$
\|f\|_{k, l}=\sum_{a \leq k} \sum_{b \leq l} \sup _{x \in \mathbb{R}}\left|x^{a}\left(\partial^{b} f\right)(x)\right| .
$$

We recall that a tempered distribution $\psi$ is a continuous linear form $\psi: \mathscr{S}(\mathbb{R}) \rightarrow \mathbb{C}$. The value of a tempered distribution $\psi$ on a smooth function $f$ will be denoted $\psi(f)$ or $\langle\psi \mid f\rangle$. The space of all tempered distributions is classically denoted $\mathscr{S}^{\prime}(\mathbb{R})$, and it is endowed with the $*$-weak topology. The spaces of integrable functions, of square integrable functions and of probability measures can all be embedded in the space $\mathscr{S}^{\prime}(\mathbb{R})$ as follows: if $f$ is a function in $L^{1}(\mathbb{R}) \cup L^{2}(\mathbb{R})$, or if $\mu$ is in $\mathscr{M}^{1}(\mathbb{R})$, then we associate to them the distributions

$$
\langle f \mid g\rangle=\int_{\mathbb{R}} f(x) g(x) d x \quad ; \quad\langle\mu \mid g\rangle=\int_{\mathbb{R}} g(x) \mu(d x) .
$$

We then say that these distributions are represented by the function $f$ and by the measure $\mu$.

The Fourier transform of a tempered distribution $\psi$ is defined by duality: it is the unique tempered distribution $\widehat{\psi}$ such that

$$
\langle\widehat{\psi} \mid f\rangle=\langle\psi \mid \widehat{f}\rangle
$$

for any $f \in \mathscr{S}(\mathbb{R})$. This definition agrees with the previous definitions of Fourier transforms for integrable functions, square integrable functions, or probability measures (all these elements can be paired with Schwartz functions). Similarly, if $\psi$ is a tempered distribution, then one can also define by duality its derivative: thus, $\partial \psi$ is the unique tempered distribution such that

$$
\langle\partial \psi \mid f\rangle=-\langle\psi \mid \partial f\rangle
$$

for any $f \in \mathscr{S}(\mathbb{R})$. The definition agrees with the usual one when $\psi$ comes from a derivable function, by the integration by parts formula. On the other hand, Fourier transform and derivation define linear endomorphisms of $\mathscr{S}^{\prime}(\mathbb{R})$; also note that the Fourier transform is bijective.

Definition 13. A smooth test function of order 1 , or smooth test distribution is a distribution $f \in$ $\mathscr{S}^{\prime}(\mathbb{R})$, such that $\partial f$ is in $\mathscr{T}_{0}(\mathbb{R})$, that is to say that the distribution $\partial f$ can be represented by an integrable function with compactly supported Fourier transform. We denote $\mathscr{T}_{1}(\mathbb{R})$ the space of smooth test distributions.

We now discuss Parseval's formula for functions in $\mathscr{T}_{1}(\mathbb{R})$.

Proposition 14. Any smooth test distribution $f \in \mathscr{T}_{1}(\mathbb{R})$ can be represented by a bounded function in $\mathscr{C}^{\infty}(\mathbb{R})$. Moreover, for any smooth test distribution in $\mathscr{T}_{1}(\mathbb{R})$ :

(TD1) If $\mu$ is a Borel probability measure, then the pairing $\langle\mu \mid f\rangle=\int_{\mathbb{R}} f(x) \mu(d x)$ is well defined. 


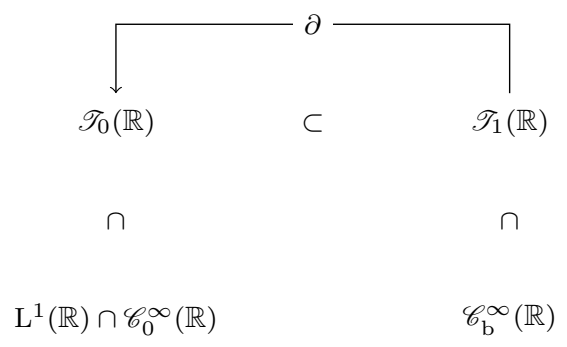

Fig. 2 The two spaces of test functions $\mathscr{T}_{0}(\mathbb{R})$ and $\mathscr{T}_{1}(\mathbb{R})$.

(TD2) The tempered distribution $\widehat{f}$ can be paired with the Fourier transform $\widehat{\mu}$ of a probability measure with finite first moment, in a way that extends the pairing between $\mathscr{S}^{\prime}(\mathbb{R})$ and $\mathscr{S}(\mathbb{R})$ when $\mu$ (and therefore $\widehat{\mu})$ is given by a Schwartz density.

(TD3) The Parseval formula holds: if $f \in \mathscr{T}_{1}(\mathbb{R})$ and $\mu$ has finite expectation, then

$$
\langle\mu \mid f\rangle=\frac{1}{2 \pi}\langle\widehat{f} \mid \widehat{\mu}\rangle .
$$

Proof. We start by giving a better description of the tempered distributions $f$ and $\widehat{f}$. Denote $\phi=\partial f$; by assumption, this tempered distribution can be represented by a function $\phi \in \mathscr{T}_{0}(\mathbb{R})$, which in particular is of class $\mathscr{C}^{\infty}$ and integrable. Set

$$
\widetilde{f}(x)=\int_{y=0}^{x} \phi(y) d y .
$$

This is a function of class $\mathscr{C}^{\infty}$, whose derivative is $\phi$, and which is bounded since $\phi$ is integrable. Therefore, it is a tempered distribution, and for any $g \in \mathscr{S}(\mathbb{R})$,

$$
\langle\partial f \mid g\rangle=\langle\phi \mid g\rangle=\langle\partial \tilde{f} \mid g\rangle .
$$

We conclude that $\partial(f-\tilde{f})$ is the zero distribution. It is then a standard result that, given a tempered distribution $\psi$, one has $\partial \psi=0$ if and only if $\psi$ can be represented a constant. So,

$$
f(x)=\int_{y=0}^{x} \phi(y) d y+f(0) .
$$

This shows in particular that $f$ is a smooth bounded function.

A similar description can be provided for $\widehat{f}$. Recall that the principal value distribution, denoted $\operatorname{pv}\left(\frac{1}{x}\right)$, is the tempered distribution defined for any $g \in \mathscr{S}(\mathbb{R})$ by

$$
\left\langle\operatorname{pv}\left(\frac{1}{x}\right) \mid g\right\rangle=\lim _{\varepsilon \rightarrow 0}\left(\int_{|x| \geq \varepsilon} \frac{g(x)}{x} d x\right) .
$$

The existence of the limit is easily proved by making a Taylor expansion of $g$ around 0 . Denote

$$
\begin{aligned}
\mathscr{S}^{[1]} & =\{g \in \mathscr{S}(\mathbb{R}) \mid g(x)=x h(x) \text { with } h \in \mathscr{S}(\mathbb{R})\} ; \\
\mathscr{S}_{[1]} & =\{g \in \mathscr{S}(\mathbb{R}) \mid g=\partial h \text { with } h \in \mathscr{S}(\mathbb{R})\} ;
\end{aligned}
$$

the Fourier transform establishes an homeomorphism between $\mathscr{S}^{[1]}$ and $\mathscr{S}_{[1]}$, and the restriction of $\mathrm{pv}\left(\frac{1}{x}\right)$ to $\mathscr{S}^{[1]}$ is

$$
\left\langle\operatorname{pv}\left(\frac{1}{x}\right) \mid g\right\rangle=\int_{\mathbb{R}} \frac{g(x)}{x} d x .
$$


Let $\widehat{g}(\xi)$ be an element of $\mathscr{S}^{[1]}$, which we write as $\widehat{g}(\xi)=(-\mathrm{i} \xi) \widehat{h}(\xi)$ for some $h \in \mathscr{S}(\mathbb{R})$. This is equivalent to $g(x)=(\partial h)(x)$. Let us denote $g_{-}(x)=g(-x), h_{-}(x)=h(-x)$, and $\mathrm{pv}\left(\frac{\mathrm{i} \widehat{\phi}(\xi)}{\xi}\right)$ the tempered distribution defined by

$$
\operatorname{pv}\left(\frac{\mathrm{i} \widehat{\phi}(\xi)}{\xi}\right)=\operatorname{ipv}\left(\frac{1}{\xi}\right) \circ m_{\widehat{\phi}},
$$

with $m_{\widehat{\phi}}: \mathscr{S}(\mathbb{R}) \rightarrow \mathscr{S}(\mathbb{R})$ equal to the multiplication by $\widehat{\phi}$. Then we can make the following computation:

$$
\begin{aligned}
\langle\hat{f} \mid \widehat{g}\rangle & =\langle f \mid \widehat{\widehat{g}}\rangle=2 \pi\left\langle f \mid g_{-}\right\rangle=-2 \pi\left\langle f \mid \partial h_{-}\right\rangle=2 \pi\left\langle\phi \mid h_{-}\right\rangle=\langle\phi \mid \widehat{\hat{h}}\rangle \\
& =\langle\widehat{\phi} \mid \widehat{h}\rangle=\left\langle\widehat{\phi} \mid \frac{\mathrm{i} \widehat{g}(\xi)}{\xi}\right\rangle=\left\langle\operatorname{pv}\left(\frac{\mathrm{i} \widehat{\phi}(\xi)}{\xi}\right) \mid \widehat{g}\right\rangle .
\end{aligned}
$$

Thus, the tempered distributions $\widehat{f}$ and $\operatorname{pv}\left(\frac{\mathrm{i} \widehat{\phi}(\xi)}{\xi}\right)$ agree on the codimension 1 subspace $\mathscr{S}^{[1]}$ of $\mathscr{S}(\mathbb{R})$. However, $\mathscr{S}^{[1]}$ is also the space of functions in $\mathscr{S}(\mathbb{R})$ that vanish at 0 , so, if $g_{0}$ is any function in $\mathscr{S}(\mathbb{R})$ such that $g_{0}(0)=1$, then for $g \in \mathscr{S}(\mathbb{R})$,

$$
\begin{aligned}
\langle\widehat{f} \mid g\rangle & =\left\langle\widehat{f} \mid g-g(0) g_{0}\right\rangle+g(0)\left\langle\widehat{f} \mid g_{0}\right\rangle \\
& =\left\langle\operatorname{pv}\left(\frac{\mathrm{i} \widehat{\phi}(\xi)}{\xi}\right) \mid g-g(0) g_{0}\right\rangle+\left\langle\widehat{f} \mid g_{0}\right\rangle\left\langle\delta_{0} \mid g\right\rangle \\
& =\left\langle\operatorname{pv}\left(\frac{\mathrm{i} \widehat{\phi}(\xi)}{\xi}\right) \mid g\right\rangle+\left(\left\langle\widehat{f}-\mathrm{pv}\left(\frac{\mathrm{i} \hat{\phi}(\xi)}{\xi}\right) \mid g_{0}\right\rangle\right)\left\langle\delta_{0} \mid g\right\rangle \\
& =\left\langle\operatorname{pv}\left(\frac{\mathrm{i} \widehat{\phi}(\xi)}{\xi}\right)+L \delta_{0} \mid g\right\rangle
\end{aligned}
$$

where $L$ is some constant. Thus,

$$
\widehat{f}(\xi)=\operatorname{pv}\left(\frac{\mathrm{i} \widehat{\phi}(\xi)}{\xi}\right)+L \delta_{0}
$$

and a computation against test functions shows that

$$
L=2 \pi f(0)-\mathrm{i}\left\langle\operatorname{pv}\left(\frac{1}{\xi}\right) \mid \widehat{\phi}\right\rangle .
$$

The three parts of the proposition are now easily proven. For (TD1), since $f(x)$ is smooth and bounded, we can indeed consider the convergent integral $\int_{\mathbb{R}} f(x) \mu(d x)$. For (TD2), assuming that $\mu$ has a finite first moment, $\widehat{\mu}$ is a function of class $\mathscr{C}^{1}$ and with bounded derivative. The same holds for $\widehat{\phi} \widehat{\mu}$, and therefore, one can define

$$
\begin{aligned}
\int_{\mathbb{R}} \widehat{f}(\xi) \widehat{\mu}(-\xi) d \xi & =\mathrm{i}\left\langle\operatorname{pv}\left(\frac{1}{\xi}\right) \mid \widehat{\phi} \widehat{\widehat{\mu}}\right\rangle+L \\
& =\left(\lim _{\varepsilon \rightarrow \infty} \int_{|x| \geq \varepsilon} \frac{\mathrm{i} \widehat{\phi}(\xi) \widehat{\mu}(-\xi)}{\xi} d \xi\right)+L
\end{aligned}
$$

Indeed, if $f \in \mathscr{C}^{1}(\mathbb{R})$, then $\lim _{\varepsilon \rightarrow \infty} \int_{1 \geq|x| \geq \varepsilon} \frac{f(x)}{x} d x$ always exists, as can be seen by replacing $f$ by its Taylor approximation at 0 . Finally, let us prove the Parseval formula (TD3). The previous calculations show that 


$$
\begin{aligned}
\frac{1}{2 \pi} \int_{\mathbb{R}} \widehat{f}(\xi) \widehat{\mu}(-\xi) d \xi & =\frac{\mathrm{i}}{2 \pi}\left\langle\operatorname{pv}\left(\frac{1}{\xi}\right) \mid \widehat{\phi} \bar{\mu}-\widehat{\phi}\right\rangle+f(0) \\
& =\lim _{\varepsilon \rightarrow 0}\left(\frac{\mathrm{i}}{2 \pi} \int_{|\xi| \geq \varepsilon} \widehat{\phi}(\xi)\left(\frac{\widehat{\mu}(-\xi)-1}{\xi}\right) d \xi\right)+f(0) \\
& =\frac{1}{2 \pi} \int_{\mathbb{R}} \widehat{\phi}(\xi)\left(\frac{\widehat{\mu}(-\xi)-1}{-\mathrm{i} \xi}\right) d \xi+f(0) .
\end{aligned}
$$

Indeed, the function $\xi \mapsto \frac{\widehat{\mu}(-\xi)-1}{-\mathrm{i} \xi}$ is continuous on $\mathbb{R}$ and bounded, with value $\frac{\widehat{\mu}^{\prime}(0)}{\mathrm{i}}=\int_{\mathbb{R}} x \mu(d x)$ at $\xi=0$; it can therefore be integrated against the function $\widehat{\phi}$ which is integrable (and even with compact support). On the other hand,

$$
\begin{aligned}
\int_{\mathbb{R}} f(x) \mu(d x) & =\int_{x \in \mathbb{R}} \int_{y=0}^{x} \phi(y) d y \mu(d x)+f(0) \\
& =\int_{(x, y) \in \mathbb{R}^{2}}\left(1_{x>y>0}-1_{x<y<0}\right) \phi(y) d y \mu(d x)+f(0) \\
& =\int_{y \in \mathbb{R}} \phi(y) F(y) d y+f(0), \quad \text { with } F(y)=\mu((y, \infty))-1_{y \leq 0} .
\end{aligned}
$$

One has $\int_{\mathbb{R}}|F(y)| d y=\int_{y=0}^{\infty} \mu((y, \infty))+\int_{y=-\infty}^{0} \mu((-\infty, y))=\int_{\mathbb{R}}|x| \mu(d x)$, which is finite. In the integral above, we can therefore consider $F(y) d y$ as a finite signed measure, and the Parseval formula applies by Lemma 10. One computes readily

$$
\widehat{F}(\xi)=\frac{\widehat{\mu}(\xi)-1}{\mathrm{i} \xi},
$$

which ends the proof.

Remark 15. The Parseval formula of Proposition 14 extends readily to finite signed measures $\mu$ such that $\int_{\mathbb{R}}|x||\mu|(d x)<+\infty$. Actually, it is sufficient to have a finite signed measure $\mu$ such that

$$
\frac{\widehat{\mu}(\xi)-\widehat{\mu}(0)}{\xi^{v}}
$$

is bounded in a vicinity of 0 , for some $v>0$. Then, $(\widehat{\mu}(\xi)-\widehat{\mu}(0)) / \xi$ is integrable in a neighborhood of 0 . This ensures that the distribution $f(x)$ (respectively, the distribution $\widehat{f}(\xi)$ ) can be evaluated against the measure $\mu(x)$ (respectively, against $\widehat{\mu}(\xi)$ ), and then, the proof of Parseval's formula is analogous to the previous arguments.

\subsection{Estimates for test functions}

We now give an estimate of $\mathbb{E}\left[f_{n}\left(Y_{n}\right)\right]-\mathbb{E}\left[f_{n}(Y)\right]$, where $\left(f_{n}\right)_{n \in \mathbb{N}}$ is a sequence of test functions in $\mathscr{T}_{0}(\mathbb{R})$ or $\mathscr{T}_{1}(\mathbb{R})$, and $\left(Y_{n}\right)_{n \in \mathbb{N}}$ is a sequence of random variables associated to a sequence $\left(X_{n}\right)_{n \in \mathbb{N}}$ which has a zone of control.

Proposition 16. Let $\left(X_{n}\right)_{n \in \mathbb{N}}$ be a sequence of random variables, $\phi_{c, \alpha, \beta}$ a reference stable law, $Y$ with law $\phi_{c, \alpha, \beta}$ and $Y_{n}$ as in Proposition 3. We assume that:

(1) $\left(X_{n}\right)_{n \in \mathbb{N}}$ has a zone of control $\left[-K\left(t_{n}\right)^{\gamma}, K\left(t_{n}\right)^{\gamma}\right]$ with index $(v, w)$;

(2) $\left(f_{n}\right)_{n \in \mathbb{N}}$ is a sequence of smooth test functions in $\mathscr{T}_{0}(\mathbb{R})$, such that the support of $\widehat{f}_{n}$ is included into $\left[-K\left(t_{n}\right)^{\gamma+1 / \alpha}, K\left(t_{n}\right)^{\gamma+1 / \alpha}\right]$.

Then, 


$$
\left|\mathbb{E}\left[f_{n}\left(Y_{n}\right)\right]-\mathbb{E}\left[f_{n}(Y)\right]\right| \leq C_{0}(c, \alpha, v) K_{1} \frac{\left\|f_{n}\right\|_{\mathrm{L}^{1}}}{\left(t_{n}\right)^{v / \alpha}}
$$

where $C_{0}(c, \alpha, v)=\frac{2^{\frac{v+1}{\alpha}} \Gamma((v+1) / \alpha)}{\pi \alpha c^{v+1}}$.

If instead of (2) we assume:

(2') $\left(f_{n}\right)_{n \in \mathbb{N}}$ is a sequence of smooth test distributions in $\mathscr{T}_{1}(\mathbb{R})$ such that if $\phi_{n}=\partial f_{n}$ is the derivative of the distribution $f_{n}$, then the support of $\widehat{\phi}_{n}$ is included into $\left[-K\left(t_{n}\right)^{\gamma+1 / \alpha}, K\left(t_{n}\right)^{\gamma+1 / \alpha}\right]$.

Then

$$
\left|\mathbb{E}\left[f_{n}\left(Y_{n}\right)\right]-\mathbb{E}\left[f_{n}(Y)\right]\right| \leq C_{1}(c, \alpha, v) K_{1} \frac{\left\|\phi_{n}\right\|_{\mathrm{L}^{1}}}{\left(t_{n}\right)^{v / \alpha}}
$$

where $C_{1}(c, \alpha, v)=\frac{2^{v / \alpha} \Gamma(v / \alpha)}{\pi \alpha c^{v}}$.

Proof. Consider first a sequence $\left(f_{n}\right)_{n \in \mathbb{N}}$ of test functions in $\mathscr{T}_{0}(\mathbb{R})$, which satisfies (2). Using Parseval formula and the zone of control assumption, we have

$$
\begin{aligned}
& \mathbb{E}\left[f_{n}\left(Y_{n}\right)\right]-\mathbb{E}\left[f_{n}(Y)\right]=\frac{1}{2 \pi} \int_{-K\left(t_{n}\right)^{\gamma+\frac{1}{\alpha}}}^{K\left(t_{n}\right)^{\gamma+\frac{1}{\alpha}}} \widehat{f}_{n}(\xi) \mathrm{e}^{\eta(-\mathrm{i} \xi)}\left(\theta_{n}\left(-\xi /\left(t_{n}\right)^{\frac{1}{\alpha}}\right)-1\right) d \xi \\
&\left|\mathbb{E}\left[f_{n}\left(Y_{n}\right)\right]-\mathbb{E}\left[f_{n}(Y)\right]\right| \leq \frac{K_{1}\left\|\widehat{f}_{n}\right\|_{\infty}}{2 \pi\left(t_{n}\right)^{v / \alpha}} \int_{-K\left(t_{n}\right)^{\gamma+\frac{1}{\alpha}}}^{K\left(t_{n}\right)^{\gamma+\frac{1}{\alpha}}}|\xi|^{v} \mathrm{e}^{-|c \xi|^{\alpha}+K_{2}\left(\frac{|\xi|}{\left(t_{n}\right)^{1 / \alpha}}\right)^{w}} d \xi
\end{aligned}
$$

For $\xi$ in $\left[-K\left(t_{n}\right)^{\gamma+1 / \alpha}, K\left(t_{n}\right)^{\gamma+1 / \alpha}\right]$, since $\left(t_{n}\right)^{\gamma-1 /(w-\alpha)} \leq 1$, the second term in the exponent can be bounded as follows:

$$
\begin{aligned}
K_{2}\left(\frac{|\xi|}{\left(t_{n}\right)^{1 / \alpha}}\right)^{w}=K_{2}|\xi|^{\alpha}\left(\frac{|\xi|}{\left(t_{n}\right)^{\frac{1}{\alpha}+\frac{1}{w-\alpha}}}\right)^{w-\alpha} & \leq K_{2}|\xi|^{\alpha}\left(K\left(t_{n}\right)^{\gamma-\frac{1}{w-\alpha}}\right)^{w-\alpha} \\
& \leq \frac{|c \xi|^{\alpha}}{2}
\end{aligned}
$$

This is compensated by the term $-|c \xi|^{\alpha}$ and, therefore,

$$
\begin{aligned}
\left|\mathbb{E}\left[f_{n}\left(Y_{n}\right)\right]-\mathbb{E}\left[f_{n}(Y)\right]\right| & \leq \frac{K_{1}\left\|\widehat{f}_{n}\right\|_{\infty}}{2 \pi\left(t_{n}\right)^{v / \alpha}} \int_{\mathbb{R}}|\xi|^{v} \mathrm{e}^{-\frac{|c \xi|^{\alpha}}{2}} d \xi \\
& \leq \frac{2^{\frac{v+1}{\alpha}} K_{1}}{\pi \alpha c^{v+1}\left(t_{n}\right)^{v / \alpha}} \Gamma\left(\frac{v+1}{\alpha}\right)\left\|f_{n}\right\|_{\mathrm{L}^{1}} .
\end{aligned}
$$

This ends the proof of the first case. For test distributions $f_{n} \in \mathscr{T}_{1}(\mathbb{R})$ which satisfies the condition $\left(2^{\prime}\right)$, let us introduce the signed measure $\mu=\mathbb{P}_{Y_{n}}-\mathbb{P}_{Y}$. One has $\widehat{\mu}(0)=0$, and by hypothesis,

$$
\left|\frac{\widehat{\mu}(\xi)}{\xi}\right| \leq \frac{K_{1}|\xi|^{v-1}}{\left(t_{n}\right)^{v / \alpha}} \mathrm{e}^{-|c \xi|^{\alpha}+K_{2}\left(\frac{|\xi|}{\left(t_{n}\right)^{1 / \alpha}}\right)^{w}} .
$$

Remark 15 applies, and thus,

$$
\left|\mathbb{E}\left[f_{n}\left(Y_{n}\right)\right]-\mathbb{E}\left[f_{n}(Y)\right]\right|=\left|\left\langle\mu \mid f_{n}\right\rangle\right|=\frac{1}{2 \pi}\left|\left\langle\widehat{f_{n}} \mid \overline{\widehat{\mu}}\right\rangle\right|=\frac{1}{2 \pi}\left|\int_{\mathbb{R}} \widehat{\phi_{n}}(\xi) \frac{\widehat{\mu}(-\xi)}{\xi} d \xi\right| .
$$

From there, the computations are exacly the same as before, with an index $v-1$ instead of $v$. 


\subsection{Smoothing techniques}

We now explain how to relate the estimates on test functions or distributions to estimates on the Kolmogorov distance. The main tool with respect to this problem is the following:

Lemma 17. There exists a function $\rho$ (called kernel) on $\mathbb{R}$ with the following properties.

1. The kernel $\rho$ is non-negative, with $\int_{\mathbb{R}} \rho(x) d x=1$.

2. The support of $\hat{\rho}$ is $[-1,1]$ (hence, $\rho$ is a test function in $\mathscr{T}_{0}(\mathbb{R})$ ).

3. The functions $\rho$ and $\hat{\rho}$ are even, and

$$
\rho(K) \leq \min \left(\frac{3}{8 \pi}, \frac{96}{\pi K^{4}}\right) .
$$
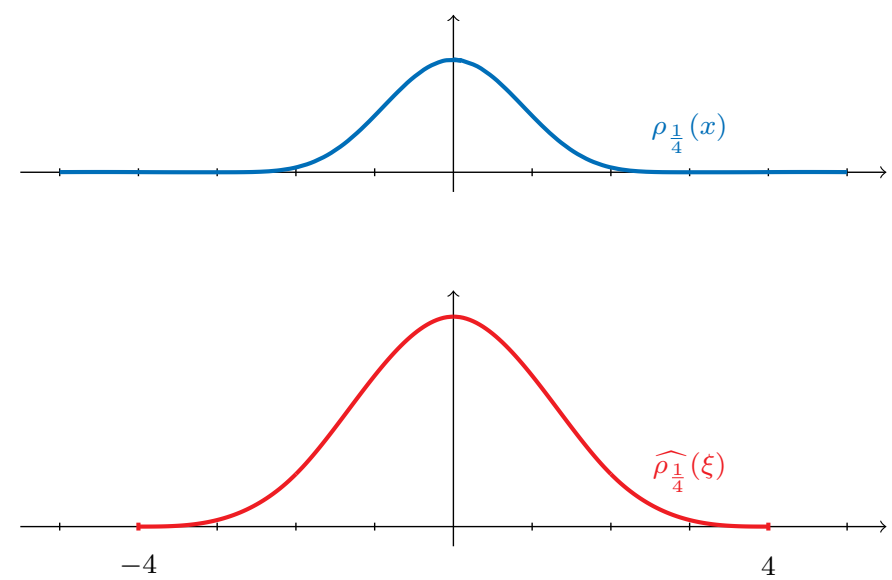

Fig. 3 The smoothing kernel $\rho_{\frac{1}{4}}$, and its Fourier transform which is supported on $[-4,4]$.

Proof. Set

$$
\rho(x)=\frac{3}{8 \pi}\left(\operatorname{sinc}\left(\frac{x}{4}\right)\right)^{4} .
$$

It has its Fourier transform supported on $\left[-\frac{1}{4}, \frac{1}{4}\right]+\left[-\frac{1}{4}, \frac{1}{4}\right]+\left[-\frac{1}{4}, \frac{1}{4}\right]+\left[-\frac{1}{4}, \frac{1}{4}\right]=[-1,1]$. On the other hand, an easy computation gives $\int_{\mathbb{R}} \rho(x) d x=1$ : use for example the Plancherel formula

$$
\int_{\mathbb{R}}|f(x)|^{2} d x=\frac{1}{2 \pi} \int_{\mathbb{R}}|\widehat{f}(\xi)|^{2} d \xi
$$

with $f(x)=\operatorname{sinc}(x)^{2}$ and thus $\widehat{f}(\xi)=\frac{1}{2 \pi} \widehat{\operatorname{sinc}} * \widehat{\operatorname{sinc}}(\xi)=\frac{\pi}{2}(2-|\xi|)_{+}$. Finally, $\operatorname{sinc}(x) \leq \min \left(1, \frac{1}{|x|}\right)$, which leads to the inequality stated for $\rho(K)$.

In the following, for $\varepsilon>0$, we set $\rho_{\varepsilon}(x)=\frac{1}{\varepsilon} \rho\left(\frac{x}{\varepsilon}\right)$, which has its Fourier transform compactly supported on $\left[-\frac{1}{\varepsilon}, \frac{1}{\varepsilon}\right]$; see Figure 3. We also denote $f_{a, \varepsilon}(x)=f_{\varepsilon}(x-a)$, where $f_{\varepsilon}$ is the function $1_{(-\infty, 0]} * \rho_{\varepsilon} ; c f$. Figure 4. For all $a, \varepsilon, f_{a, \varepsilon}$ is an approximation of the Heaviside function $1_{(-\infty, a]}$, and one has the following properties:

Proposition 18. The function $f_{a, \varepsilon}$ is a smooth test distribution in $\mathscr{T}_{1}(\mathbb{R})$ whose derivative $\partial f_{a, \varepsilon}$ has its Fourier transform compactly supported on $\left[-\frac{1}{\varepsilon}, \frac{1}{\varepsilon}\right]$, and satisfies $\left\|\partial f_{a, \varepsilon}\right\|_{\mathrm{L}^{1}}=1$. Moreover: 


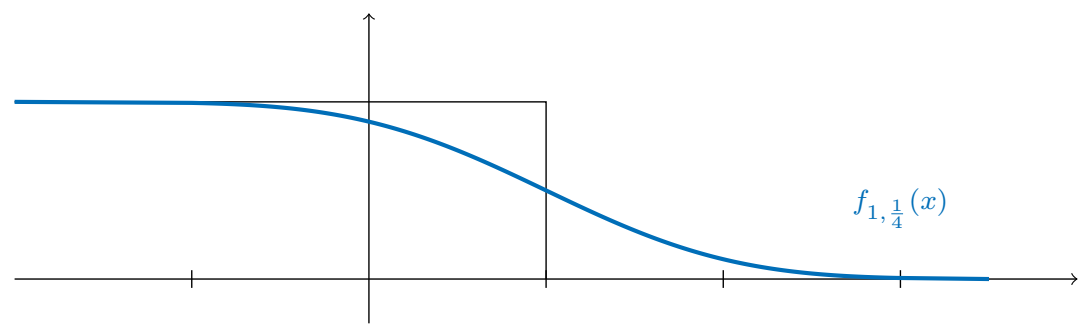

Fig. 4 The approximation $f_{1, \frac{1}{4}}$ of the Heaviside function $1_{(-\infty, 1]}$.

1. The function $f_{a, \varepsilon}$ has a non-positive derivative, and decreases from 1 to 0.

2. One has $f_{1}(x)=1-f_{1}(-x)$, and for all $K \geq 0$,

$$
\begin{gathered}
f_{1}(K)=\int_{0}^{\infty} \rho(K+y) d y \leq \frac{32}{\pi K^{3}} \\
\int_{0}^{\infty} f_{1}(u-K) d u \leq K+\int_{w=0}^{\infty} \min \left(1, \frac{32}{\pi w^{3}}\right) d w=K+3 \sqrt[3]{\frac{3}{\pi}}
\end{gathered}
$$

Proof. The derivative of $f_{a, \varepsilon}$ is

$$
\begin{aligned}
\partial\left(f_{a, \varepsilon}\right)(x) & =\partial\left(1_{(-\infty, a]} * \rho_{\varepsilon}\right)(x)=\left(\partial\left(1_{(-\infty, a]}\right) * \rho_{\varepsilon}\right)(x) \\
& =\left(-\delta_{a} * \rho_{\varepsilon}\right)(x)=-\rho_{\varepsilon}(x-a),
\end{aligned}
$$

so it is indeed in $\mathscr{T}_{0}(\mathbb{R})$, and non-positive. Its Fourier transform is supported by $\left[-\frac{1}{\varepsilon}, \frac{1}{\varepsilon}\right]$, and $\left\|\partial f_{a, \varepsilon}\right\|_{\mathrm{L}^{1}}=$ $\int_{\mathbb{R}} \rho_{\varepsilon}(x) d x=1$. Then,

$$
\lim _{x \rightarrow+\infty} f_{a, \varepsilon}(x)=\lim _{x \rightarrow+\infty} f_{1}\left(\frac{x-a}{\varepsilon}\right)=\lim _{y \rightarrow+\infty} f_{1}(y),
$$

so $\lim _{x \rightarrow+\infty} f_{a, \varepsilon}(x)=0$. Since by definition $f_{1}(y)=\int_{y}^{\infty} \rho(u) d u$, the symmetry relation $f_{1}(x)=1-f_{1}(-x)$ follows from $\rho$ even; it implies the other limit statement $\lim _{x \rightarrow-\infty} f_{a, \varepsilon}(x)=1$. The inequalities in part ii) are immediate consequences of those of Lemma 17.

Let us now state a result which converts estimates on smooth test distributions into estimates of Kolmogorov distances. It already appeared in [MN15, Lemma 16], and is inspired by [Tao12, p. 87] and [Fel71, Chapter XVI, §3, Lemma 1]:

Theorem 19. Let $X$ and $Y$ be two random variables with cumulative distribution functions $F_{X}(a)=$ $\mathbb{P}[X \leq a]$ and $F_{Y}(a)=\mathbb{P}[Y \leq a]$. Assume that for some $\varepsilon>0$ and $B>0$,

$$
\sup _{a \in \mathbb{R}}\left|\mathbb{E}\left[f_{a, \varepsilon}(X)\right]-\mathbb{E}\left[f_{a, \varepsilon}(Y)\right]\right| \leq B \varepsilon
$$

We also suppose that $Y$ has a density w.r.t. Lebesgue measure that is bounded by m. Then, for every $\lambda>0$,

$$
\begin{aligned}
d_{\mathrm{Kol}}(X, Y) & =\sup _{a \in \mathbb{R}}\left|F_{X}(a)-F_{Y}(a)\right| \\
& \leq(1+\lambda)\left(B+\frac{m}{\sqrt[3]{\pi}}\left(4 \sqrt[3]{1+\frac{1}{\lambda}}+3 \sqrt[3]{3}\right)\right) \varepsilon .
\end{aligned}
$$

The choice of the parameter $\lambda$ allows one to optimize constants according to the reference law of $Y$ and to the value of $B$. A general bound is obtained by choosing $\lambda=\frac{1}{2}$; this gives after some simplifications 


$$
d_{\mathrm{Kol}}(X, Y) \leq \frac{3}{2}(B+7 m) \varepsilon
$$

which is easy to remember and manipulate.

Proof. For the convenience of the reader, we reproduce here the proof given in [MN15]. Fix a positive constant $K$, and denote $\Delta=\sup _{a \in \mathbb{R}}\left|F_{X}(a)-F_{Y}(a)\right|$ the Kolmogorov distance between $X$ and $Y$. One has

$$
\begin{aligned}
F_{X}(a)=\mathbb{E}\left[1_{X \leq a}\right] & \leq \mathbb{E}\left[f_{a+K \varepsilon, \varepsilon}(X)\right]+\mathbb{E}\left[\left(1-f_{a+K \varepsilon, \varepsilon}(X)\right) 1_{X \leq a}\right] \\
& \leq \mathbb{E}\left[f_{a+K \varepsilon, \varepsilon}(Y)\right]+\mathbb{E}\left[\left(1-f_{a+K \varepsilon, \varepsilon}(X)\right) 1_{X \leq a}\right]+B \varepsilon
\end{aligned}
$$

The second expectation writes as

$$
\begin{array}{r}
\mathbb{E}\left[\left(1-f_{a+K \varepsilon, \varepsilon}(X)\right) 1_{X \leq a}\right]=\int_{\mathbb{R}}\left(1-f_{a+K \varepsilon, \varepsilon}(x)\right) 1_{(-\infty, a]}(x) \mathbb{P}_{X}(d x) \\
=-\int_{\mathbb{R}}\left(\left(1-f_{a+K \varepsilon, \varepsilon}(x)\right) 1_{(-\infty, a]}(x)\right)^{\prime} F_{X}(x) d x=A_{1}+A_{2},
\end{array}
$$

where $A_{1}=\left(1-f_{a+K \varepsilon, \varepsilon}(a)\right) F_{X}(a), A_{2}=\int_{\mathbb{R}} f_{a+K \varepsilon, \varepsilon}^{\prime}(x) 1_{(-\infty, a]}(x) F_{X}(x) d x$. Indeed, in the space of tempered distributions, $\left(\left(1-f_{a+K \varepsilon, \varepsilon}(x)\right) 1_{(-\infty, a]}(x)\right)^{\prime}=-\left(1-f_{a+K \varepsilon, \varepsilon}(x)\right) \delta_{a}(x)-f_{a+K \varepsilon, \varepsilon}^{\prime}(x) 1_{(-\infty, a]}(x)$. We evaluate the two terms $A_{1}$ and $A_{2}$ as follows:

- Since $F_{X}(a) \leq F_{Y}(a)+\Delta$,

$$
\begin{aligned}
A_{1} & \leq\left(1-f_{a+K \varepsilon, \varepsilon}(a)\right) F_{Y}(a)+\left(1-f_{a+K \varepsilon, \varepsilon}(a)\right) \Delta \\
& \leq \int_{\mathbb{R}}\left(1-f_{a+K \varepsilon, \varepsilon}(x)\right) \delta_{a}(x) F_{Y}(x) d x+\left(1-f_{1}(-K)\right) \Delta .
\end{aligned}
$$

- For $A_{2}$, since $F_{X}(x) \geq F_{Y}(x)-\Delta$ and the derivative of $f_{a+K \varepsilon, \varepsilon}$ is negative, an upper bound is obtained as follows:

$$
\begin{aligned}
A_{2} & \leq \int_{\mathbb{R}} f_{a+K \varepsilon, \varepsilon}^{\prime}(x) 1_{(-\infty, a]}(x) F_{Y}(x) d x-\Delta \int_{\mathbb{R}} f_{a+K \varepsilon, \varepsilon}^{\prime}(x) 1_{(-\infty, a]}(x) d x \\
& =\int_{\mathbb{R}} f_{a+K \varepsilon, \varepsilon}^{\prime}(x) 1_{(-\infty, a]}(x) F_{Y}(x) d x+\left(1-f_{a+K \varepsilon, \varepsilon}(a)\right) \Delta \\
& =\int_{\mathbb{R}} f_{a+K \varepsilon, \varepsilon}^{\prime}(x) 1_{(-\infty, a]}(x) F_{Y}(x) d x+\left(1-f_{1}(-K)\right) \Delta .
\end{aligned}
$$

Therefore, by gathering the bounds on $A_{1}$ and $A_{2}$, we get

$$
\mathbb{E}\left[\left(1-f_{a+K \varepsilon, \varepsilon}(X)\right) 1_{X \leq a}\right] \leq \mathbb{E}\left[\left(1-f_{a+K \varepsilon, \varepsilon}(Y)\right) 1_{Y \leq a}\right]+2\left(1-f_{1}(-K)\right) \Delta .
$$

On the other hand, if $m$ is a bound on the density $f_{Y}$ of $Y$, then

$$
\begin{aligned}
\mathbb{E}\left[f_{a+K \varepsilon, \varepsilon}(Y) 1_{Y \geq a}\right] & =\int_{a}^{\infty} f_{a+K \varepsilon, \varepsilon}(y) f_{Y}(y) d y \\
& \leq m \int_{a}^{\infty} f_{\varepsilon}(y-a-K \varepsilon) d y=m \int_{0}^{\infty} f_{\varepsilon}(y-K \varepsilon) d y \\
& \leq m \varepsilon \int_{0}^{\infty} f_{1}(u-K) d u \leq m \varepsilon\left(K+3 \sqrt[3]{\frac{3}{\pi}}\right) ;
\end{aligned}
$$

and

$$
\mathbb{E}\left[f_{a+K \varepsilon, \varepsilon}(Y)\right] \leq \mathbb{E}\left[f_{a+K \varepsilon, \varepsilon}(Y) 1_{Y \leq a}\right]+m\left(K+3 \sqrt[3]{\frac{3}{\pi}}\right) \varepsilon
$$


Putting together Eqs. (2), (3) and (4), we get

$$
F_{X}(a) \leq F_{Y}(a)+\left(B+m\left(K+3 \sqrt[3]{\frac{3}{\pi}}\right)\right) \varepsilon+\frac{64}{\pi K^{3}} \Delta .
$$

Similarly, $F_{X}(a) \geq F_{Y}(a)-\left(B+m\left(K+3 \sqrt[3]{\frac{3}{\pi}}\right)\right) \varepsilon-\frac{64}{\pi K^{3}} \Delta$, so in the end

$$
\Delta=\sup _{a \in \mathbb{R}}\left|F_{X}(a)-F_{Y}(a)\right| \leq\left(B+m\left(K+3 \sqrt[3]{\frac{3}{\pi}}\right)\right) \varepsilon+\frac{64}{\pi K^{3}} \Delta .
$$

As this is true for every $K$, setting $K=4 \sqrt[3]{\frac{1+\lambda}{\pi \lambda}}$ with $\lambda>0$, one obtains

$$
\Delta \leq(1+\lambda)\left(B+\frac{m}{\sqrt[3]{\pi}}\left(4 \sqrt[3]{1+\frac{1}{\lambda}}+3 \sqrt[3]{3}\right)\right) \varepsilon
$$

In the next Section 2.5, we shall combine Theorem 19 and the estimates on smooth test distributions given by Proposition 16 to get a Berry-Esseen type bound on the Kolmogorov distances in the setting of mod- $\phi$ convergence.

\subsection{Bounds on the Kolmogorov distance}

We are now ready to get an estimate for the Komogorov distance under a zone of control hypothesis.

Theorem 20. Fix a reference stable distribution $\phi_{c, \alpha, \beta}$ and consider a sequence $\left(X_{n}\right)_{n \in \mathbb{N}}$ of random variables with a zone of control $\left[-K\left(t_{n}\right)^{\gamma}, K\left(t_{n}\right)^{\gamma}\right]$ of index $(v, w)$. Assume in addition that $\gamma \leq \frac{v-1}{\alpha}$. As before, we denote $Y$ a random variable with law $\phi_{c, \alpha, \beta}$, and $Y_{n}$ the renormalization of $X_{n}$ as in Proposition 3. Then, there exists a constant $C\left(\alpha, c, v, K, K_{1}\right)$ such that

$$
d_{\mathrm{Kol}}\left(Y_{n}, Y\right) \leq C\left(\alpha, c, v, K, K_{1}\right) \frac{1}{\left(t_{n}\right)^{1 / \alpha+\gamma}} .
$$

The constant $C\left(\alpha, c, v, K, K_{1}\right)$ is explicitly given by

$$
\min _{\lambda>0}\left(\frac{1+\lambda}{\alpha \pi c}\left(\frac{2^{\frac{v}{\alpha}} \Gamma\left(\frac{v}{\alpha}\right) K_{1}}{c^{v-1}}+\frac{\Gamma\left(\frac{1}{\alpha}\right)}{\sqrt[3]{\pi} K}\left(4 \sqrt[3]{1+\frac{1}{\lambda}}+3 \sqrt[3]{3}\right)\right)\right) .
$$

Note that the additional hypothesis $\gamma \leq \frac{v-1}{\alpha}$ can always be ensured by decreasing $\gamma$ (but this makes the resulting bound weaker).

Proof. We apply Proposition 16 with the smooth test distributions $f_{n}=f_{a, \varepsilon_{n}}$, with $\varepsilon_{n}:=\frac{1}{K\left(t_{n}\right)^{1 / \alpha+\gamma}}$; we know that $\left\|\partial f_{n}\right\|_{\mathrm{L}^{1}}=1$ and that $\widehat{f_{n}}$ is supported by the zone $\left[-K\left(t_{n}\right)^{1 / \alpha+\gamma}, K\left(t_{n}\right)^{1 / \alpha+\gamma}\right]$, so that

$$
\left|\mathbb{E}\left[f_{a, \varepsilon_{n}}\left(Y_{n}\right)\right]-\mathbb{E}\left[f_{a, \varepsilon_{n}}(Y)\right]\right| \leq C_{2}(c, \alpha, v) \frac{K_{1}}{\left(t_{n}\right)^{v / \alpha}} \leq \frac{2^{\frac{v}{\alpha}} \Gamma\left(\frac{v}{\alpha}\right) K_{1} K}{\alpha \pi c^{v}} \varepsilon_{n} .
$$

This allows to apply Theorem 19 with a constant

$$
B=\frac{2^{\frac{v}{\alpha}} \Gamma\left(\frac{v}{\alpha}\right) K_{1} K}{\alpha \pi c^{v}},
$$

and with $\varepsilon=\varepsilon_{n}=\frac{1}{K\left(t_{n}\right)^{1 / \alpha+\gamma}}$. Indeed, note that the density of the law of $Y$ is bounded by 


$$
m=\frac{1}{2 \pi}\left\|\mathrm{e}^{\eta(\mathrm{i} \xi)}\right\|_{\mathrm{L}^{1}}=\frac{1}{\alpha \pi c} \Gamma\left(\frac{1}{\alpha}\right) .
$$

Remark 21. Suppose $\alpha=2, c=\frac{1}{\sqrt{2}}$ (mod-Gaussian convergence), and $v=w=3$. The maximal value allowed for the exponent $\gamma$ in the size of the zone of control is then $\gamma=1$, and later we shall encounter many examples of this situation. Then, we obtain

$$
d_{\mathrm{Kol}}\left(Y_{n}, Y\right) \leq \frac{1+\lambda}{\sqrt{2 \pi}}\left(2^{\frac{3}{2}} K_{1}+\frac{1}{\sqrt[3]{\pi} K}\left(4 \sqrt[3]{1+\frac{1}{\lambda}}+3 \sqrt[3]{3}\right)\right) \frac{1}{\left(t_{n}\right)^{3 / 2}} .
$$

In Section 4, we shall give conditions on cumulants of random variables that lead to mod-Gaussian convergence with a zone of control of size $O\left(t_{n}\right)$ and with index $(3,3)$, so that (5) holds. We shall then choose $K, K_{1}$ and $\lambda$ to make the constant in the right-hand side as small as possible.

Remark 22. In the general case, taking $\lambda=\frac{1}{2}$ in Theorem 20 leads to the inequality

$$
d_{\mathrm{Kol}}\left(Y_{n}, Y\right) \leq C_{3}\left(\alpha, c, v, K_{1}, K\right) \frac{1}{\left(t_{n}\right)^{1 / \alpha+\gamma}},
$$

where $C_{3}\left(\alpha, c, v, K_{1}, K\right)=\frac{3}{2 \pi \alpha c}\left(\frac{2^{\frac{v}{\alpha}} \Gamma\left(\frac{v}{\alpha}\right) K_{1}}{c^{v-1}}+\frac{7 \Gamma\left(\frac{1}{\alpha}\right)}{K}\right)$.

\section{Examples with an explicit Fourier transform}

\subsection{Sums of independent random variables}

As a direct application of Theorem 20, one recovers the classical Berry-Esseen estimates. Let $\left(A_{n}\right)_{n \in \mathbb{N}}$ be a sequence of centered i.i.d. random variables with a third moment. We denote $\mathbb{E}\left[\left(A_{i}\right)^{2}\right]=\sigma^{2}$ and $\mathbb{E}\left[\left|A_{i}\right|^{3}\right]=\rho$. Set $S_{n}=\sum_{i=1}^{n} A_{i}, X_{n}=S_{n} /\left(\sigma n^{1 / 3}\right)$,

$$
t_{n}=n^{1 / 3} \quad ; \quad K=\frac{\sigma^{3}}{\rho} \quad ; \quad v=w=3 \quad ; \quad \gamma=1 .
$$

Notice that $K \leq 1$ as a classical application of Hölder inequality. On the zone $\xi \in\left[-K n^{1 / 3}, K n^{1 / 3}\right]$, we have:

$$
\begin{aligned}
& \left|\theta_{n}(\xi)-1\right|=\mid\left(\mathbb{E}\left[\mathrm{e}^{\left.\mathrm{i} \xi \frac{A_{1}}{\sigma n^{1 / 3}}\right]} \mathrm{e}^{\frac{\xi^{2}}{2 n^{2 / 3}}}\right)^{n}-1 \mid\right. \\
& \quad \leq n \mid \mathbb{E}\left[\mathrm{e}^{\left.\mathrm{i} \xi \frac{A_{1}}{\sigma n^{1 / 3}}\right]} \mathrm{e}^{\frac{\xi^{2}}{2 n^{2 / 3}}}-1 \mid\left(\max \left(\mid \mathbb{E}\left[\mathrm{e}^{\left.\mathrm{i} \xi \frac{A_{1}}{\sigma n^{1 / 3}}\right]} \mathrm{e}^{\frac{\xi^{2}}{2 n^{2 / 3}}} \mid, 1\right)\right)^{n-1} .\right.\right.
\end{aligned}
$$

For any $t,\left|\mathrm{e}^{\mathrm{i} t}-1-\mathrm{i} t+\frac{t^{2}}{2}\right| \leq \frac{\left|t^{3}\right|}{6}$, so

$$
\begin{aligned}
& \left|\mathbb{E}\left[\mathrm{e}^{\left.\mathrm{i} \xi \frac{A_{1}}{\sigma n^{1 / 3}}\right]}\right] \mathrm{e}^{\frac{\xi^{2}}{2 n^{2 / 3}}}-1\right| \\
& \leq\left|\mathbb{E}\left[\mathrm{e}^{\mathrm{i} \xi \frac{A_{1}}{\sigma n^{1 / 3}}}\right]-1+\frac{\xi^{2}}{2 n^{2 / 3}}\right| \mathrm{e}^{\frac{\xi^{2}}{2 n^{2 / 3}}}+\left|\mathrm{e}^{-\frac{\xi^{2}}{2 n^{2 / 3}}}-1+\frac{\xi^{2}}{2 n^{2 / 3}}\right| \mathrm{e}^{\frac{\xi^{2}}{2 n^{2 / 3}}} \\
& \leq\left(\frac{|\xi|^{3}}{6 K n}+\frac{\xi^{4}}{8 n^{4 / 3}}\right) \mathrm{e}^{\frac{\xi^{2}}{2 n^{2 / 3}}} \leq \frac{7 \mathrm{e}^{1 / 2}}{24} \frac{|\xi|^{3}}{K n} .
\end{aligned}
$$

For the same reasons, 


$$
\begin{aligned}
\mid \mathbb{E}\left[\mathrm{e}^{\left.\mathrm{i} \xi \frac{A_{1}}{\sigma n^{1 / 3}}\right]} \mathrm{e}^{\frac{\xi^{2}}{2 n^{2 / 3}} \mid}\right. & \leq \frac{|\xi|^{3}}{6 K n} \mathrm{e}^{\frac{\xi^{2}}{2 n^{2 / 3}}}+\left(1-\frac{\xi^{2}}{2 n^{2 / 3}}\right) \mathrm{e}^{\frac{\xi^{2}}{2 n^{2 / 3}}} \\
& \leq \frac{|\xi|^{3}}{6 K n} \mathrm{e}^{1 / 2}+1 \leq \mathrm{e}^{\frac{\mathrm{e}^{1 / 2}}{6} \frac{|\xi|^{3}}{K n}}
\end{aligned}
$$

We conclude that

$$
\left|\theta_{n}(\xi)-1\right| \leq \frac{7 \mathrm{e}^{1 / 2}}{24} \frac{|\xi|^{3}}{K} \mathrm{e}^{\frac{\mathrm{e}^{1 / 2}}{6} \frac{|\xi|^{3}}{K}}
$$

on the zone of control $\left[-K n^{1 / 3}, K n^{1 / 3}\right]$. If we want Condition (Z2) to be satisfied, we need to change $K$ and set

$$
K=\frac{3}{2 \mathrm{e}^{1 / 2}} \frac{\sigma^{3}}{\rho},
$$

which is a little bit smaller than before. We then have a zone of control with constants $K_{1}=\frac{7 \mathrm{e}^{1 / 2} \rho}{24 \sigma^{3}}$ and $K_{2}=\frac{\mathrm{e}^{1 / 2} \rho}{6 \sigma^{3}}$, and the inequality $K \leq\left(\frac{c^{\alpha}}{2 K_{2}}\right)^{\frac{1}{w-\alpha}}$ is an equality. By Theorem 20,

$$
d_{\mathrm{Kol}}\left(Y_{n}, \mathcal{N}_{\mathbb{R}}\right) \leq \frac{1+\lambda}{\sqrt{2 \pi}}\left(\frac{7}{24} 2^{3 / 2} \mathrm{e}^{1 / 2}+\frac{2 \mathrm{e}^{1 / 2}}{3 \sqrt[3]{\pi}}\left(4 \sqrt[3]{1+\frac{1}{\lambda}}+3 \sqrt[3]{3}\right)\right) \frac{\rho}{\sigma^{3} \sqrt{n}}
$$

with $Y_{n}=\frac{1}{\sigma \sqrt{n}} \sum_{i=1}^{n} A_{i}$. Taking $\lambda=0.183$, we obtain a bound with a constant $C \leq 4.815$, so we recover

$$
d_{\mathrm{Kol}}\left(Y_{n}, \mathcal{N}_{\mathbb{R}}(0,1)\right) \leq 4.815 \frac{\rho}{\sigma^{3} \sqrt{n}}
$$

which is almost as good as the statement in the introduction, where a constant $C=3$ was given (the best constant known today is, as far as we know, $C=0.4748$, see [KS10]). Of course, the advantage of our method is its large range of applications, as we shall see in the next sections.

Our notion of zone of control allows one to deal with sums of random variables that are independent but not identically distributed. As an example, consider for $r<1$ a random series

$$
Z_{r}=\sum_{k=1}^{\infty} \mathcal{B}\left(r^{2 k}\right)
$$

with Bernoulli variables of parameters $r^{2 k}$ that are independent. The random variable $Z_{r}$ has the same law as the number of zeroes with module smaller than $r$ of a random analytic series $S(z)=\sum_{n=0}^{\infty} a_{n} z^{n}$, where the $a_{n}$ 's are independent standard complex Gaussian variables (see [FMN16, Section 7.1]). If $h=\frac{4 \pi r^{2}}{1-r^{2}}$ is the hyperbolic area of the disc of radius $r$ and center 0 , then we showed in loc. cit. that as $h$ goes to infinity and $r$ goes to 1 , denoting $Z_{r}=Z^{h}$, the sequence

$$
X_{h}=\frac{1}{h^{1 / 3}}\left(Z^{h}-\frac{h}{4 \pi}\right)
$$

is mod-Gaussian convergent with parameters $t_{h}=\frac{h^{1 / 3}}{8 \pi}$ and limit $\theta(\xi)=\exp \left(\frac{(\mathrm{i} \xi)^{3}}{144 \pi}\right)$. Let us compute a zone of control for this mod-Gaussian convergence. We change a bit the parameters of the mod-Gaussian convergence and take

$$
\tilde{t}_{h}=\operatorname{Var}\left(X_{h}\right)=\frac{1}{h^{2 / 3}} \sum_{k=1}^{\infty} r^{2 k}\left(1-r^{2 k}\right)=\frac{h^{1 / 3}(h+4 \pi)}{4 \pi(2 h+4 \pi)} .
$$

The precise reason for this small modification will be given in Remark 26. Then, 


$$
\theta_{h}(\xi)=\mathbb{E}\left[\mathrm{e}^{\mathrm{i} \xi X_{h}}\right] \mathrm{e}^{\frac{\tilde{t}_{h} \xi^{2}}{2}}=\prod_{k=1}^{\infty}\left(1+r^{2 k}\left(\mathrm{e}^{\frac{\mathrm{i} \xi}{h^{1 / 3}}}-1\right)\right) \mathrm{e}^{-\frac{r^{2 k_{\mathrm{i}} \xi}}{h^{1 / 3}}+\frac{r^{2 k}\left(1-r^{2 k}\right) \xi^{2}}{2 h^{2 / 3}}}
$$

Denote $\theta_{h, k}(\xi)$ the terms of the product on the right-hand side. For $|\xi| \leq \frac{h^{1 / 3}}{4}$, we are going to compute bounds on $\left|\theta_{h, k}(\xi)\right|$ and $\left|\theta_{h, k}(\xi)-1\right|$. The holomorphic function

$$
f_{\alpha}(z)=\log \left(1+\alpha\left(\mathrm{e}^{z}-1\right)\right)-\alpha z-\frac{\alpha(1-\alpha) z^{2}}{2}
$$

has its two first derivatives at 0 that vanish, and its third complex derivative is

$$
f_{\alpha}^{\prime \prime \prime}(z)=\alpha(1-\alpha) \mathrm{e}^{z} \frac{\left(1-\alpha\left(1+\mathrm{e}^{z}\right)\right)}{\left(1+\alpha\left(\mathrm{e}^{z}-1\right)\right)^{3}} .
$$

If $|\xi| \leq \frac{h^{1 / 3}}{4}$, then $\left|\mathrm{e}^{\frac{\mathrm{i} \xi}{h^{1 / 3}}}\right| \leq \mathrm{e}^{1 / 4}$ and $\left|\mathrm{e}^{\frac{\mathrm{i} \xi}{h^{1 / 3}}}-1\right| \leq \frac{1}{4} \mathrm{e}^{1 / 4} \leq \frac{1}{2}$, so

$$
\begin{aligned}
\left|\log \theta_{h, k}(\xi)\right| & \leq \frac{|\xi|^{3}}{6 h} r^{2 k}\left(1-r^{2 k}\right) \mathrm{e}^{1 / 4} \frac{1+\frac{r^{2 k}}{2}}{\left(1-\frac{1}{4} \mathrm{e}^{1 / 4} r^{2 k}\right)^{3}} \\
& \leq \frac{|\xi|^{3}}{4 h} \frac{\mathrm{e}^{1 / 4} r^{2 k}}{\left(1-\frac{1}{4} \mathrm{e}^{1 / 4}\right)^{2}} \leq \frac{|\xi|^{3} r^{2 k}}{h} .
\end{aligned}
$$

Therefore, $\left|\theta_{h, k}(\xi)\right| \leq \exp \left(\frac{\left|\xi^{3}\right| r^{2 k}}{h}\right)$ and $\left|\theta_{h, k}(\xi)-1\right| \leq \frac{\left|\xi^{3}\right| r^{2 k}}{h} \exp \left(\frac{\left|\xi^{3}\right| r^{2 k}}{h}\right)$. We then obtain on the zone $|\xi| \leq \frac{h^{1 / 3}}{4}$

$$
\left|\theta_{h}(\xi)-1\right| \leq \sum_{k=1}^{\infty}\left|\theta_{h, k}(\xi)-1\right| \prod_{j \neq k}\left|\theta_{h, j}(\xi)\right| \leq S \exp S
$$

with $S=\sum_{k=1}^{\infty} \frac{\left|\xi^{3}\right| r^{2 k}}{h}=\frac{\left|\xi^{3}\right|}{4 \pi}$. The inequalities of Condition (Z2) forces us to look at a slightly smaller zone $\xi \in\left[-\pi \widetilde{t}_{h}, \pi \widetilde{t}_{h}\right]$; then, this zone of control has index $(3,3)$ and constants $K_{1}=K_{2}=\frac{1}{4 \pi}$. We can then apply Theorem 20, and we obtain for $h$ large enough

$$
d_{\text {Kol }}\left(\frac{Z^{h}-\frac{h}{4 \pi}}{\sqrt{\operatorname{Var}\left(Z^{h}\right)}}, \mathcal{N}_{\mathbb{R}}(0,1)\right) \leq \frac{C}{\sqrt{h}}
$$

with a constant $C \leq 166$.

\subsection{Winding number of a planar Brownian motion}

In this section, we consider a standard planar Brownian motion $\left(Z_{t}\right)_{t \in \mathbb{R}_{+}}$starting from $z=1$. It is well known that, a.s., $Z_{t}$ never touches the origin. One can thus write $Z_{t}=R_{t} \mathrm{e}^{\mathrm{i} \varphi_{t}}$, for continuous functions $t \mapsto R_{t}$ and $t \mapsto \varphi_{t}$, where $\varphi_{0}=0$, see Figure 5 .

The Fourier transform of the winding number $\varphi_{t}$ was computed by Spitzer in [Spi58]:

$$
\mathbb{E}\left[\mathrm{e}^{\mathrm{i} \xi \varphi_{t}}\right]=\sqrt{\frac{\pi}{8 t}} \mathrm{e}^{-\frac{1}{4 t}}\left(I_{\frac{|\xi|-1}{2}}\left(\frac{1}{4 t}\right)+I_{\frac{|\xi|+1}{2}}\left(\frac{1}{4 t}\right)\right)
$$

where $I_{\nu}(z)=\sum_{k=0}^{\infty} \frac{1}{k ! \Gamma(\nu+k+1)}\left(\frac{z}{2}\right)^{\nu+2 k}$ is the modified Bessel function of the first kind. As a consequence, and as was noticed in [DKN15, §3.2], $\left(\varphi_{t}\right)_{t \in \mathbb{R}_{+}}$converges mod-Cauchy with parameters $\frac{\log 8 t}{2}$ and limiting function $\theta(\xi)=\sqrt{\pi} \Gamma\left(\frac{|\xi|+1}{2}\right)^{-1}$. Indeed, 


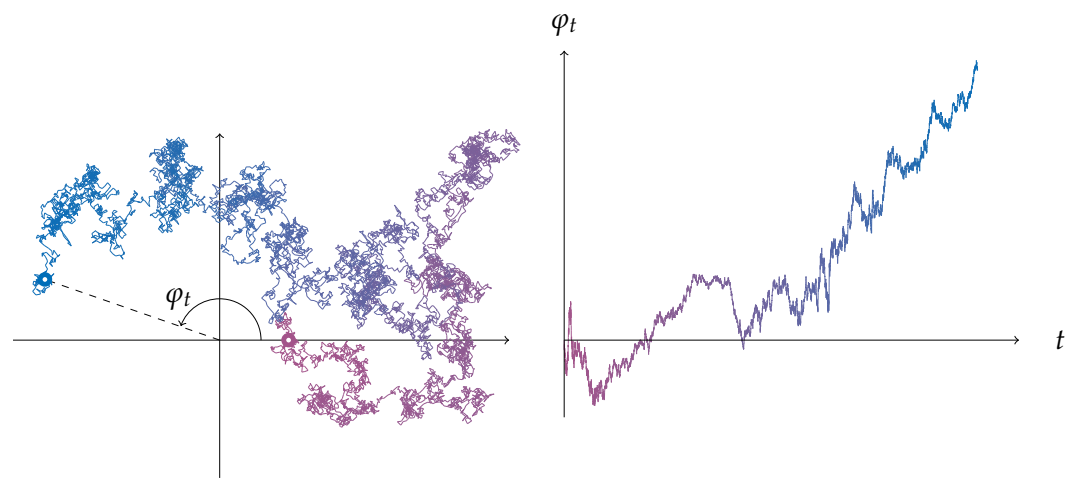

Fig. 5 Planar Brownian motion and its winding number; we will see that the latter is asymptotically mod-Cauchy.

$$
\begin{aligned}
& \mathbb{E}\left[\mathrm{e}^{\mathrm{i} \xi \varphi_{t}}\right] \exp \left(|\xi| \frac{\log 8 t}{2}\right) \\
& =\sqrt{\pi} \mathrm{e}^{-\frac{1}{4 t}}\left(\sum_{k=0}^{\infty} \frac{1}{k !}\left(\frac{1}{8 t}\right)^{2 k}\left(\frac{1}{\Gamma\left(k+\frac{|\xi|+1}{2}\right)}+\frac{1}{8 t \Gamma\left(k+\frac{|\xi|+3}{2}\right)}\right)\right),
\end{aligned}
$$

and the limit of the power series as $t$ goes to infinity is its constant term $\frac{1}{\Gamma\left(\frac{|\xi|+1}{2}\right)}$.

Here, $|\theta(\xi)-1|$ is of order $O(|\xi|)$ around 0 , since the first derivative of $\Gamma$ is not zero at $\frac{1}{2}$. Therefore, if the mod-convergence can be given a zone of control, then the index of this control will be $v=1$, which forces for Berry-Esseen estimates $\gamma \leq 0$ since $\gamma \leq \min \left(\frac{v-1}{\alpha}, \frac{1}{w-\alpha}\right)$. Conversely, for any $\xi$, notice that the function $x \mapsto \frac{1}{\Gamma\left(x+\frac{1}{2}\right)}$ has derivative bounded on $\mathbb{R}_{+}$by

$$
-\frac{\Gamma^{\prime}\left(\frac{1}{2}\right)}{\left(\Gamma\left(\frac{1}{2}\right)\right)^{2}}=1.11_{-}<\frac{2}{\sqrt{\pi}}
$$

and therefore that

$$
\begin{aligned}
\left|\theta_{t}(\xi)-1\right| & \leq 2 \mathrm{e}^{-\frac{1}{4 t}} \sum_{k=0}^{\infty} \frac{1}{k !}\left(\frac{1}{8 t}\right)^{2 k}\left(1+\frac{1}{8 t}\right) \frac{|\xi|}{2} \\
& \leq \mathrm{e}^{\left(\frac{1}{8 t}\right)^{2}-\frac{1}{4 t}}\left(1+\frac{1}{8 t}\right)|\xi| \leq|\xi|
\end{aligned}
$$

for $t$ large enough. So, in particular, one has mod-Cauchy convergence with index of control $(1,1)$, zone of control $[-K, K]$ with $K$ as large as wanted, and constants $K_{1}=1$ and $K_{2}=0$. It follows then from Theorem 20 that if $\mathcal{C}$ follows a standard Cauchy law, then

$$
d_{\text {Kol }}\left(\frac{2 \varphi_{t}}{\log 8 t}, \mathcal{C}\right) \leq \frac{4}{\log 8 t}
$$

for $t$ large enough. As far as we know, this estimate is new.

\subsection{Approximation of a stable law by compound Poisson laws}

Let $\phi_{c, \alpha, \beta}$ be a stable law; the Lévy-Khintchine formula for its exponent allows one to write 


$$
\mathrm{e}^{\eta_{c, \alpha, \beta}(\mathrm{i} \xi)}= \begin{cases}\mathrm{e}^{-|c \xi|^{2}} & \text { if } \alpha=2, \\ \exp \left(\mathrm{i} m \xi+\int_{\mathbb{R}}\left(\mathrm{e}^{\mathrm{i} \xi x}-1-1_{|x|<1} \mathrm{i} \xi x\right) \pi_{c, \alpha, \beta}(d x)\right) & \text { if } \alpha \in(0,2),\end{cases}
$$

where $\pi_{c, \alpha, \beta}(d x)$ is the Lévy measure defined for $\alpha \in(0,2)$ by

$$
\pi_{c, \alpha, \beta}(d x)=\frac{c_{+} 1_{x>0}}{x^{1+\alpha}}+\frac{c_{-} 1_{x<0}}{|x|^{1+\alpha}},
$$

with $m \in \mathbb{R}$ and $c_{+}, c_{-} \in \mathbb{R}_{+}$related to $(c, \alpha, \beta)$ by $\beta=\frac{c_{+}-c_{-}}{c_{+}+c_{-}}$and

$$
\begin{aligned}
m & = \begin{cases}\frac{c_{+}-c_{-}}{1-\alpha} & \text { if } \alpha \neq 1, \\
\left(\int_{0}^{1} \frac{\sin t-t}{t^{2}} d t+\int_{1}^{\infty} \frac{\sin t}{t^{2}} d t\right)\left(c_{+}-c_{-}\right) & \text {if } \alpha=1 ;\end{cases} \\
c_{+}+c_{-} & = \begin{cases}\frac{\alpha c^{\alpha}}{\Gamma(1-\alpha) \sin \left(\frac{\pi(1-\alpha)}{2}\right)} & \text { if } \alpha \neq 1, \\
\frac{2 c}{\pi} & \text { if } \alpha=1 .\end{cases}
\end{aligned}
$$

The proof of the Lévy-Khintchine formula in the general case of an infinitely divisible law involves the following elementary fact ( $c f$. [Sat99, Chapter 2]): if $\mu$ is infinitely divisible and $\mu=\left(\rho_{n}\right)^{* n}$ for $n \geq 1$, then the compound Poisson law $\mu_{n}$ of intensity $n \rho_{n}$, which has Fourier transform

$$
\widehat{\mu_{n}}(\xi)=\exp \left(\int_{\mathbb{R}}\left(\mathrm{e}^{\mathrm{i} x \xi}-1\right) n \rho_{n}(d x)\right),
$$

converges in law towards $\mu$; thus, any infinitely divisible law is a limit of compound Poisson laws. In the case of stable laws, this approximation result can be precised in terms of Kolmogorov distances:

Proposition 23. Let $Y$ be a random variable with stable law $\phi_{c, \alpha, \beta}$, and $Y_{n}$ be a random variable with the following compound Poisson distribution: if $\mu_{n}$ is the law of $Y_{n}$, then its Fourier transform is

$$
\widehat{\mu_{n}}(\xi)=\exp \left(\int_{\mathbb{R}}\left(\mathrm{e}^{\mathrm{i} x \xi}-1\right) n \phi_{\frac{c}{n^{1 / \alpha}}, \alpha, \beta}(d x)\right) .
$$

The Kolmogorov distance between $Y_{n}$ and $Y$ is

$$
d_{\mathrm{Kol}}\left(Y_{n}, Y\right) \leq \begin{cases}C(\alpha) n^{-1 / \alpha} & \text { if } \alpha \in(1,2], \\ C(\alpha) n^{-1} & \text { if } \alpha \in(0,1) \text { or } \alpha=1, \beta=0, \\ C^{\prime}(\log n)^{2} n^{-1} & \text { if } \alpha=1, \beta \neq 0,\end{cases}
$$

with constants $C(\alpha)$ or $C^{\prime}$ that depend only on the exponent $\alpha$.

We thus get a phase transition between the cases $\alpha>1$ and $\alpha<1$, with the case $\alpha=1$ that exhibit distinct transition behaviors according to the value of $\beta$.

Proof. Let us distinguish the following cases:

- Suppose first $\alpha \notin\{1,2\}$. The definition of $Y_{n}$ implies that

$$
\mathbb{E}\left[\mathrm{e}^{\mathrm{i} \xi Y_{n}}\right]=\widehat{\mu_{n}}(\xi)=\exp \left(n\left(\mathrm{e}^{\frac{\eta_{c, \alpha, \beta}(\mathrm{i} \xi)}{n}}-1\right)\right) .
$$

Set $X_{n}=n^{1 /(2 \alpha)} Y_{n}, t_{n}=\sqrt{n}$ and $\theta_{n}(\xi)=\mathbb{E}\left[\mathrm{e}^{\mathrm{i} \xi X_{n}}\right] \mathrm{e}^{-t_{n} \eta_{c, \alpha, \xi}(\mathrm{i} \xi)}$. We have

$$
\theta_{n}(\xi)=\exp \left(n\left(\mathrm{e}^{\frac{\eta_{c, \alpha, \beta}(\mathrm{i} \xi)}{n^{1 / 2}}}-1-\frac{\eta_{c, \alpha, \beta}(\mathrm{i} \xi)}{n^{1 / 2}}\right)\right) \text {. }
$$

On the zone $\left[-K\left(t_{n}\right)^{1 / \alpha}, K\left(t_{n}\right)^{1 / \alpha}\right]$ with $K=\frac{\left|\cos \left(\frac{\pi \alpha}{2}\right)\right|^{\frac{2}{\alpha}}}{c}$, we can use a Taylor formula with an integral form remainder: 


$$
\begin{gathered}
n\left(\mathrm{e}^{\frac{\eta_{c, \alpha, \beta}^{(\mathrm{i} \xi)}}{n^{1 / 2}}}-1-\frac{\eta_{c, \alpha, \beta}(\mathrm{i} \xi)}{n^{1 / 2}}\right)=\left(\eta_{c, \alpha, \beta}(\mathrm{i} \xi)\right)^{2}\left(\int_{0}^{1}(1-u) \mathrm{e}^{\frac{u \eta_{c, \alpha, \beta}^{(\mathrm{i} \xi)}}{n^{1 / 2}}} d u\right) \\
\left|n\left(\mathrm{e}^{\frac{\eta_{c, \alpha, \beta}(\mathrm{i} \xi)}{n^{1 / 2}}}-1-\frac{\eta_{c, \alpha, \beta}(\mathrm{i} \xi)}{n^{1 / 2}}\right)\right| \leq \frac{1}{2}\left|\eta_{c, \alpha, \beta}(\mathrm{i} \xi)\right|^{2} \leq \frac{1}{2}\left(\frac{c^{\alpha}}{\cos \left(\frac{\pi \alpha}{2}\right)}\right)^{2}|\xi|^{2 \alpha} .
\end{gathered}
$$

We thus obtain a zone of control for $\left(X_{n}\right)_{n \in \mathbb{N}}$ with $v=w=2 \alpha, \gamma=\frac{1}{\alpha}$,

$$
K_{1}=K_{2}=\frac{1}{2}\left(\frac{c^{\alpha}}{\cos \left(\frac{\pi \alpha}{2}\right)}\right)^{2}
$$

and one checks that

$$
\left(\frac{c^{\alpha}}{2 K_{2}}\right)^{\frac{1}{w-\alpha}}=\frac{\left|\cos \left(\frac{\pi \alpha}{2}\right)\right|^{\frac{2}{\alpha}}}{c}=K
$$

Since we need $\gamma \leq \min \left(\frac{1}{w-\alpha}, \frac{v-1}{\alpha}\right)$ to obtain a bound on the Kolmogorov distance (see the hypotheses of Theorem 20), this leads to a reduction of $\gamma$ when $\alpha<1$ :

$$
\gamma+\frac{1}{\alpha}= \begin{cases}\frac{2}{\alpha} & \text { if } \alpha>1 \\ 2 & \text { if } \alpha<1\end{cases}
$$

With Theorem 20, we obtain the following upper bound for $d_{\mathrm{Kol}}\left(Y_{n}, Y\right)$ :

$$
\frac{1+\lambda}{\alpha \pi}\left(\frac{2}{\left(\cos \left(\frac{\pi \alpha}{2}\right)\right)^{2}}+\frac{\Gamma\left(\frac{1}{\alpha}\right)}{\sqrt[3]{\pi}\left|\cos \left(\frac{\pi \alpha}{2}\right)\right|^{2 / \alpha}}\left(4 \sqrt[3]{1+\frac{1}{\lambda}}+3 \sqrt[3]{3}\right)\right) \frac{1}{n^{\frac{\gamma}{2}+\frac{1}{2 \alpha}}},
$$

Then, any choice of $\lambda>0$ gives a constant $C(\alpha)$ that depends only on $\alpha$.

- When $\alpha=2$, the result follows from the usual Berry-Esseen estimates, since $\sqrt{n} Y_{n}$ has the law of a sum of $n$ independent random variables with same law and finite variance and third moment.

- If $\alpha=1$ and $\beta=0$, then the same computations as above can be performed with a constant $K=\frac{1}{c}$, $v=w=2, \gamma=1$,

$$
K_{1}=K_{2}=\frac{c^{2}}{2}
$$

and this leads to

$$
d_{\mathrm{Kol}}\left(Y_{n}, Y\right) \leq \frac{1+\lambda}{\pi}\left(2+\frac{1}{\sqrt[3]{\pi}}\left(4 \sqrt[3]{1+\frac{1}{\lambda}}+3 \sqrt[3]{3}\right)\right) \frac{1}{n}
$$

and a constant $C=3.04$ when $\lambda=0.2$.

- Let us finally treat the case $\alpha=1, \beta \neq 0$. Recall that we then have $\eta_{c, \alpha, \beta}(\mathrm{i} \xi)=-|c \xi|(1+$ $\left.\frac{2 \mathrm{i} \beta}{\pi} \operatorname{sgn}(\xi) \log |\xi|\right)$. We choose $t_{n}$ such that $t_{n} \log t_{n}=\sqrt{n}$, and set

$$
X_{n}=t_{n} Y_{n}+\frac{2 c \beta}{\pi} \sqrt{n}
$$

We then have

$$
\begin{aligned}
\theta_{n}(\xi) & =\mathbb{E}\left[\mathrm{e}^{\mathrm{i} \xi X_{n}}\right] \mathrm{e}^{-t_{n} \eta_{c, \alpha, \beta}(\mathrm{i} \xi)} \\
& =\exp \left(\frac{2 c \beta \mathrm{i} \xi}{\pi} t_{n} \log t_{n}+n\left(\mathrm{e}^{\frac{\eta_{c, \alpha, \beta}\left(\mathrm{i} t_{n} \xi\right)}{n}}-1\right)-t_{n} \eta_{c, \alpha, \beta}(\mathrm{i} \xi)\right) \\
& =\exp \left(n\left(\mathrm{e}^{\frac{\eta_{c, \alpha, \beta}\left(\mathrm{i} t_{n} \xi\right)}{n}}-1-\frac{\eta_{c, \alpha, \beta}\left(\mathrm{i} t_{n} \xi\right)}{n}\right)\right),
\end{aligned}
$$


and the Taylor formula with integral remainder yields:

$$
\begin{aligned}
\left|n\left(\mathrm{e}^{\frac{\eta_{c, \alpha, \beta}\left(\mathrm{i} t_{n} \xi\right)}{n}}-1-\frac{\eta_{c, \alpha, \beta}\left(\mathrm{i} t_{n} \xi\right)}{n}\right)\right| & \leq \frac{1}{2 n}\left|\eta_{c, \alpha, \beta}\left(\mathrm{i} t_{n} \xi\right)\right|^{2} \\
& \leq \frac{c^{2}|\xi|^{2}}{2}\left(\frac{1+\frac{4}{\pi^{2}}\left(\log \left|t_{n} \xi\right|\right)^{2}}{\left(\log t_{n}\right)^{2}}\right) .
\end{aligned}
$$

On the zone $\left[-\frac{t_{n}}{2 c}, \frac{t_{n}}{2 c}\right]$, we thus have

$$
\begin{aligned}
& \left|n\left(\mathrm{e}^{\frac{\eta_{c, \alpha, \beta}\left(\mathrm{i} t_{n} \xi\right)}{n}}-1-\frac{\eta_{c, \alpha, \beta}\left(\mathrm{i} t_{n} \xi\right)}{n}\right)\right| \\
& \leq \frac{c^{2}|\xi|^{2}}{2}\left(\frac{1+\frac{4}{\pi^{2}}\left(2 \log t_{n}-\log 2 c\right)^{2}}{\left(\log t_{n}\right)^{2}}\right) \\
& \leq c^{2}|\xi|^{2} \quad \text { for } t_{n} \text { large enough. }
\end{aligned}
$$

So, there is a zone of control with constants $K_{1}=K_{2}=c^{2}, v=w=2$ and $\gamma=1$, and $K=\frac{1}{2 c}$. We thus get as before an estimate of $d_{\mathrm{Kol}}\left(Y_{n}, Y\right)$ of order $\mathcal{O}\left(\left(t_{n}\right)^{-2}\right)$, and since $\left(t_{n} \log t_{n}\right)^{2}=n,\left(t_{n}\right)^{2}$ is asymptotically equivalent to $\frac{4 n}{\log ^{2} n}$.

\subsection{Convergence of Ornstein-Uhlenbeck processes to stable laws}

Another way to approximate a stable law $\phi_{c, \alpha, \beta}$ is by using the marginales of a random process of Ornstein-Uhlenbeck type. Consider more generally a self-decomposable law $\phi$ on $\mathbb{R}$, that is an infinitely divisible distribution such that for any $b \in(0,1)$, there exists a probability measure $p_{b}$ on $\mathbb{R}$ such that

$$
\widehat{\phi}(\xi)=\widehat{\phi}(b \xi) \widehat{p}_{b}(\xi) ;
$$

see [Sat99, Chapter 3, Definition 15.1]. In Equation (6), the laws $p_{b}$ are the marginale laws of certain Markov processes. Fix a Lévy-Khintchine triplet $\left(l \in \mathbb{R}, \nu^{2} \in \mathbb{R}_{+}, \rho\right)$ with $\rho$ probability measure on $\mathbb{R} \backslash\{0\}$ that integrates $\min \left(1,|x|^{2}\right)$, and consider the Lévy process $\left(Z_{t}\right)_{t \in \mathbb{R}_{+}}$associated to this triplet:

$$
\begin{aligned}
\mathbb{E}\left[\mathrm{e}^{\mathrm{i} \xi Z_{t}}\right] & =\exp (t \psi(\mathrm{i} \xi)) \\
& =\exp \left(t\left(\mathrm{i} l \xi-\frac{\nu^{2} \xi^{2}}{2}+\int_{\mathbb{R}}\left(\mathrm{e}^{\mathrm{i} \xi x}-1-1_{|x|<1} \mathrm{i} \xi x\right) \rho(d x)\right)\right) .
\end{aligned}
$$

The Ornstein-Uhlenbeck process with triplet $\left(l, \nu^{2}, \rho\right)$, speed $v$ and starting point $x$ is the solution $\left(U_{t}\right)_{t \geq 0}$ of the stochastic differential equation

$$
U_{t}=\mathrm{e}^{-v t} x+\int_{0}^{t} \mathrm{e}^{-v(t-s)} d Z_{s}
$$

The Ornstein-Uhlenbeck process $\left(U_{t}\right)_{t \geq 0}$ can be shown to be a Markov process whose transition kernel $\left(P_{t}(x, d y)\right)_{t \geq 0}$ satisfies:

$$
\left.\widehat{P_{t}(x, \cdot}\right)(\xi)=\int_{\mathbb{R}} \mathrm{e}^{\mathrm{i} \xi y} P_{t}(x, d y)=\exp \left(\mathrm{i} \xi \mathrm{e}^{-v t} x+\int_{0}^{t} \psi\left(\mathrm{i} \xi \mathrm{e}^{-v s}\right) d s\right)
$$

see [Sat99, Lemma 17.1]. The connection with self-decomposable laws is provided by:

Theorem 24 (Sato-Yamazato, 1983). For any self-decomposable law $\phi$ and any fixed speed $v$, there exists a unique Lévy-Khintchine triplet $\left(l, \nu^{2}, \rho\right)$ with $\int_{|x| \geq 1} \log |x| \rho(d x)<+\infty$, such that the associated Ornstein-Uhlenbeck process $\left(U_{t}\right)_{t \geq 0}$ with speed $v$ satisfies: 


$$
\forall x \in \mathbb{R}, P_{t}(x, \cdot) \rightarrow \phi .
$$

If $\psi(\mathrm{i} \xi)$ is the exponent associated to $\left(l, \nu^{2}, \rho\right)$, then

$$
\widehat{\phi}(\xi)=\exp \left(\int_{s=0}^{\infty} \psi\left(\mathrm{i} \xi \mathrm{e}^{-v s}\right) d s\right) .
$$

We refer to [SY83] and [Sat99, Theorem 17.5]. In the setting of Theorem 24, one has the relation

$$
\widehat{\phi}(\xi)=\widehat{\phi}\left(\mathrm{e}^{-v t} \xi\right)\left(\widehat{P_{t}(x, \cdot)}(\xi) \widehat{\delta_{-\mathrm{e}^{-v t} x}}(\xi)\right),
$$

so if $b \in(0,1)$, setting $b=\mathrm{e}^{-v t}$, one recovers $p_{b}$ as the law of $U_{t}-\mathrm{e}^{-v t} x$, where $\left(U_{t}\right)_{t \in \mathbb{R}_{+}}$is the OrnsteinUhlenbeck process that converges in distribution to $\phi$ and that has speed $v$ and starting point $x$.

Suppose that $\phi=\phi_{c, \alpha, \beta}$ is a stable law. Then, the previous computations can be reinterpreted in the framework of mod- $\phi$ convergence. We set

$$
\theta(\xi)=\frac{\widehat{\delta_{x}}(\xi)}{\widehat{\phi}(\xi)}=\exp (\mathrm{i} \xi x-\eta(\mathrm{i} \xi))
$$

and

$$
X_{t}= \begin{cases}\mathrm{e}^{v t} U_{t} & \text { if } \alpha \neq 1 \\ \mathrm{e}^{v t}\left(U_{t}-\frac{2 c \beta v t}{\pi}\right) & \text { if } \alpha=1\end{cases}
$$

Then,

$$
\begin{aligned}
\mathbb{E}\left[\mathrm{e}^{\mathrm{i} \xi X_{t}}\right] & = \begin{cases}\widehat{\mu}\left(\mathrm{e}^{v t} \xi\right) \theta(\xi) & \text { if } \alpha \neq 1, \\
\widehat{\mu}\left(\mathrm{e}^{v t} \xi\right) \mathrm{e}^{-\mathrm{i} \xi \mathrm{e}^{v t} \frac{2 c \beta v t}{\pi}} \theta(\xi) & \text { if } \alpha=1,\end{cases} \\
& =\mathrm{e}^{\mathrm{e}^{\alpha v t} \eta(\mathrm{i} \xi)} \theta(\xi),
\end{aligned}
$$

so $\left(X_{t}\right)_{t \geq 0}$ converges mod- $\phi$ with parameters $\mathrm{e}^{\alpha v t}$, and with limit equal to the residue $\theta(\xi)$. Note that $\theta_{t}=\theta$ for any $t \geq 0$, so we are in a special situation where the residues are constant (time-independent). Assuming that $x \neq 0$, one has for any $\xi \in \mathbb{R}$

$$
|\theta(\xi)-1| \leq \begin{cases}K_{1}|\xi| \exp \left(K_{2}|\xi|^{\alpha}\right) & \text { if } \alpha \in(1,2] \\ K_{1}|\xi|^{\alpha} \exp \left(K_{2}|\xi|^{\alpha}\right) & \text { if } \alpha \in(0,1) \text { or } \alpha=1, \beta=0 \\ K_{1}|\xi| \log |\xi| \exp \left(K_{2}|\xi|\right) & \text { if } \alpha=1, \beta \neq 0\end{cases}
$$

For the two first cases, the condition $\gamma \leq \min \left(\frac{1}{w-\alpha}, \frac{v-1}{\alpha}\right)$ in Theorem 20 imposes the following choices of $\gamma$ when computing Berry-Esseen estimates: $\gamma=\frac{\alpha-1}{\alpha}$ when $\alpha \leq 1$, and $\gamma=0$ when $\alpha \geq 1$. In these cases, one obtains:

$$
d_{\mathrm{Kol}}\left(U_{t}, \phi_{c, \alpha, \beta}\right)= \begin{cases}O\left(\mathrm{e}^{-v t}\right) & \text { if } \alpha \in(1,2], \\ O\left(\mathrm{e}^{-\alpha v t}\right) & \text { if } \alpha \in(0,1) \text { or } \alpha=1, \beta=0 .\end{cases}
$$

Because of the term $\log |\xi|$, the last case does not exactly fit the framework of zones of control, but it is easy to adapt the proofs and one gets an estimate $O\left(v t \mathrm{e}^{-v t}\right)$. On the other hand, when $x=0$, the only difference with the previous discussion is the case $\alpha \in(1,2]$, where we obtain

$$
|\theta(\xi)-1| \leq K_{1}|\xi|^{\alpha} \exp \left(K_{2}|\xi|^{\alpha}\right)
$$

and by Theorem $20, d_{\mathrm{Kol}}\left(U_{t}, \phi_{c, \alpha, \beta}\right)=O\left(\mathrm{e}^{-\alpha v t}\right)$, choosing $\gamma=\frac{\alpha-1}{\alpha}$. So, to summarise:

Proposition 25. Let $Y$ be a random variable with stable law $\phi_{c, \alpha, \beta}$, and $\left(U_{t}\right)_{t \geq 0}$ be the corresponding Ornstein-Uhlenbeck process with starting point $x$ and speed $v$. We have: 


$$
d_{\mathrm{Kol}}\left(U_{t}, Y\right)= \begin{cases}O\left(\mathrm{e}^{-v t}\right) & \text { if } \alpha \in(1,2], x \neq 0, \\ O\left(\mathrm{e}^{-\alpha v t}\right) & \text { if } \alpha \in(0,1) \text { or } \alpha=1, \beta=0 \text { or } \alpha \in(1,2], x=0, \\ O\left(v t \mathrm{e}^{-v t}\right) & \text { if } \alpha=1, \beta \neq 0,\end{cases}
$$

with constants in the $O(\cdot)$ depending only on $x$ and $\alpha$.

\subsection{Logarithms of characteristic polynomials of random matrices}

In [KN12, Sections 3 and 4] and [FMN16, Section 7.5], the mod-Gaussian convergence of the following random variables was proven:

\begin{tabular}{|c|c|c|c|}
\hline random matrix $M_{n}$ & random variable $X_{n}$ & parameters $t_{n}$ & residue $\theta(\xi)$ \\
\hline \hline Haar $(\mathrm{U}(n))$ & $\operatorname{Re}\left(\log \operatorname{det}\left(I_{n}-M_{n}\right)\right)$ & $\frac{\log n}{2}$ & $\frac{\left(G\left(1+\frac{\mathrm{i} \xi}{2}\right)\right)^{2}}{G(1+\mathrm{i} \xi)}$ \\
$\mathrm{Haar}(\mathrm{USp}(n))$ & $\log \operatorname{det}\left(I_{2 n}-M_{n}\right)-\frac{1}{2} \log \frac{\pi n}{2}$ & $\log \frac{n}{2}$ & $\frac{G\left(\frac{3}{2}\right)}{G\left(\frac{3}{2}+\mathrm{i} \xi\right)}$ \\
$\mathrm{Haar}(\mathrm{SO}(2 n))$ & $\log \operatorname{det}\left(I_{2 n}-M_{n}\right)-\frac{1}{2} \log \frac{8 \pi}{n}$ & $\log \frac{n}{2}$ & $\frac{G\left(\frac{1}{2}\right)}{G\left(\frac{1}{2}+\mathrm{i} \xi\right)}$ \\
\hline
\end{tabular}

Table 1 Mod-Gaussian convergence of the characteristic polynomials of Haar-distributed random matrices in compact Lie groups.

Here, $G$ is Barnes' function, which is the unique entire solution of the equations $G(1)=1$ and $G(z+1)=$ $G(z) \Gamma(z)$. Moreover, the mod-Gaussian convergence holds in fact on an half-plane $H=\{z \in \mathbb{C} \mid \operatorname{Re}(z)>$ $-\alpha\}$. In the sequel, we denote $X_{n}^{\mathrm{A}}, X_{n}^{\mathrm{C}}$ and $X_{n}^{\mathrm{D}}$ the mod-Gaussian convergent random variables, according to the type of the classical group (A for unitary groups, C for compact symplectic groups and D for even orthogonal groups). Before computing zones of control for these variables, let us make the following essential remark:

Remark 26. Let $\left(X_{n}\right)_{n \in \mathbb{N}}$ be a sequence of random variables that is mod-Gaussian convergent on a domain $D \subset \mathbb{C}$ which contains a neighborhood of 0 (this ensures that $\theta_{n}$ and all its derivatives converge towards those of $\theta)$. We denote $\left(t_{n}\right)_{n \in \mathbb{N}}$ the parameters of mod-Gaussian convergence of $\left(X_{n}\right)_{n \in \mathbb{N}}$. Then, without generality, one can assume $\theta_{n}^{\prime}(0)=\theta_{n}^{\prime \prime}(0)=0$ and $\theta^{\prime}(0)=\theta^{\prime \prime}(0)=0$. Indeed, set

$$
\widetilde{X}_{n}=X_{n}+\mathrm{i} \theta_{n}^{\prime}(0) \quad ; \quad \widetilde{t}_{n}=t_{n}-\theta_{n}^{\prime \prime}(0) .
$$

We then have

$$
\widetilde{\theta}_{n}(\xi):=\mathbb{E}\left[\mathrm{e}^{\mathrm{i} \xi \widetilde{X}_{n}}\right] \mathrm{e}^{\widetilde{t}_{n} \frac{\xi^{2}}{2}}=\theta_{n}(\xi) \mathrm{e}^{-\theta_{n}^{\prime}(0) \xi-\theta_{n}^{\prime \prime}(0) \frac{\xi^{2}}{2}}
$$

and this new residue satisfies $\widetilde{\theta}_{n}^{\prime}(0)=\widetilde{\theta}_{n}^{\prime \prime}(0)=0$. For the construction of zones of control, it allows us to force $v=3$, up to a translation of $X_{n}$ and of the parameter $t_{n}$.

In the following, we only treat the case of unitary groups, the two other cases being totally similar (one could also look at the imaginary part of the log-characteristic polynomial). There is an exact formula for the Fourier transform of $X_{n}^{\mathrm{A}}[\mathrm{KS} 00$, Formula (71)]:

$$
\mathbb{E}\left[\mathrm{e}^{\mathrm{i} \xi X_{n}^{\mathrm{A}}}\right]=\prod_{k=1}^{n} \frac{\Gamma(k) \Gamma(k+\mathrm{i} \xi)}{\left(\Gamma\left(k+\frac{\mathrm{i} \xi}{2}\right)\right)^{2}} .
$$

We have $\mathbb{E}\left[X_{n}^{\mathrm{A}}\right]=0$, and

$$
\widetilde{t}_{n}=\mathbb{E}\left[\left(X_{n}^{\mathrm{A}}\right)^{2}\right]=\frac{1}{2} \sum_{k=1}^{n} \frac{\Gamma^{\prime \prime}(k)}{\Gamma(k)}-\left(\frac{\Gamma^{\prime}(k)}{\Gamma(k)}\right)^{2}=\frac{1}{2} \sum_{k=1}^{n} \psi_{1}(k),
$$


where $\psi_{1}(z)$ is the trigamma function $\frac{d^{2}}{d z^{2}}(\log \Gamma(z))$, and is given on integers by the remainder of the series $\zeta(2)$ :

$$
\psi_{1}(k)=\sum_{m=k}^{\infty} \frac{1}{m^{2}}
$$

Therefore, $\widetilde{t}_{n}=\frac{1}{2} \sum_{m=1}^{\infty} \frac{\min (n, m)}{m^{2}}=\frac{1}{2}\left(\log n+\gamma+1+O\left(n^{-1}\right)\right)$. So, $\left(X_{n}^{\mathrm{A}}\right)_{n \in \mathbb{N}}$ is mod-Gaussian convergent with parameters $\left(\widetilde{t}_{n}\right)_{n \in \mathbb{N}}$ and limit

$$
\widetilde{\theta}(\xi)=\frac{\left(G\left(1+\frac{\mathrm{i} \xi}{2}\right)\right)^{2}}{G(1+\mathrm{i} \xi)} \mathrm{e}^{\frac{(\gamma+1) \xi^{2}}{4}}
$$

which satisfies $\widetilde{\theta}^{\prime}(0)=\widetilde{\theta}^{\prime \prime}(0)=0$. With these conventions, we can write the residues $\widetilde{\theta}_{n}(\xi)$ as

$$
\widetilde{\theta}_{n}(\xi)=\left(\prod_{k=1}^{n} \frac{\Gamma(k) \Gamma(k+\mathrm{i} \xi)}{\left(\Gamma\left(k+\frac{\mathrm{i} \xi}{2}\right)\right)^{2}}\right) \mathrm{e}^{\frac{\tilde{t}_{n} \xi^{2}}{2}}=\prod_{k=1}^{n}\left(\frac{\Gamma(k) \Gamma(k+\mathrm{i} \xi)}{\left(\Gamma\left(k+\frac{\mathrm{i} \xi}{2}\right)\right)^{2}} \mathrm{e}^{\frac{\psi_{1}(k) \xi^{2}}{4}}\right) .
$$

Denote $\vartheta_{k}(\xi)$ the terms of the product on the right-hand side; we use a similar strategy as in Section 3.1 for computing a zone of control. The function $\varphi_{k}(\xi)=\log \vartheta_{k}(\xi)$ vanishes at 0 , has its two first derivatives that also vanish at 0 , and therefore writes as

$$
\varphi_{k}(\xi)=\left(\int_{0}^{1} \varphi_{k}^{\prime \prime \prime}(t \xi)(1-t)^{2} d t\right) \frac{\xi^{3}}{2} .
$$

The third derivative of $\varphi_{k}(\xi)$ is given by

$$
\varphi_{k}^{\prime \prime \prime}(\xi)=-\mathrm{i} \psi_{2}(k+\mathrm{i} \xi)+\frac{\mathrm{i}}{2} \psi_{2}\left(k+\frac{\mathrm{i} \xi}{2}\right)
$$

with $\psi_{2}(z)=-2 \sum_{j=0}^{\infty} \frac{1}{(j+z)^{3}}$. As a consequence, $\varphi_{k}^{\prime \prime \prime}(\xi)$ is uniformly bounded on $\mathbb{R}$ by

$$
3 \sum_{j=0}^{\infty} \frac{1}{(j+k)^{3}} \leq \frac{3 \zeta(3)}{k^{2}}
$$

Therefore,

$$
\begin{aligned}
\left|\varphi_{k}(\xi)\right| & \leq \frac{\zeta(3)|\xi|^{3}}{2 k^{2}} \\
\left|\vartheta_{k}(\xi)\right| & \leq \mathrm{e}^{\frac{\zeta(3)|\xi|^{3}}{2 k^{2}}} ; \\
\left|\vartheta_{k}(\xi)-1\right| & \leq \frac{\zeta(3)|\xi|^{3}}{2 k^{2}} \mathrm{e}^{\frac{\zeta(3)|\xi|^{3}}{2 k^{2}}} .
\end{aligned}
$$

It follows that for any $n$ and any $\xi \in \mathbb{R},\left|\widetilde{\theta}_{n}(\xi)-1\right| \leq S \exp S$ with $S=\sum_{k=1}^{\infty} \frac{\zeta(3)|\xi|^{3}}{2 k^{2}}=\frac{3 \zeta(3)|\xi|^{3}}{\pi^{2}}$. Set $K_{1}=K_{2}=\frac{3 \zeta(3)}{\pi^{2}}$, and $K=\frac{1}{4 K_{2}}=\frac{\pi^{2}}{12 \zeta(3)}$. We have a zone of control $\left[-K t_{n}, K t_{n}\right]$ of index $(3,3)$, with constants $K_{1}$ and $K_{2}$. We conclude with Theorem 20:

Proposition 27. Let $M_{n}$ be a random unitary matrix taken according to the Haar measure. For $n$ large enough,

$$
d_{\text {Kol }}\left(\frac{\operatorname{Re}\left(\log \operatorname{det}\left(I_{n}-M_{n}\right)\right)}{\sqrt{\operatorname{Var}\left(\operatorname{Re}\left(\log \operatorname{det}\left(I_{n}-M_{n}\right)\right)\right.}}, \mathcal{N}_{\mathbb{R}}(0,1)\right) \leq \frac{C}{(\log n)^{3 / 2}}
$$

with a constant $C \leq 18$. Up to a change of the constant, the same result holds if one replaces $\operatorname{Re}\left(\log \operatorname{det}\left(I_{n}-M_{n}\right)\right)$ by $\operatorname{Im}\left(\log \operatorname{det}\left(I_{n}-M_{n}\right)\right)$, or by 


$$
\log \operatorname{det}\left(I_{2 n}-P_{n}\right)-\mathbb{E}\left[\operatorname{det}\left(I_{2 n}-P_{n}\right)\right]
$$

with $P_{n}$ Haar distributed in the unitary compact symplectic group $\operatorname{USp}(n)$ or in the even special orthogonal group $\mathrm{SO}(2 n)$.

\section{Cumulants and dependency graphs}

\subsection{Cumulants, zone of control and Kolmogorov bound}

In this section, we will see that appropriate bounds on the cumulants of a sequence of random variables $\left(S_{n}\right)_{n \in \mathbb{N}}$ imply the existence of a large zone of control for a renormalized version of $S_{n}$. In this whole section and in the next one, the reference stable law is the standard Gaussian law. We also assume that the random variables $S_{n}$ are centered.

Let us first recall the definition of cumulants. If $X$ is a real-valued random variable with exponential generating function

$$
\mathbb{E}\left[\mathrm{e}^{z X}\right]=\sum_{r=0}^{\infty} \frac{\mathbb{E}\left[X^{r}\right]}{r !} z^{r}
$$

convergent in a neighborhood of 0 , then its cumulants $\kappa^{(1)}(X), \kappa^{(2)}(X), \ldots$ are the coefficients of the series

$$
\log \mathbb{E}\left[\mathrm{e}^{z X}\right]=\sum_{r=1}^{\infty} \frac{\kappa^{(r)}(X)}{r !} z^{r}
$$

which is also well defined for $z$ in a neighborhood of 0 (see for instance [LS59]). For example, $\kappa^{(1)}(X)=$ $\mathbb{E}[X], \kappa^{(2)}(X)=\mathbb{E}\left[X^{2}\right]-\mathbb{E}[X]^{2}=\operatorname{Var}(X)$, and

$$
\kappa^{(3)}(X)=\mathbb{E}\left[X^{3}\right]-3 \mathbb{E}\left[X^{2}\right] \mathbb{E}[X]+2 \mathbb{E}[X]^{3} .
$$

We are interested in the case where cumulants can be bounded in an appropriate way.

Definition 28. Let $\left(S_{n}\right)_{n \in \mathbb{N}}$ be a sequence of (centered) real-valued random variables. We say that $\left(S_{n}\right)_{n \in \mathbb{N}}$ admits uniform bounds on cumulants with parameters $\left(D_{n}, N_{n}, A\right)$ if, for any $r \geq 2$, we have

$$
\left|\kappa^{(r)}\left(S_{n}\right)\right| \leq N_{n} r^{r-2}\left(2 D_{n}\right)^{r-1} A^{r} .
$$

In the following, it will be convenient to set $\left(\widetilde{\sigma}_{n}\right)^{2}=\frac{\operatorname{Var}\left(S_{n}\right)}{N_{n} D_{n}}$. The inequality of Definition 28 with $r=2$ gives $\left(\widetilde{\sigma}_{n}\right)^{2} \leq 2 A^{2}$.

Lemma 29. Let $\left(S_{n}\right)_{n \in \mathbb{N}}$ be a sequence with uniform bounds on cumulants with parameters $\left(D_{n}, N_{n}, A\right)$. Set

$$
X_{n}=\frac{S_{n}}{\left(N_{n}\right)^{1 / 3}\left(D_{n}\right)^{2 / 3}} \text { and } t_{n}=\left(\widetilde{\sigma}_{n}\right)^{2}\left(\frac{N_{n}}{D_{n}}\right)^{1 / 3}=\operatorname{Var}\left(X_{n}\right) .
$$

Then, we have for $\left(X_{n}\right)_{n \in \mathbb{N}}$ a zone of control $\left[-K t_{n}, K t_{n}\right]$ of index $(3,3)$, with the following constants:

$$
K=\frac{1}{(8+4 \mathrm{e}) A^{3}}, \quad K_{1}=K_{2}=(2+\mathrm{e}) A^{3} .
$$

Proof. From the definition of cumulants, since $X_{n}$ is centered and has variance $t_{n}$, we can write 


$$
\begin{aligned}
\theta_{n}(\xi) & =\mathbb{E}\left[\mathrm{e}^{\mathrm{i} \xi X_{n}}\right] \exp \left(\frac{t_{n} \xi^{2}}{2}\right) \\
& =\exp \left(\sum_{r=3}^{\infty} \frac{\kappa^{(r)}\left(S_{n}\right)}{r !} \frac{(\mathrm{i} \xi)^{r}}{\left(N_{n}\left(D_{n}\right)^{2}\right)^{r / 3}}\right)=\exp (z),
\end{aligned}
$$

with

$$
|z| \leq \frac{1}{2} \frac{N_{n}}{D_{n}} \sum_{r=3}^{\infty} \frac{r^{r-2}}{\mathrm{e}^{r} r !}\left(\left(\frac{D_{n}}{N_{n}}\right)^{1 / 3} 2 \mathrm{e} A|\xi|\right)^{r}
$$

We set $y=\left(\frac{D_{n}}{N_{n}}\right)^{1 / 3} 2 \mathrm{e} A|\xi|$ and suppose that $y \leq 1$, that is to say that $\xi \in\left[-L\left(\frac{N_{n}}{D_{n}}\right)^{1 / 3}, L\left(\frac{N_{n}}{D_{n}}\right)^{1 / 3}\right]$ with $L=\frac{1}{2 \mathrm{e} A}$.

By Stirling's bound, the series $S(y)=\sum_{r=3}^{\infty} \frac{r^{r-2}}{r ! \mathrm{e}^{r}} y^{r}$ is convergent for any $y \in[0,1]$, and we have the inequality $S(y) \leq \frac{y^{3}}{2 \mathrm{e}^{3}(1-y)}$, which implies

$$
|z| \leq 2 \frac{(A|\xi|)^{3}}{1-\left(\frac{D_{n}}{N_{n}}\right)^{1 / 3} 2 \mathrm{e} A|\xi|} .
$$

We now consider the zone of control $\left[-K t_{n}, K t_{n}\right]$ with $K=\frac{1}{(4 \mathrm{e}+8) A^{3}}$. If $\xi$ is in this zone, then we have indeed

$$
|\xi| \leq \frac{\left(\widetilde{\sigma}_{n}\right)^{2}}{4 \mathrm{e} A^{3}}\left(\frac{N_{n}}{D_{n}}\right)^{1 / 3} \leq \frac{1}{2 \mathrm{e} A}\left(\frac{N_{n}}{D_{n}}\right)^{1 / 3}=L\left(\frac{N_{n}}{D_{n}}\right)^{1 / 3}
$$

by using the remark just before the lemma. Then,

$$
|z| \leq \frac{2 A^{3}}{1-\frac{2 \mathrm{e} A\left(\widetilde{\sigma}_{n}\right)^{2}}{(4 \mathrm{e}+8) A^{3}}}|\xi|^{3} \leq \frac{2 A^{3}}{1-\frac{\mathrm{e}}{\mathrm{e}+2}}|\xi|^{3}=(2+\mathrm{e}) A^{3}|\xi|^{3} .
$$

Thus, on the zone of control, $\left|\theta_{n}(\xi)-1\right|=\left|\mathrm{e}^{z}-1\right| \leq|z| \mathrm{e}^{|z|}$, whence a control of index $(3,3)$ and with constants

$$
K_{1}=K_{2}=(2+\mathrm{e}) A^{3} .
$$

We have chosen $K$ so that $K=\frac{1}{4 K_{2}}$, hence, the inequalities of Condition (Z2) are satisfied.

Using the results of Section 2, we obtain:

Corollary 30. Let $S_{n}$ be a sequence with a uniform bounds on cumulants with parameters $\left(D_{n}, N_{n}, A\right)$ and let $Y_{n}=\frac{S_{n}}{\sqrt{\operatorname{Var}\left(S_{n}\right)}}$. Then we have

$$
d_{\mathrm{Kol}}\left(Y_{n}, \mathcal{N}_{\mathbb{R}}(0,1)\right) \leq \frac{76.36 A^{3}}{\left(\widetilde{\sigma}_{n}\right)^{3}} \sqrt{\frac{D_{n}}{N_{n}}}
$$

Proof. We can apply Theorem 20, choosing $\gamma=1$, and $\lambda=0.193$. It yields a constant smaller than 77.911. It is however possible to get the better constant given above by redoing some of the computations in this specific setting. With $\rho>4$, set $K=\frac{1}{(4 \mathrm{e}+\rho) A^{3}}$, and $\varepsilon_{n}=\frac{1}{K\left(t_{n}\right)^{3 / 2}}$. On the zone $\xi \in\left[-\frac{1}{\varepsilon_{n}}, \frac{1}{\varepsilon_{n}}\right]$, we have a bound $\left|\theta_{n}(\xi)-1\right| \leq M|\xi|^{3} \exp \left(M|\xi|^{3}\right)$, this time with $M=\left(2+\frac{8 \mathrm{e}}{\rho}\right) A^{3}$. Hence, 


$$
\begin{aligned}
\left|\mathbb{E}\left[f_{a, \varepsilon_{n}}\left(Y_{n}\right)\right]-\mathbb{E}\left[f_{a, \varepsilon_{n}}(Y)\right]\right| & \leq \frac{M}{2 \pi\left(t_{n}\right)^{3 / 2}} \int_{-\frac{1}{\varepsilon_{n}}}^{\frac{1}{\varepsilon_{n}}}|\xi|^{2} \mathrm{e}^{-\frac{\xi^{2}}{2}+M \frac{|\xi|^{3}}{\left(t_{n}\right)^{3 / 2}}} d \xi \\
& \leq \frac{M}{2 \pi\left(t_{n}\right)^{3 / 2}} \int_{\frac{1}{\varepsilon_{n}}}^{\frac{1}{\varepsilon_{n}}}|\xi|^{2} \mathrm{e}^{-\xi^{2}\left(\frac{1}{2}-\frac{2}{\rho}\right)} d \xi \\
& \leq \frac{M}{\sqrt{2 \pi}\left(t_{n}\right)^{3 / 2}\left(1-\frac{4}{\rho}\right)^{3 / 2}}=\frac{1}{\sqrt{2 \pi}} \frac{2}{\rho\left(1-\frac{4}{\rho}\right)^{3 / 2}} \varepsilon_{n} .
\end{aligned}
$$

By Theorem 19, we get for any $\lambda>0$ :

$$
\begin{aligned}
& d_{\mathrm{Kol}}\left(\frac{S_{n}}{\sqrt{\operatorname{Var}\left(S_{n}\right)}}, \mathcal{N}_{\mathbb{R}}(0,1)\right) \\
& \leq \frac{(1+\lambda)}{\sqrt{2 \pi}}\left(\frac{2}{\rho\left(1-\frac{4}{\rho}\right)^{3 / 2}}+\frac{1}{\sqrt[3]{\pi}}\left(4 \sqrt[3]{1+\frac{1}{\lambda}}+3 \sqrt[3]{3}\right)\right) \varepsilon_{n} .
\end{aligned}
$$

The best constant is then obtained with $\rho=6.79$ and $\lambda=0.185$.

Remark 31. There is a trade-off in the bound of Corollary 30 between the a priori upper bound on $\left(\widetilde{\sigma}_{n}\right)^{2}$, and the constant $C$ that one obtains such that the Kolmogorov distance is smaller than

$$
\frac{C A^{3}}{\left(\widetilde{\sigma}_{n}\right)^{3}} \sqrt{\frac{D_{n}}{N_{n}}}
$$

This bound gets worse when $\left(\widetilde{\sigma}_{n}\right)^{2}$ is small, but on the other hand, the knowledge of a better a priori upper bound (that is precisely when $\left(\widetilde{\sigma}_{n}\right)^{2}$ is small) yields a better constant $C$. So, for instance, if one knowns that $\left(\widetilde{\sigma}_{n}\right)^{2} \leq A^{2}$ (instead of $2 A^{2}$ ), then one gets a constant $C=52.52$. A general bound that one can state and that takes into account this trade-off is:

$$
d_{\mathrm{Kol}}\left(Y_{n}, \mathcal{N}_{\mathbb{R}}(0,1)\right) \leq 27.55\left(\left(\frac{A}{\widetilde{\sigma}_{n}}\right)^{3}+\frac{A}{\widetilde{\sigma}_{n}}\right) \sqrt{\frac{D_{n}}{N_{n}}} .
$$

We are indebted to Martina Dal Borgo for having pointed out this phenomenon. In the sequel, we shall freely use this small improvement of Corollary 30.

The above corollary ensures asymptotic normality with a bound on the speed of convergence when

$$
\left(\frac{1}{\left(\widetilde{\sigma}_{n}\right)^{3}} \sqrt{\frac{D_{n}}{N_{n}}} \rightarrow 0\right) \Longleftrightarrow\left(\left(\widetilde{\sigma}_{n}\right)^{2}\left(\frac{N_{n}}{D_{n}}\right)^{1 / 3} \rightarrow+\infty\right) .
$$

Using a theorem of Janson, the asymptotic normality can be obtained under a less restrictive hypothesis, but without bound on the speed of convergence. Even if the main topic of the paper is to find bounds on the speed of convergence, we will recall the result here for the sake of completeness.

Proposition 32. As above, let $S_{n}$ be a sequence with a uniform bounds on cumulants with parameters $\left(D_{n}, N_{n}, A\right)$ and assume that

$$
\lim _{n \rightarrow \infty} \frac{\operatorname{Var}\left(S_{n}\right)}{N_{n} D_{n}}\left(\frac{N_{n}}{D_{n}}\right)^{\varepsilon}=\lim _{n \rightarrow \infty}\left(\widetilde{\sigma}_{n}\right)^{2}\left(\frac{N_{n}}{D_{n}}\right)^{\varepsilon}=+\infty
$$

for some parameter $\varepsilon \in(0,1)$. Then,

$$
\frac{S_{n}}{\sqrt{\operatorname{Var}\left(S_{n}\right)}} \rightarrow \mathcal{N}_{\mathbb{R}}(0,1)
$$


Proof. Note that the bounds on cumulants can be rewritten as

$$
\left|\kappa^{(r)}\left(Y_{n}\right)\right| \leq C_{r}\left(\frac{\operatorname{Var}\left(S_{n}\right)}{N_{n} D_{n}}\left(\frac{N_{n}}{D_{n}}\right)^{1-\frac{2}{r}}\right)^{-\frac{r}{2}}
$$

for some constant $C_{r}$. Choosing $r$ large enough so that $1-\frac{2}{r} \geq \varepsilon$, we conclude that $\kappa^{(r)}\left(Y_{n}\right) \rightarrow 0$ for $r$ large enough. This is a sufficient condition for the convergence in law towards a Gaussian distribution, see [Jan88, Theorem 1] and [Gri92].

Remark 33. Up to a change of parameters, it would be equivalent to consider bounds of the kind

$$
\left|\kappa^{(r)}\left(S_{n}\right)\right| \leq(C r)^{r} \alpha_{n}\left(\beta_{n}\right)^{r}, \quad \text { or }\left|\kappa^{(r)}\left(\frac{S_{n}}{\sqrt{\operatorname{Var}\left(S_{n}\right)}}\right)\right| \leq \frac{r !}{\Delta_{n}^{r-2}},
$$

as done by Saulis and Statulevičius in [SS91] or by the authors of this paper in [FMN16]. In particular, it was proved in [FMN16, Chapter 5], that under slight additional assumptions on the second and third cumulants, we have the following: the sequence $\left(X_{n}\right)_{n \in \mathbb{N}}$ defined in Lemma 29 converges in the modGaussian sense with parameters $\left(t_{n}\right)_{n \in \mathbb{N}}$.

\subsection{Dependency graphs}

In this paragraph, we will see that the uniform bounds on cumulants are satisfied for sums $S_{n}=$ $\sum_{i=1}^{n} A_{i, n}$ of dependent random variables with specific dependency structure.

More precisely, if $\left(A_{v}\right)_{v \in V}$ is a family of real valued random variables, we call dependency graph for this family a graph $G=(V, E)$ with the following property: if $V_{1}$ and $V_{2}$ are two disjoint subsets of $V$ with no edge $e \in E$ joining a vertex $v_{1} \in V_{1}$ to a vertex $v_{2} \in V_{2}$, then $\left(A_{v}\right)_{v \in V_{1}}$ and $\left(A_{v}\right)_{v \in V_{2}}$ are independent random vectors. For instance, let $\left(A_{1}, \ldots, A_{7}\right)$ be a family of random variables with dependency graph drawn on Figure 6 . Then the vectors $\left(A_{1}, A_{2}, A_{3}, A_{4}, A_{5}\right)$ and $\left(A_{6}, A_{7}\right)$ corresponding to different connected components must be independent. Moreover, note that $\left(A_{1}, A_{2}\right)$ and $\left(A_{4}, A_{5}\right)$ must be independent as well: although they are in the same connected component of the graph $G$, they are not directly connected by an edge $e \in E$.

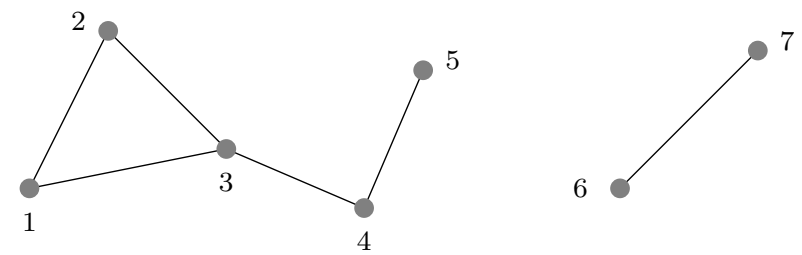

Fig. 6 A dependency graph for 7 real-valued random variables.

Theorem 34 (Féray-Méliot-Nikeghbali, see [FMN16]). Let $\left(A_{v}\right)_{v \in V}$ be a family of random variables, with $\left|A_{v}\right| \leq A$ a.s., for all $v \in V$. We suppose that $G=(V, E)$ is a dependency graph for the family and denote

- $N=\frac{\sum_{v \in V} \mathbb{E}\left|A_{v}\right|}{A} \leq \operatorname{card} V$;

- $D$ the maximum degree of a vertex in $G$ plus one.

If $S=\sum_{v \in V} A_{v}$, then for all $r \geq 1$,

$$
\left|\kappa^{(r)}(S)\right| \leq N r^{r-2}(2 D)^{r-1} A^{r} .
$$


Consider a sequence $\left(S_{n}\right)_{n \in \mathbb{N}}$, where each $S_{n}$ writes as $\sum_{v \in V_{n}} A_{v, n}$, with the $A_{v, n}$ uniformly bounded by $A$ (in a lot of examples, the $A_{v, n}$ are indicator variables, so that we can take $A=1$ ). Set

$$
N_{n}=\frac{\sum_{v \in V_{n}} \mathbb{E}\left|A_{v, n}\right|}{A}
$$

and assume that, for each $n,\left(A_{v, n}\right)_{v \in V_{n}}$ has a dependency graph of maximal degree $D_{n}-1$. Then the sequence $\left(S_{n}\right)_{n \in \mathbb{N}}$ admits uniform bounds on cumulants with parameters $\left(D_{n}, N_{n}, A\right)$ and the result of the previous section applies. Note that in this setting we have the bound $\widetilde{\sigma}_{n} \leq A$, so the bound of Corollary 30 holds with the better constant 52.52 .

Remark 35. The parameter $D$ is equal to the maximal number of neighbors of a vertex $v \in V$, plus 1 . In the following, we shall simply call $D$ the maximal degree, being understood that one always has to add 1. Another way to deal with this convention is to think of dependency graphs as having one loop at each vertex.

Example 36. The following example, though quite artificial, shows that one can construct families of

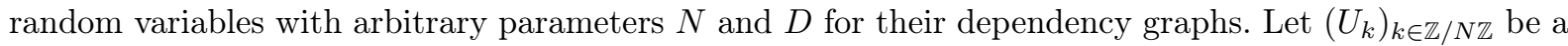
family of independent Bernoulli random variables with $\mathbb{P}\left[U_{k}=1\right]=1-\mathbb{P}\left[U_{k}=0\right]=q$; and for $i \in \mathbb{Z} / N \mathbb{Z}$,

$$
A_{i}=2\left(\prod_{k=i+1}^{i+D} U_{k}\right)-1 .
$$

Each $A_{i}$ is a Bernoulli random variable with $\mathbb{P}\left[A_{i}=1\right]=1-\mathbb{P}\left[A_{i}=-1\right]=q^{D}$, which we denote $p(p$ is considered independent of $N$ ). We are interested in the fluctuations of $S=\sum_{i=1}^{N} A_{i}$. Note that the partial sums $\sum_{i=1}^{k \leq N} A_{i}$ correspond to random walks with correlated steps: as $D$ increases, the consecutive steps of the random walk have a higher probability to be in the same direction, and therefore, the variance of the sum $S=\sum_{i=1}^{N} A_{i}$ grows. We refer to Figure 7, where three such random walks are drawn, with parameters $p=\frac{1}{2}, N=1000$ and $D \in\{5,15,30\}$.

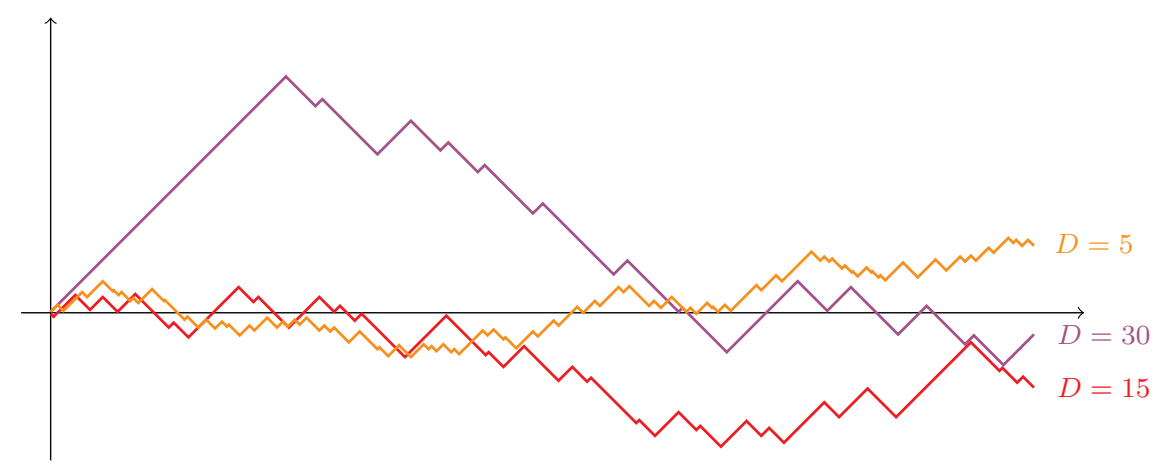

Fig. 7 Random walks with 1000 steps and correlation lengths $D=5, D=15$ and $D=30$.

If $d(i, j) \geq D$ in $\mathbb{Z} / N \mathbb{Z}$, then $A_{i}$ and $A_{j}$ do not involve any common variable $U_{k}$, and they are independent. It follows that if $G$ is the graph with vertex set $\mathbb{Z} / N \mathbb{Z}$ and with an edge $e$ between $i$ and $j$ if $d(i, j) \leq D$, then $G$ is a dependency graph for the $A_{i}$ 's. This graph has $N$ vertices, and maximal degree $2 D-1$. Moreover, one can compute exactly the expectation and the variance of $S=\sum_{i=1}^{N} A_{i}$ : 


$$
\begin{aligned}
\mathbb{E}[S] & =N(2 p-1) \\
\operatorname{Var}(S) & =4 \sum_{i, j=1}^{N} \operatorname{cov}\left(U_{i+1} \cdots U_{i+D}, U_{j+1} \cdots U_{j+N}\right) \\
& =4 \sum_{i, j=1}^{N}\left(q^{D+\min (D, d(i, j))}-p^{2}\right)=4 N p \sum_{j=-(D-1)}^{D-1}\left(q^{|j|}-p\right) \\
& =4 N p\left(\frac{1+p^{\frac{1}{D}}-2 p}{1-p^{\frac{1}{D}}}-(2 D-1) p\right) .
\end{aligned}
$$

If $N$ and $D$ go to infinity with $D=o(N)$, then $q=p^{\frac{1}{D}}=1+\frac{\log p}{D}+O\left(\frac{1}{D^{2}}\right)$, and

$$
\begin{aligned}
\mathbb{E}[S] & =N(2 p-1) ; \\
\operatorname{Var}(S) & =8 N(D+O(1)) p\left(\frac{1-p}{-\log p}-p\right) .
\end{aligned}
$$

So, one can apply Corollary 30 to the sum $S$, and one obtains:

$$
d_{\text {Kol }}\left(\frac{S-\mathbb{E}[S]}{\sqrt{\operatorname{Var}(S)}}, \mathcal{N}_{\mathbb{R}}(0,1)\right) \leq \frac{6}{\left(p\left(\frac{1-p}{-\log p}-p\right)\right)^{3 / 2}} \sqrt{\frac{D}{N}} .
$$

Example 3\%. Fix $p \in(0,1)$, and consider a random Erdös-Rényi graph $G=G_{n}=G(n, p)$, which means that one keeps at random each edge of the complete graph $K_{n}$ with probability $p$, independently from every other edge. Note that we only consider the case of fixed $p$ here; for $p \rightarrow 0$, we would get rather weak bounds, see [FMN16, Section 10.3.3] for a discussion on bounds on cumulants in this framework.

Let $H=\left(V_{H}, E_{H}\right)$ and $G=\left(V_{G}, E_{G}\right)$ be two graphs. The number of copies of $H$ in $G$ is the number of injections $i: V_{H} \rightarrow V_{G}$ such that, if $\left(h_{1}, h_{2}\right) \in E_{H}$, then $\left(i\left(h_{1}\right), i\left(h_{2}\right)\right) \in E_{G}$. In random graph theory, this is called the subgraph count statistics; we denote it by $I(H, G)$. We refer to Figure 8 for an example, where $H=K_{3}$ is the triangle and $G$ is a random Erdös-Rényi graph of parameters $n=30$ and $p=\frac{1}{10}$.

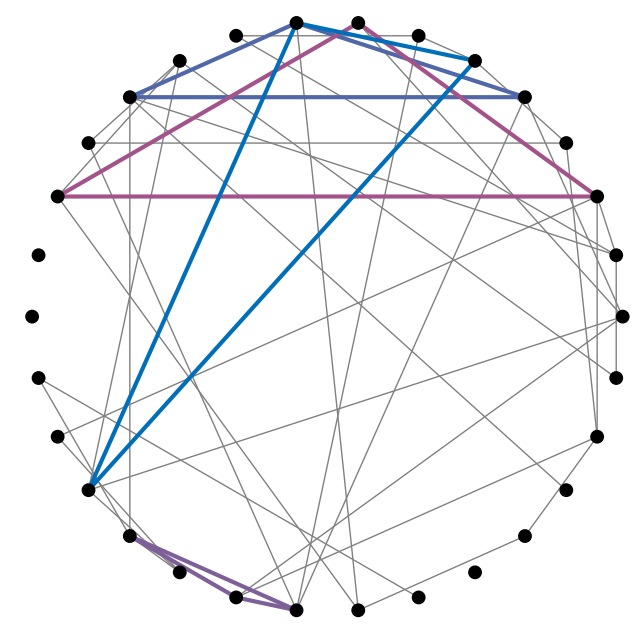

Fig. 8 Count of triangles in a random Erdös-Rényi graph of parameters $n=30$ and $p=0.1$. Here, there are $4 \times 3 !=24$ ways to embed a triangle in the graph.

One can always write $I(H, G)$ as a sum of dependent random variables. Identify $V_{H}$ with $\llbracket 1, k \rrbracket$ and $V_{G}$ with $\llbracket 1, n \rrbracket$, and denote $\mathfrak{A}(n, k)$ the set of arrangements $\left(a_{1}, \ldots, a_{k}\right)$ of size $k$ in $\llbracket 1, n \rrbracket$. Given such 
an arrangement, the induced subgraph $G\left[a_{1}, \ldots, a_{k}\right]$ is the graph with vertex set $\llbracket 1, k \rrbracket$, and with an edge between $i$ and $j$ if $\left(a_{i}, a_{j}\right) \in E_{G}$. Then,

$$
I(H, G)=\sum_{A \in \mathfrak{A}(n, k)} I_{A}(H, G)
$$

where $I_{A}(H, G)=1$ if $H \subset G[A]$, and 0 otherwise.

A dependency graph for the random variables $I_{A}\left(H, G_{n}\right)$ has vertex set $\mathfrak{A}(n, k)$ of cardinality $N_{n}=$ $n^{\downarrow k}=n(n-1)(n-2) \cdots(n-k+1)$, and an edge between two arrangements $\left(a_{1}, \ldots, a_{k}\right)$ and $\left(b_{1}, \ldots, b_{k}\right)$ if they share at least two points (otherwise, the random variables $I_{A}\left(H, G_{n}\right)$ and $I_{B}\left(H, G_{n}\right)$ involve disjoint sets of edges and are therefore independent). As a consequence, the maximal degree of the graph is smaller than

$$
D_{n}=\left(\left(\begin{array}{l}
k \\
2
\end{array}\right)^{2} 2(n-2)(n-3) \cdots(n-k+1)\right),
$$

and of order $n^{k-2}$. Therefore, $\frac{D_{n}}{N_{n}} \leq \frac{2\left(\begin{array}{c}k \\ 2\end{array}\right)^{2}}{n(n-1)}=O\left(\frac{1}{n^{2}}\right)$, and on the other hand, if $h$ is the number of edges of $H$, one can compute the asymptotics of the expectation and of the variance of $I\left(H, G_{n}\right)$ :

$$
\begin{aligned}
\mathbb{E}\left[I\left(H, G_{n}\right)\right] & =n^{\downarrow k} p^{h} ; \\
\operatorname{Var}\left(I\left(H, G_{n}\right)\right) & =2 h^{2} p^{2 h-1}(1-p) n^{2 k-2}+O\left(n^{2 k-3}\right),
\end{aligned}
$$

see [FMN16, Section 10] for the details of these computations. In particular,

$$
\lim _{n \rightarrow \infty} \frac{\operatorname{Var}\left(S_{n}\right)}{N_{n} D_{n}}=p^{2 h-1}(1-p)\left(\frac{h}{\left(\begin{array}{c}
k \\
2
\end{array}\right)}\right)^{2}=\widetilde{\sigma}^{2}>0 .
$$

Thus, using Corollary 30, we get

$$
d_{\text {Kol }}\left(\frac{I\left(H, G_{n}\right)-\mathbb{E}\left[I\left(H, G_{n}\right)\right]}{\sqrt{\operatorname{Var}\left(I\left(H, G_{n}\right)\right)}}, \mathcal{N}_{\mathbb{R}}(0,1)\right) \leq \frac{4.65(k(k-1))^{4}}{p^{3 h}\left(\frac{1}{p}-1\right)^{3 / 2} h^{3}} \frac{1}{n}
$$

for $n$ large enough. For instance, if $T_{n}=I\left(K_{3}, G_{n}\right)$ is the number of triangles in $G_{n}$, then

$$
\begin{aligned}
\mathbb{E}\left[T_{n}\right] & =n^{\downarrow 3} p^{3} \\
\operatorname{Var}\left[T_{n}\right] & =18 n^{\downarrow 4} p^{5}(1-p)+6 n^{\downarrow 3} p^{3}\left(1-p^{3}\right)
\end{aligned}
$$

and

$$
\begin{aligned}
& d_{\text {Kol }}\left(\frac{T_{n}-n^{\downarrow 3} p^{3}}{\sqrt{18 n^{\downarrow 4} p^{5}(1-p)+6 n^{\downarrow 3} p^{3}\left(1-p^{3}\right)}}, \mathcal{N}_{\mathbb{R}}(0,1)\right) \\
& \leq \frac{234}{p^{9}\left(\frac{1}{p}-1\right)^{\frac{3}{2}}} \frac{1}{n}\left(1+O\left(\frac{1}{n}\right)\right) .
\end{aligned}
$$

This result is not new, except maybe the explicit constant. We refer to [BKR89] for an approach of speed of convergence for subgraph counts using Stein's method. More recently, Krokowski, Reichenbachs and Thäle [KRT15] applied Malliavin calculus to the same problem. Our result corresponds to the case where $p$ is constant of their Theorem 1. Similar bounds could be obtained by Stein's method, see [Rin94].

To conclude our presentation of the convergence of sums of bounded random variables with sparse dependency graphs, let us analyse precisely the case of uncorrelated random variables.

Corollary 38. Let $S_{n}=\sum_{i=1}^{N_{n}} A_{i, n}$ be a sum of centered and bounded random variables, that are uncorrelated and with $\mathbb{E}\left[\left(A_{i, n}\right)^{2}\right]=1$ for all $i$. We suppose that the random variables have a dependency graph of parameters $N_{n} \rightarrow+\infty$ and $D_{n}$. 
1. If $D_{n}=O\left(\left(N_{n}\right)^{1 / 2-\varepsilon}\right)$ for $\varepsilon>0$, then $Y_{n}=\frac{S_{n}}{\sqrt{N_{n}}}$ converges in law towards the Gaussian distribution.

2. If $D_{n}=o\left(\left(N_{n}\right)^{1 / 4}\right)$, then the Kolmogorov distance between $Y_{n}$ and $\mathcal{N}_{\mathbb{R}}(0,1)$ is a $O\left(\left(D_{n}\right)^{2} /\left(N_{n}\right)^{1 / 2}\right)$.

Proof. It is an immediate consequence of Corollary 30 and Proposition 32, since $S_{n}$ admits uniform control on cumulants (see Theorem 34) and

$$
\operatorname{Var}\left(S_{n}\right)=\sum_{i=1}^{N_{n}} \mathbb{E}\left[\left(A_{i, n}\right)^{2}\right]=N_{n}
$$

\subsection{Unbounded random variables and truncation methods}

A possible generalization regards sums of unbounded random variables. In the following, we develop a truncation method that yields a criterion of asymptotic normality similar to Lyapunov's condition (see [Bil95, Chapter 27]). A small modification of this method would similarly yield a Lindeberg type criterion. Let $S_{n}=\sum_{i=1}^{N_{n}} A_{i, n}$ be a sum of centered random variables, with

$$
\left(\mathbb{E}\left[\left|A_{i, n}\right|^{2+\delta}\right]\right)^{\frac{1}{2+\delta}} \leq A
$$

for some constant $A$ independent of $i$ and $n$, and some $\delta>0$. We suppose as before that the family of random variables $\left(A_{i, n}\right)_{i \in \llbracket 1, N_{n} \rrbracket}$ has a (true) dependency graph $G_{n}$ of parameters $N_{n}$ and $D_{n}$. Note that in this case,

$$
\begin{aligned}
\operatorname{Var}\left(S_{n}\right) & =\sum_{i, j=1}^{N_{n}} \operatorname{cov}\left(A_{i, n}, A_{j, n}\right) \leq \sum_{i=1}^{N_{n}} \sum_{j \sim i}\left\|A_{i, n}\right\|_{2}\left\|A_{j, n}\right\|_{2} \\
& \leq \sum_{i=1}^{N_{n}} \sum_{j \sim i}\left\|A_{i, n}\right\|_{2+\delta}\left\|A_{j, n}\right\|_{2+\delta} \leq A^{2} N_{n} D_{n} .
\end{aligned}
$$

We set

$$
\begin{aligned}
A_{i, n}^{-} & =A_{i, n} 1_{\left|A_{i, n}\right| \leq L_{n}} & ; & A_{i, n}^{+}=A_{i, n} 1_{\left|A_{i, n}\right|>L_{n}} ; \\
S_{n}^{-} & =\sum_{i=1}^{N_{n}} A_{i, n}^{-} & ; & S_{n}^{+}=\sum_{i=1}^{N_{n}} A_{i, n}^{+} ;
\end{aligned}
$$

where $L_{n}$ is a truncation level, to be chosen later. Notice that $G_{n}$ is still a dependency graph for the family of truncated random variables $\left(A_{i, n}^{-}\right)_{i \in \llbracket 1, N_{n} \rrbracket}$. Therefore, we can apply the previously developed machinery (Theorem 34 and Corollary 30 ) to the scaled sum $S_{n}^{-} / L_{n}$. On the other hand, by Markov's inequality,

$$
\begin{aligned}
d_{\mathrm{Kol}}\left(S_{n}, S_{n}^{-}\right) & =\sup _{s \in \mathbb{R}}\left|\mathbb{P}\left[S_{n} \geq s\right]-\mathbb{P}\left[S_{n}^{-} \geq s\right]\right| \leq \mathbb{P}\left[S_{n}^{+}=0\right] \\
& \leq \sum_{i=1}^{N_{n}} \mathbb{P}\left[\left|A_{i, n}\right| \geq L_{n}\right] \leq N_{n}\left(\frac{A}{L_{n}}\right)^{2+\delta} .
\end{aligned}
$$

Combining the two arguments leads to the following result (this replaces the previous assumption of boundedness $\left.\left|A_{i, n}\right| \leq A\right)$.

Theorem 39. Let $\left(S_{n}=\sum_{i=1}^{N_{n}} A_{i, n}\right)_{n \in \mathbb{N}}$ be a sum of centered random variables, with dependency graphs of parameters $N_{n} \rightarrow+\infty$ and $D_{n}$, and with 


$$
\left\|A_{i, n}\right\|_{2+\delta}=\left(\mathbb{E}\left[\left|A_{i, n}\right|^{2+\delta}\right]\right)^{1 /(2+\delta)} \leq A
$$

for all $i, n$ and for some $\delta>0$. We set $Y_{n}=S_{n} / \sqrt{\operatorname{Var}\left(S_{n}\right)}$. Recall that $\left(\widetilde{\sigma}_{n}\right)^{2}=\frac{\operatorname{Var}\left(S_{n}\right)}{N_{n} D_{n}}$.

(U1) Set

$$
V_{n}=\left(\widetilde{\sigma}_{n}\right)^{2}\left(\frac{N_{n}}{D_{n}}\right)^{1 / 3} \frac{1}{\left(N_{n}\right)^{2 /(2+\delta)}}
$$

and suppose that $\lim _{n \rightarrow \infty} V_{n}=+\infty$ (which is only possible for $\delta>4$ ). Then, for $n$ large enough,

$$
d_{\mathrm{Kol}}\left(Y_{n}, \mathcal{N}_{\mathbb{R}}(0,1)\right) \leq 78\left(\frac{A^{2}}{V_{n}}\right)^{\frac{3(\delta+2)}{2(\delta+5)}}=o\left(\frac{1}{V_{n}}\right)
$$

(U2) More generally, for $\varepsilon \in\left(\frac{2}{2+\delta}, 1\right)$, set

$$
W_{n}=\left(\widetilde{\sigma}_{n}\right)^{2}\left(\frac{N_{n}}{D_{n}}\right)^{\varepsilon} \frac{1}{\left(N_{n}\right)^{2 /(2+\delta)}}
$$

and suppose that $\lim _{n \rightarrow \infty} W_{n}=+\infty$. Then, $Y_{n} \rightarrow \mathcal{N}_{\mathbb{R}}(0,1)$.

Remark 40. It should be noticed that if $\delta \rightarrow+\infty$, then one essentially recovers the content of Corollary 30 (which can be applied because of Theorem 34). On the other hand, the inequality $\delta>4$ amounts to the existence of bounded moments of order strictly higher than 6 for the random variables $A_{i, n}$. In practice, one can for instance ask for bounded moments of order 7 (i.e. $\delta=5)$, in which case the first condition (U1) reads

$$
\lim _{n \rightarrow \infty} \frac{\operatorname{Var}\left(S_{n}\right)}{D_{n} N_{n}} \frac{\left(N_{n}\right)^{1 / 9}}{\left(D_{n}\right)^{1 / 3}}=+\infty .
$$

Moreover, we will see in the proof of Theorem 39 that in this setting $(\delta=5)$, the constant 78 can be improved to 39 , so that, for $n$ large enough:

$$
d_{\mathrm{Kol}}\left(Y_{n}, \mathcal{N}_{\mathbb{R}}(0,1)\right) \leq 39\left(\frac{A^{2}}{V_{n}}\right)^{\frac{21}{20}}
$$

Proof. We write as usual $Y_{n}=\frac{S_{n}}{\sqrt{\operatorname{Var}\left(S_{n}\right)}}$, and $Y_{n}^{-}=\frac{S_{n}^{-}}{\sqrt{\operatorname{Var}\left(S_{n}^{-}\right)}}$. In all cases, we have

$$
\begin{aligned}
& d_{\mathrm{Kol}}\left(Y_{n}, \mathcal{N}_{\mathbb{R}}(0,1)\right) \leq d_{\mathrm{Kol}}\left(S_{n}, S_{n}^{-}\right)+d_{\mathrm{Kol}}\left(Y_{n}^{-}, \sqrt{\frac{\operatorname{Var}\left(S_{n}\right)}{\operatorname{Var}\left(S_{n}^{-}\right)}} \mathcal{N}_{\mathbb{R}}(0,1)\right) \\
& \leq N_{n}\left(\frac{A}{L_{n}}\right)^{2+\delta}+d_{\mathrm{Kol}}\left(Y_{n}^{-}, \mathcal{N}_{\mathbb{R}}(0,1)\right)+d_{\mathrm{Kol}}\left(\mathcal{N}_{\mathbb{R}}(0,1), \mathcal{N}_{\mathbb{R}}\left(0, \frac{\operatorname{Var}\left(S_{n}\right)}{\operatorname{Var}\left(S_{n}^{-}\right)}\right)\right)
\end{aligned}
$$

by using the invariance of the Kolmogorov distance with respect to multiplication of random variables by a positive constant. In the sequel, we denote $a, b$ and $c$ the three terms on the second line of the inequality. The Kolmogorov distance between two Gaussian distributions is

$$
\begin{aligned}
d_{\mathrm{Kol}}\left(\mathcal{N}_{\mathbb{R}}(0,1), \mathcal{N}_{\mathbb{R}}\left(0, \lambda^{2}\right)\right) & =\frac{1}{\sqrt{2 \pi}} \sup _{s \in \mathbb{R}_{+}}\left(\int_{s}^{\lambda s} \mathrm{e}^{-\frac{u^{2}}{2}} d u\right) \\
& \leq \frac{\lambda-1}{\sqrt{2 \pi}} \sup _{s \in \mathbb{R}_{+}}\left(s \mathrm{e}^{-\frac{s^{2}}{2}}\right)=\sqrt{\frac{1}{2 \pi \mathrm{e}}}|\lambda-1|
\end{aligned}
$$

if $\lambda \geq 1$. One gets the same result if $\lambda \leq 1$, hence, 


$$
\begin{aligned}
d_{\text {Kol }}\left(\mathcal{N}_{\mathbb{R}}(0,1), \mathcal{N}_{\mathbb{R}}\left(0, \frac{\operatorname{Var}\left(S_{n}\right)}{\operatorname{Var}\left(S_{n}^{-}\right)}\right)\right) & =d_{\text {Kol }}\left(\mathcal{N}_{\mathbb{R}}(0,1), \mathcal{N}_{\mathbb{R}}\left(0, \frac{\operatorname{Var}\left(S_{n}^{-}\right)}{\operatorname{Var}\left(S_{n}\right)}\right)\right) \\
& \leq \sqrt{\frac{1}{2 \pi \mathrm{e}}}\left|\sqrt{\frac{\operatorname{Var}\left(S_{n}^{-}\right)}{\operatorname{Var}\left(S_{n}\right)}}-1\right| \\
& \leq \sqrt{\frac{1}{2 \pi \mathrm{e}}} \frac{\left|\operatorname{Var}\left(S_{n}^{-}\right)-\operatorname{Var}\left(S_{n}\right)\right|}{\operatorname{Var}\left(S_{n}\right)} .
\end{aligned}
$$

To evaluate the difference between the variances, notice that

$$
\begin{aligned}
\operatorname{Var}\left(S_{n}^{-}\right) & =\operatorname{Var}\left(S_{n}-\sum_{i=1}^{N_{n}} A_{i, n}^{+}\right) \\
& =\operatorname{Var}\left(S_{n}\right)-2 \sum_{i, j=1}^{N_{n}} \operatorname{Cov}\left(A_{i, n}^{+}, A_{j, n}\right)+\sum_{i, j=1}^{N_{n}} \operatorname{Cov}\left(A_{i, n}^{+}, A_{j, n}^{+}\right)
\end{aligned}
$$

If $j$ is not connected to $i$ in $G_{n}$, or equal to $i$, then $A_{i, n}$ and $A_{j, n}$ are independent, hence, $\operatorname{Cov}\left(A_{i, n}^{+}, A_{j, n}\right)=$ 0 . Otherwise, using Hölder and Bienaymé-Chebyshev inequalities,

$$
\begin{aligned}
\left|\operatorname{Cov}\left(A_{i, n}^{+}, A_{j, n}\right)\right| & \leq \sqrt{\mathbb{E}\left[\left(A_{i, n}\right)^{2} 1_{\left|A_{i, n}\right| \geq L_{n}}\right] \mathbb{E}\left[\left(A_{j, n}\right)^{2} 1_{\left|A_{i, n}\right| \geq L_{n}}\right]} \\
& \leq \sqrt{\mathbb{E}\left[\left(A_{i, n}\right)^{2+\delta}\right]^{\frac{2}{2+\delta}} \mathbb{P}\left[\left|A_{i, n}\right| \geq L_{n}\right]^{\frac{2 \delta}{2+\delta}} \mathbb{E}\left[\left(A_{j, n}\right)^{2+\delta}\right]^{\frac{2}{2+\delta}}} \\
& \leq A^{2}\left(\frac{A}{L_{n}}\right)^{\delta} .
\end{aligned}
$$

Similarly,

$$
\begin{aligned}
\left|\operatorname{Cov}\left(A_{i, n}^{+}, A_{j, n}^{+}\right)\right| & \leq \mathbb{E}\left[\left|A_{i, n}^{+} A_{j, n}^{+}\right|\right]+\mathbb{E}\left[\left|A_{i, n}^{+}\right|\right] \mathbb{E}\left[\left|A_{j, n}^{+}\right|\right] \\
& \leq A^{2}\left(\left(\frac{A}{L_{n}}\right)^{\delta}+\left(\frac{A}{L_{n}}\right)^{2+2 \delta}\right)
\end{aligned}
$$

hence, assuming that the level of truncation $L_{n}$ is larger than $A$,

$$
\frac{\left|\operatorname{Var}\left(S_{n}\right)^{-}-\operatorname{Var}\left(S_{n}\right)\right|}{\operatorname{Var}\left(S_{n}\right)} \leq \frac{N_{n} D_{n}}{\operatorname{Var}\left(S_{n}\right)} 3 A^{2}\left(\frac{A}{L_{n}}\right)^{\delta} .
$$

Let us now place ourselves in the setting of Hypothesis (U1); we set

$$
V_{n}=\frac{\operatorname{Var}\left(S_{n}\right)}{N_{n} D_{n}}\left(\frac{N_{n}}{D_{n}}\right)^{1 / 3} \frac{1}{\left(N_{n}\right)^{2 /(2+\delta)}}
$$

Suppose that $L_{n}=K_{n}\left(N_{n}\right)^{\frac{1}{2+\delta}}$, with $K_{n}$ going to infinity. We then have $a \leq \frac{A^{2+\delta}}{\left(K_{n}\right)^{2+\delta}}$, and on the other hand,

$$
\begin{aligned}
\left|\frac{\operatorname{Var}\left(S_{n}^{-}\right)}{\operatorname{Var}\left(S_{n}\right)}-1\right| & \leq 3 A^{2+\delta}\left(\frac{N_{n} D_{n}}{\operatorname{Var}\left(S_{n}\right)} \frac{1}{\left(N_{n}\right)^{\delta /(2+\delta)}} \frac{1}{\left(K_{n}\right)^{\delta}}\right) \\
& \leq 3 A^{2+\delta}\left(\frac{1}{V_{n}\left(D_{n}\right)^{1 / 3}\left(N_{n}\right)^{2 / 3}\left(K_{n}\right)^{\delta}}\right) \rightarrow 0
\end{aligned}
$$

since by hypothesis, $\lim _{n \rightarrow \infty} \frac{1}{V_{n}}=0$. So, 


$$
c \leq \frac{3 A^{2+\delta}}{\sqrt{2 \pi \mathrm{e}}} \frac{1}{V_{n}\left(D_{n}\right)^{1 / 3}\left(N_{n}\right)^{2 / 3}\left(K_{n}\right)^{\delta}} \leq \frac{3 A^{2+\delta}}{\sqrt{2 \pi \mathrm{e}}} \frac{1}{V_{n}\left(K_{n}\right)^{\delta}} .
$$

Now, the sequence $\left(S_{n}^{-} / L_{n}\right)_{n \in \mathbb{N}}$ is a sequence of sums of centered random variables all bounded by 1 , and to apply Corollary 30 to this sequence, we need

$$
\lim _{n \rightarrow \infty} \frac{\operatorname{Var}\left(S_{n}^{-}\right)}{N_{n} D_{n}}\left(\frac{N_{n}}{D_{n}}\right)^{1 / 3} \frac{1}{\left(L_{n}\right)^{2}}=+\infty
$$

However, the previous computation shows that one can replace $\operatorname{Var}\left(S_{n}\right)$ by $\operatorname{Var}\left(S_{n}^{-}\right)$in this expression without changing the asymptotic behavior, so

$$
\lim _{n \rightarrow \infty} \frac{\operatorname{Var}\left(S_{n}^{-}\right)}{N_{n} D_{n}}\left(\frac{N_{n}}{D_{n}}\right)^{1 / 3} \frac{1}{\left(L_{n}\right)^{2}}=\lim _{n \rightarrow \infty} \frac{V_{n}}{\left(K_{n}\right)^{2}},
$$

which is $+\infty$ if $\left(K_{n}\right)^{2}$ is not growing too fast to $+\infty$ (in comparison to the sequence $V_{n}$ ). Then, by Corollary 30,

$$
b=d_{\mathrm{Kol}}\left(Y_{n}^{-}, \mathcal{N}_{\mathbb{R}}(0,1)\right) \leq 77 \frac{\left(K_{n}\right)^{3}}{\left(V_{n}\right)^{3 / 2}}
$$

for $n$ large enough. Set $K_{n}=B\left(V_{n}\right)^{\frac{3}{2(5+\delta)}}$. Then,

$$
\begin{aligned}
d_{\mathrm{Kol}}\left(Y_{n}, \mathcal{N}_{\mathbb{R}}(0,1)\right) & \leq a+b+c \leq\left(\frac{A^{2+\delta}}{B^{2+\delta}}+77 B^{3}+o(1)\right)\left(\frac{1}{V_{n}}\right)^{\frac{3(2+\delta)}{2(5+\delta)}} \\
& \leq\left(\left(\frac{231}{2+\delta}\right)^{\frac{2+\delta}{5+\delta}}+77\left(\frac{2+\delta}{231}\right)^{\frac{3}{5+\delta}}\right)\left(\frac{A^{2}}{V_{n}}\right)^{\frac{3(2+\delta)}{2(5+\delta)}}
\end{aligned}
$$

for $n$ large enough, and by choosing $B$ in an optimal way. The term in parenthesis is maximal when $\delta=229$, and is then equal to 78 . This ends the proof of (U1), and one gets a better constant smaller than 39 when $\delta=5$.

Under the Hypothesis (U2), we set

$$
W_{n}=\frac{\operatorname{Var}\left(S_{n}\right)}{N_{n} D_{n}}\left(\frac{N_{n}}{D_{n}}\right)^{\varepsilon} \frac{1}{\left(N_{n}\right)^{2 /(2+\delta)}} .
$$

In order to prove the convergence in law $Y_{n} \rightarrow \mathcal{N}_{\mathbb{R}}(0,1)$, it suffices to have:

- $S_{n}-S_{n}^{-}=S_{n}^{+}$that converges in probability to 0 . This happens as soon as the level $L_{n}$ is $K_{n}\left(N_{n}\right)^{1 /(2+\delta)}$ with $K_{n} \rightarrow+\infty$.

- $\frac{\left|\operatorname{Var}\left(S_{n}\right)-\operatorname{Var}\left(S_{n}^{-}\right)\right|}{\operatorname{Var}\left(S_{n}\right)} \rightarrow 0$. With $L_{n}=K_{n}\left(N_{n}\right)^{1 /(2+\delta)}$, the previous computations show that this quantity is a

$$
O\left(\frac{1}{W_{n}\left(N_{n}\right)^{1-\varepsilon}\left(D_{n}\right)^{\varepsilon}\left(K_{n}\right)^{\delta}}\right)
$$

which goes to 0 .

- and by Theorem 32,

$$
\frac{\operatorname{Var}\left(S_{n}\right)}{N_{n} D_{n}}\left(\frac{N_{n}}{D_{n}}\right)^{\varepsilon} \frac{1}{\left(L_{n}\right)^{2}} \rightarrow+\infty .
$$

This follows from the Hypothesis (U2) if $K_{n}$ is chosen to grow sufficiently slow.

Thus, the second part of Theorem 39 is proven.

Example 41. Let $\left(X_{i}\right)_{i \in \llbracket 1, N \rrbracket}$ be a centered Gaussian vector with $\mathbb{E}\left[\left(X_{i}\right)^{2}\right]=1$ for any $i$, and with the covariance matrix $\left(\operatorname{Cov}\left(X_{i}, X_{j}\right)\right)_{1 \leq i, j \leq N}$ that is sparse in the following sense: for any $i$, the set of indices 
$j$ such that $\operatorname{Cov}\left(X_{i}, X_{j}\right) \neq 0$ has cardinality smaller than $D$. We set $A_{i}=\exp \left(X_{i}\right)$; the random variables $A_{i}$ follow the log-normal distribution of density

$$
\frac{1}{\sqrt{2 \pi}} \frac{1}{u^{1+\frac{\log u}{2}}} 1_{u>0} d u
$$

and they have moments of all order: $\mathbb{E}\left[\left(A_{i}\right)^{k}\right]=\mathbb{E}\left[\mathrm{e}^{k X_{i}}\right]=\mathrm{e}^{\frac{k^{2}}{2}}$.

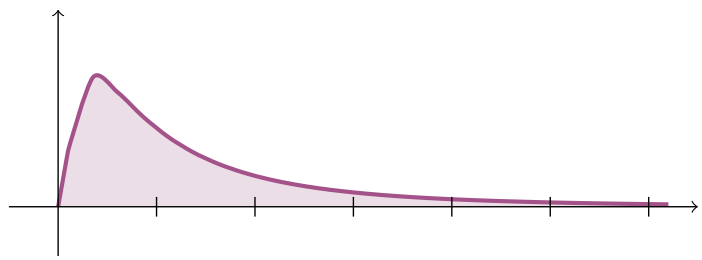

Fig. 9 The density of the log-normal distribution.

The variables $A_{i}$ have the same dependency graph as the variables $X_{i}$. Moreover, if $\rho_{i j}=\operatorname{Cov}\left(X_{i}, X_{j}\right)$, then the covariance of two variables $A_{i}$ and $A_{j}$ is e $\left(\mathrm{e}^{\rho_{i j}}-1\right)$. Using moments of order $2+\delta$, we see that if

$$
\begin{aligned}
Y_{N} & =\frac{\sum_{i=1}^{N}\left(A_{i}-\mathrm{e}^{\frac{1}{2}}\right)}{\sqrt{\mathrm{e} \sum_{1 \leq i, j \leq N}\left(\mathrm{e}^{\rho_{i j}}-1\right)}} \\
V_{N, \delta} & =\frac{\mathrm{e} \sum_{1 \leq i, j \leq N}\left(\mathrm{e}^{\rho_{i j}}-1\right)}{N D} \frac{N^{\frac{1}{3}-\frac{2}{2+\delta}}}{D^{\frac{1}{3}}} \rightarrow+\infty,
\end{aligned}
$$

then $d_{\mathrm{Kol}}\left(Y_{N}, \mathcal{N}_{\mathbb{R}}(0,1)\right) \leq 78\left(\frac{\mathrm{e}^{\delta+2}}{V_{N, \delta}}\right)^{\frac{3(\delta+2)}{2(\delta+5)}}$ for $N$ large enough.

To make this result more explicit, let us consider the following dependency structure for the Gaussian vector $X=\left(X_{i}\right)_{i \in \llbracket 1, N \rrbracket}$ :

$$
\operatorname{Cov}(X)=\left(\begin{array}{cccc}
1 & * & \cdots & * \\
* & 1 & \ddots & \vdots \\
\vdots & \ddots & \ddots & * \\
* & \cdots & * & 1
\end{array}\right)
$$

where the non-diagonal terms $*$ are all smaller than $\frac{\rho}{D}$ in absolute value, and with less than $D$ non-zero terms on each row or column. When $\rho \in[0,1)$, the matrix is diagonally dominant, hence positive-definite, so there exists indeed a Gaussian vector $X$ with these covariances. We then have

$$
V_{N, \delta} \geq \mathrm{e}\left(1-D\left(\mathrm{e}^{\frac{\rho}{D}}-1\right)\right) \frac{N^{\frac{1}{3}-\frac{2}{2+\delta}}}{D^{\frac{4}{3}}},
$$

so if $1 \ll D \ll N^{\frac{1}{4}-\varepsilon}$, then one can apply Theorem 39 to get

$$
d_{\mathrm{Kol}}\left(Y_{N}, \mathcal{N}_{\mathbb{R}}(0,1)\right) \leq 78\left(\frac{\mathrm{e}^{\frac{3}{2 \varepsilon}-1}}{1-\rho}\right)^{\frac{3}{2}}\left(\frac{D}{N^{\frac{1}{4}-\varepsilon}}\right)^{\frac{2}{2 \varepsilon+1}}
$$

for $N$ large enough. Moreover, as soon as $1 \ll D \ll N^{\frac{1}{2}-\varepsilon}, Y_{N} \rightarrow \mathcal{N}_{\mathbb{R}}(0,1)$. 


\section{Ising model and Markov chains}

In this section, we present examples of random variables that admit uniform bounds on cumulants, which do not come from dependency graphs. Their structure is nevertheless not so different since the variables that we consider write as sums of random variables that are weakly dependent. The technique to prove uniform bounds on cumulants relies then on the notion of uniform weighted dependency graph, which generalizes the notion of standard dependency graph (see Proposition 44).

\subsection{Weighted graphs and spanning trees}

An edge-weighted graph $G$, or weighted graph for short, is a graph $G$ in which each edge $e$ is assigned a weight $w_{G}(e)$. Here we restrict ourselves to weights $w_{G}(e)$ with $w_{G}(e) \in \mathbb{R}_{+}$. Edges not in the graph can be thought of as edges of weight 0 , all our definitions being consistent with this convention.

If $B$ is a multiset of vertices of $G$, we can consider the graph $G[B]$ induced by $G$ on $B$ and defined as follows: the vertices of $G[B]$ correspond to elements of $B$ (if $B$ contains an element with multiplicity $m$, then $m$ vertices correspond to this element), and there is an edge between two vertices if the corresponding vertices of $G$ are equal or connected by an edge in $G$. This new graph has a natural weighted graph structure: put on each edge of $G[B]$ the weight of the corresponding edge in $G$ (if the edge connects two copies of the same vertex of $G$, we put weight 1 ).

Definition 42. A spanning tree of a graph $G=(V, E)$ is a subset $E^{\prime}$ of $E$ such that $\left(V, E^{\prime}\right)$ is a tree. In other words, it is a subgraph of $G$ that is a tree and covers all vertices.

The set of spanning trees of $T$ is denoted $\operatorname{ST}(G)$. If $G$ is a weighted graph, we say that the weight $w(T)$ of a spanning tree of $G$ is defined as the product of the weights of the edges in $T$.
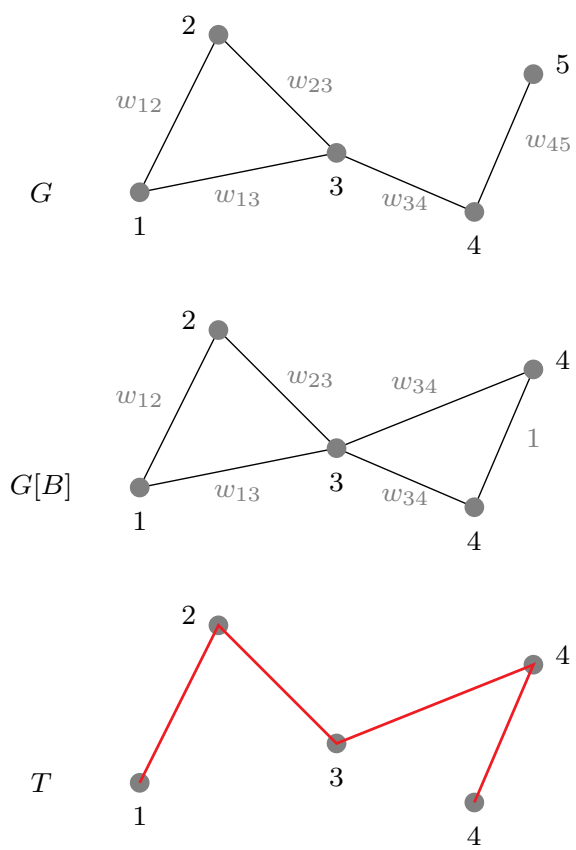

6

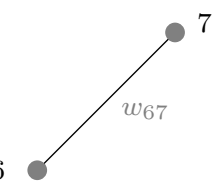

Fig. 10 A weighted dependency graph $G$ for 7 random variables; the induced graph $G[B]$ with $B=\{1,2,3,4,4\}$; and a spanning tree $T$ of $G[B]$, with $w(T)=w_{12} w_{23} w_{34}$. 


\subsection{Uniform weighted dependency graphs}

If $A_{1}, \ldots, A_{r}$ are real-valued random variables, there is a notion of joint cumulant that generalize the cumulants of Section 4:

$$
\kappa\left(A_{1}, A_{2}, \ldots, A_{r}\right)=\left[z_{1} z_{2} \cdots z_{r}\right]\left(\log \mathbb{E}\left[\mathrm{e}^{z_{1} A_{1}+z_{2} A_{2}+\cdots+z_{r} A_{r}}\right]\right) .
$$

The joint cumulants are multilinear and symmetric functionals of $A_{1}, \ldots, A_{r}$. On the other hand,

$$
\kappa^{(r)}(X)=\kappa(X, X, \ldots, X)
$$

with $r$ occurrences of $X$ in the right-hand side. In particular, if $S=\sum_{v \in V} A_{v}$ is a sum of random variables, then

$$
\kappa^{(r)}(S)=\sum_{v_{1}, \ldots, v_{r} \in V} \kappa\left(A_{v_{1}}, A_{v_{2}}, \ldots, A_{v_{r}}\right)
$$

Definition 43. Let $\left(A_{v}\right)_{v \in V}$ be a family of random variables defined on the same probability space. A weighted graph $G=\left(V, E, w_{G}\right)$ is a $C$-uniform weighted dependency graph for $\left(A_{v}\right)_{v \in V}$ if, for any multiset $B=\left\{v_{1}, \ldots, v_{r}\right\}$ of elements of $V$, one has

$$
\left|\kappa\left(A_{v}, v \in B\right)\right| \leq C^{|B|} \sum_{T \in \mathrm{ST}(G[B])} w(T) .
$$

Proposition 44. Let $\left(A_{v}\right)_{v \in V}$ be a finite family of random variables with a $C$-uniform weighted dependency graph $G$. Assume that $G$ has $N=|V|$ vertices, and maximal weighted degree $D-1$, that is:

$$
\forall v \in V, \sum_{\substack{v^{\prime}: v^{\prime} \neq v \\\left\{v, v^{\prime}\right\} \in E_{G}}} w_{G}\left(\left\{v, v^{\prime}\right\}\right) \leq D-1 .
$$

Then, for $r \geq 1$,

$$
\left|\kappa^{(r)}\left(\sum_{v \in V} A_{v}\right)\right| \leq N r^{r-2} D^{r-1} C^{r} .
$$

Consider a sequence $\left(S_{n}\right)_{n \in \mathbb{N}}$, where each $S_{n}$ writes as $\sum_{v \in V_{n}} A_{v, n}$. Set $N_{n}=\left|V_{n}\right|$ and assume that, for each $n,\left(A_{v}\right)_{v \in V_{n}}$ has $C$-uniform weighted dependency graph of maximal degree $D_{n}-1$ (by assumption, $C$ does not depend on $n)$. Then the sequence $\left(S_{n}\right)$ admits uniform bounds on cumulants with parameters $\left(\frac{D_{n}}{2}, N_{n}, C\right)$ and the results of Section 4 , in particular Corollary 30, apply.

Proof. By multilinearity and definition of a uniform weighted dependency graph, we have

$$
\left|\kappa_{r}\left(\sum_{v \in V} A_{v}\right)\right| \leq C^{r} \sum_{v_{1}, \ldots, v_{r}} \sum_{T \in \operatorname{ST}\left(G\left[v_{1}, \ldots, v_{r}\right]\right)} w(T) .
$$

By possibly adding edges of weight 0 , we may assume that $G\left[v_{1}, \ldots, v_{r}\right]$ is always the complete graph $K_{r}$ so that $\mathrm{ST}\left(G\left[v_{1}, \ldots, v_{r}\right]\right) \simeq \mathrm{ST}\left(K_{r}\right)$ as sets. The weight of a tree $T$ however depends on $v_{1}, \cdots, v_{r}$, namely

$$
w(T)=\prod_{\{i, j\} \in T} w_{G}\left(\left\{v_{i}, v_{j}\right\}\right),
$$

where $w_{G}\left(\left\{v_{i}, v_{j}\right\}\right)$ is the weight of the edge $\left\{v_{i}, v_{j}\right\}$ in $G$ (or 1 if $v_{i}=v_{j}$ ).

With this viewpoint, we can exchange the order of summation in (7). We claim that the contribution of a fixed tree $T \in \mathrm{ST}\left(K_{r}\right)$ can then be bounded as follows:

$$
\sum_{v_{1}, \ldots, v_{r}} w(T)=\sum_{v_{1}, \ldots, v_{r}} \prod_{\{i, j\} \in T} w_{G}\left(\left\{v_{i}, v_{j}\right\}\right) \leq N D^{r-1} .
$$


Let us prove this claim by induction on $r$. The case $r=1$ is trivial. Up to renaming the vertices of $T$, we may assume that $r$ is a leaf of $T$ so that $T$ is obtained from a spanning tree $\widetilde{T}$ of $K_{r-1}$ by adding an edge $\left\{i_{0}, r\right\}$ for some $i_{0}<r$. Then

$$
\begin{aligned}
& \sum_{v_{1}, \ldots, v_{r}} \prod_{\{i, j\} \in T} w_{G}\left(\left\{v_{i}, v_{j}\right\}\right) \\
= & \sum_{v_{1}, \ldots, v_{r-1}}\left(\prod_{\{i, j\} \in \widetilde{T}} w_{G}\left(\left\{v_{i}, v_{j}\right\}\right)\right)\left[\sum_{v_{r} \in V} w_{G}\left(\left\{v_{i_{0}}, v_{r}\right\}\right)\right] .
\end{aligned}
$$

The expression in square brackets is by definition smaller than $D$ for all $v_{i_{0}}$ (the sum for $v_{r} \neq v_{i_{0}}$ is smaller than $D-1$ and the term for $v_{r}=v_{i_{0}}$ is 1 ). By induction hypothesis, the sum of the parenthesis is smaller than $N D^{r-2}$. This concludes the proof of (8) by induction. The lemma now follows immediately, since the number of spanning trees of $K_{r}$ is well known to be $r^{r-2}$.

Remark 45. A classical dependency graph with a uniform bound $A$ on all variables $A_{v}$ can be seen as a $C$-uniform weighted dependency graph for $C=2 A$ (all edges have weight 1); see [FMN16, Section 9.4]. In this case, Proposition 44 reduces to Theorem 34. The proof of Proposition 44 given here is a simple adaptation of the second part of the proof of Theorem 34 (see [FMN16, Chapter 9]) to the weighted context. The first, and probably the hardest part of the proof of Theorem 34 consisted in showing that a classical dependency graph is indeed a $C$-uniform weighted dependency graph.

Remark 46. In the case where the set $V$ is a subset of $\mathbb{Z}^{d}$ and the weight function only depends on the distance, the notion of uniform weighted dependency graph coincides with the notion of strong cluster properties, proposed by Duneau, Iagolnitzer and Souillard in [DIS73]. These authors also observed that this implies uniform bounds on cumulants when $D$ is bounded, see [DIS73, Eq. (10)].

Remark 4\%. A weaker notion of weighted dependency graph, where the bound on cumulant is not uniform on $r$, was recently introduced in [Fér16]. This weaker notion only enables to prove central limit theorem, without normality zone or speed of convergence results. However, it seems to have a larger range of applications.

\subsection{Magnetization of the Ising model}

We consider here the nearest-neighbour Ising model on a finite subset $\Lambda$ of $\mathbb{Z}^{d}$ with a quadratic potential, i.e. for a spin configuration $\sigma$ in $\{-1,+1\}^{\Lambda}$, its energy is given by

$$
\mathcal{H}_{\beta, h}^{\Lambda}(w)=-\beta \sum_{\substack{i, j \in \Lambda \\\{i, j\} \in E_{\mathbb{Z}^{d}}}} \sigma_{i} \sigma_{j}-h \sum_{i \in \Lambda} \sigma_{i},
$$

where $E_{\mathbb{Z}^{d}}$ is the set of edges of the lattice $\mathbb{Z}^{d}$ and $h$ and $\beta$ are two real parameters with $\beta>0$. The probability $\mu_{\beta, h, \Lambda}[\sigma]$ of taking a configuration $\sigma$ is then proportional to $\exp \left(-\mathcal{H}_{\beta, h}^{\Lambda}(\sigma)\right)$.

We now want to make $\Lambda$ grow to the whole lattice $\mathbb{Z}^{d}$. It is well known that for $h \neq 0$ or $\beta$ smaller than a critical value $\beta_{c}(d)$ (thus, at high temperature), there is a unique limiting probability measure $\mu_{\beta, h}$ on spin configurations on $\mathbb{Z}^{d}$, see e.g. [FV16, Theorem 3.41]. In the following, we take parameters $(\beta, h)$ in this domain of uniqueness and consider a random spin configuration $\sigma$, whose law is $\mu_{\beta, h}$.

In [DIS74], Duneau, Iagolnitzer and Souillard proved what they call the strong cluster properties for spins in the Ising model for $h \neq 0$ or sufficiently small $\beta$. Their result is actually more general (it holds for other models than the Ising model) but for simplicity, we stick to the Ising model here. Reformulated with the terminology of the present article, we have:

Theorem 48 (Duneau, Iagolnitzer and Souillard, 1974). Fix the dimension $d \geq 1$ and $h \neq 0, \beta>0$. 
1. There exist $C=C(d, \beta, h)$ and $\varepsilon=\varepsilon(d, \beta, h)<1$ such that under the probability measure $\mu_{\beta, h}$, the family $\left\{\sigma_{i}, i \in \mathbb{Z}^{d}\right\}$ has a $C$-uniform weighted dependency graph $G$, where the weight of the edge $\{i, j\}$ in $G$ is $\varepsilon^{\|i-j\|_{1}}$.

2. The same holds for $h=0$ and $\beta$ is sufficiently small (i.e. $\beta<\beta_{1}(d)$, for some $\beta_{1}(d)$ depending on the dimension; this is sometimes refered to as the very high temperature regime).

Note that the maximal weighted degree of this graph is a constant $C^{\prime}<\infty$.

We now consider the magnetization in a finite box $\Delta$ defined as $M_{\Delta}=\sum_{i \in \Delta} \sigma_{i}$. We see $M_{\Delta}$ as a sequence of random variables (indexed by the countably many finite subsets of $\mathbb{Z}^{d}$ ). Restricting the uniform weighted dependency graph above to $\left\{\sigma_{i}, i \in \Delta\right\}$, each $M_{\Delta}$ is the sum of random variables with a $C$-uniform weighted dependency graph and maximal weighted degree at most $C^{\prime}$. Therefore, using Proposition 44, we know that $M_{|\Delta|}$ admits uniform bounds on cumulants with parameters $\left(\frac{C^{\prime}}{2},|\Delta|, C\right)$. Moreover, since all spins are positively correlated by the FKG inequality (see [FV16, Section 3.6]), we have, using translation invariance

$$
\operatorname{Var}\left(M_{\Delta}\right) \geq \sum_{i \in \Delta} \operatorname{Var}\left(\sigma_{i}\right)=\operatorname{Var}\left(\sigma_{0}\right)|\Delta| .
$$

Note that $\operatorname{Var}\left(\sigma_{0}\right)$ is independent of $\Delta$. With the notation of Section 4 , this inequality ensures that $\widetilde{\sigma}_{\Delta}$ is bounded from below by a constant. Applying Corollary 30, we get:

Proposition 49. Fix the dimension $d \geq 1$ and parameters $h \neq 0, \beta>0$. The exists a constant $K=$ $K(d, \beta, h)$ such that, for all subsets $\Delta$ of $\mathbb{Z}^{d}$, we have under $\mu_{\beta, h}$

$$
d_{\text {Kol }}\left(\frac{M_{\Delta}-\mathbb{E}\left[M_{\Delta}\right]}{\sqrt{\operatorname{Var}\left(M_{\Delta}\right)}}, \mathcal{N}_{\mathbb{R}}(0,1)\right) \leq \frac{K}{\sqrt{|\Delta|}} .
$$

The same holds for $h=0$ and $\beta$ sufficiently small (very high temperature).

Remark 50. In this remark, we discuss mod-Gaussian convergence in this setting. Consider a sequence $\Delta_{n}$ of subsets of $\mathbb{Z}^{d}$, tending to $\mathbb{Z}^{d}$ in the Van Hove sense (i.e. the sequence is increasing with union $\mathbb{Z}^{d}$, and the size of the boundary of $\Delta_{n}$ is asymptotic negligible, compared to the size of $\Delta_{n}$ itself). Then it is known from [Ell85, Lemma 5.7.1] that

$$
\lim _{n \rightarrow \infty} \frac{1}{|\Delta|} \operatorname{Var}\left(M_{\Delta}\right)=\sum_{k \in \mathbb{Z}^{d}} \operatorname{Cov}\left(\sigma_{0}, \sigma_{k}\right),
$$

and the right-hand side has a finite value $\widetilde{\sigma}^{2}(\beta, h)$ for parameters $(\beta, h)$ for which Theorem 48 applies. Similarly, we have

$$
\lim _{n \rightarrow \infty} \frac{1}{|\Delta|} \kappa^{(3)}\left(M_{\Delta}\right)=\sum_{k, l \in \mathbb{Z}^{d}} \kappa\left(\sigma_{0}, \sigma_{k}, \sigma_{l}\right)<\infty .
$$

We call $\rho(\beta, h)$ this quantity, and denote $L=\frac{\rho(\beta, h)}{\tilde{\sigma}^{3}(\beta, h)}$. Let us then consider the rescaled variables

$$
X_{n}=\frac{M_{\Delta_{n}}-\mathbb{E}\left[M_{\Delta_{n}}\right]}{\left(\operatorname{Var}\left(M_{\Delta_{n}}\right)\right)^{1 / 3}} .
$$

From [FMN16, Section 5] (with $\alpha_{n}=\operatorname{Var}\left(M_{\Delta}\right)$ and $\beta_{n}=1$ ), we know that $X_{n}$ converges in the complex mod-Gaussian sense with parameters $t_{n}=\left(\operatorname{Var}\left(M_{\Delta_{n}}\right)\right)^{1 / 3}$ and limiting function $\psi(z)=\exp \left(\frac{L z^{3}}{6}\right)$. This mod-Gaussian convergence takes place on the whole complex plane. Using the results of [FMN16], this implies a normality zone for $\left(M_{\Delta}-\mathbb{E}\left[M_{\Delta}\right]\right) / \sqrt{\operatorname{Var}\left(M_{\Delta}\right)}$ of size $o\left(|\Delta|^{1 / 6}\right)$, see Proposition 4.4.1 in loc. cit.; and moderate deviation estimates at the edge of this normality zone, see Proposition 5.2.1.

Remark 51. For $h=0$ and $\beta>\beta_{c}(d)$ (low temperature regime), there is no weighted dependency graph as above. Indeed, this would imply the analycity of the partition function in terms of the magnetic field 
$h$, and the latter is known not to be analytic at $h=0$ for $\beta>\beta_{c}(d)$; see [MM91, Chapter 6, §5] for details.

\subsection{Functionals of Markov chains}

In this section, we consider a discrete time Markov chain $\left(M_{t}\right)_{t \geq 0}$ on a finite state space $\mathfrak{X}$, which is ergodic (irreducible and aperiodic) with invariant measure $\pi$. Its transition matrix is denoted $P$. To simplify the discussion, we shall also assume that the Markov chain is stationary, that is to say that the initial measure (i.e. the law of $M_{0}$ ) is $\pi$; most results have easy corollaries for any initial measure, using the fast mixing of such Markov chains.

Let us consider a sequence $\left(f_{t}\right)_{t \geq 0}$ of functions on $\mathfrak{X}$ that is uniformly bounded by a constant $K$. We set $Y_{t}=f_{t}\left(M_{t}\right)$. We will show that $\left\{Y_{t}\right\}_{t \in \mathbb{N}}$ admits a uniform weighted dependency graph. The proof roughly follows the one of [Fér16, Section 10], where it was proved that it has a (non-uniform) weighted dependency graph, taking extra care of the dependence in the order $r$ of the cumulant in the bounds. Instead of working directly with classical (joint) cumulants, we start by giving a bound for the so-called Boolean cumulants. Classical cumulants are then expressed in terms of Boolean cumulants thanks to a formula of Saulis and Statulevičius [SS91, Lemma 1.1]; see also a recent article of Arizmendi, Hasebe, Lehner and Vargas [AHLV15] (we warn the reader that, in [SS91], Boolean cumulants are called centered moments).

Let $Z_{1}, \ldots, Z_{r}$ be random variables with finite moments defined on the same probability space. By definition, their Boolean (joint) cumulant is

$$
\begin{aligned}
& B^{(r)}\left(Z_{1}, \ldots, Z_{r}\right) \\
& =\sum_{l=0}^{r-1}(-1)^{l} \sum_{1 \leq d_{1}<\cdots<d_{l} \leq r-1} \mathbb{E}\left[Z_{1} \cdots Z_{d_{1}}\right] \mathbb{E}\left[Z_{d_{1}+1} \cdots Z_{d_{2}}\right] \cdots \mathbb{E}\left[Z_{d_{l}+1} \cdots Z_{r}\right] .
\end{aligned}
$$

While not at first sight, this definition is quite similar to the definition of classical (joint) cumulants, replacing the lattice of all set partitions by the lattice of interval set partitions; see [AHLV15, Section 2] for details. Note however that, unlike classical cumulants, Boolean cumulants are not symmetric functionals.

Proposition 52. Let $r \geq 1$. With the above notation, there exists a constant $\theta_{P}$ depending on $P$ with the following property. For any integers $t_{1} \leq t_{2} \leq \cdots \leq t_{r}$, we have

$$
\left|B^{(r)}\left(Y_{t_{1}}, \ldots, Y_{t_{r}}\right)\right| \leq M^{\frac{r-1}{2}} K^{r}\left(\theta_{P}\right)^{t_{r}-t_{1}},
$$

where $M=|\mathfrak{X}|$.

The proof of this bound relies on arguments due to Diaconis, Stroock and Fill, see [DS91, Fil91]. We also refer to [SS91, Section 4.1] for an alternate approach. Given an ergodic transition matrix $P$ on $\mathfrak{X}$ with invariant measure $\pi$, we denote $\widetilde{P}$ the time reversal of $P$, which is the stochastic matrix defined by the equation

$$
\widetilde{P}(x, y)=\frac{\pi(y) P(y, x)}{\pi(x)} .
$$

This new transition matrix is again ergodic, with stationary measure $\pi$. The multiplicative reversiblization of $P$ is the matrix $M(P)=P \widetilde{P}$. It is a stochastic matrix, which is ergodic with stationary measure $\pi$, and with all its eigenvalues that are real and belong to $[0,1]$. Indeed, if $D$ is the diagonal matrix $D=\operatorname{diag}(\pi)$, then $\widetilde{P}=D^{-1} P^{t} D$, and 


$$
\begin{aligned}
\operatorname{Spec}(M(P)) & =\operatorname{Spec}\left(D^{1 / 2} P \widetilde{P} D^{-1 / 2}\right) \\
& =\operatorname{Spec}\left(\left(D^{1 / 2} P D^{-1 / 2}\right)\left(D^{-1 / 2} P^{t} D^{1 / 2}\right)\right) \\
& =\operatorname{Spec}\left(\left(D^{1 / 2} P D^{-1 / 2}\right)\left(D^{1 / 2} P D^{-1 / 2}\right)^{t}\right) .
\end{aligned}
$$

Thus, $M(P)$ has the spectrum of a symmetric positive matrix, so it belongs to $\mathbb{R}_{+}$, and in fact to $[0,1]$ since $M(P)$ is also stochastic. We denote

$$
\left(\theta_{P}\right)^{2}=\max \{|z| \mid z \text { eigenvalue of } M(P), z \neq 1\} .
$$

Notice that if $P$ is reversible, then $\widetilde{P}=P$ and $M(P)=P^{2}$, so in this case

$$
\theta_{P}=\max \{|z| \mid z \text { eigenvalue of } P, z \neq 1\} .
$$

In general, one can think of $\theta_{P}$ as the analogue of the second largest eigenvalue for non-reversible transition matrices. The following result estimates the rate of convergence of the Markov chain associated to $P$ in terms of $\theta$ :

Theorem 53 (Fill, 1991). For any $x \in \mathfrak{X}$,

$$
\begin{aligned}
& \sum_{y \in \mathfrak{X}}\left|P^{t}(x, y)-\pi(y)\right| \leq \frac{\left(\theta_{P}\right)^{t}}{\sqrt{\pi(x)}} \\
& \sum_{y \in \mathfrak{X}} \frac{\left|P^{t}(x, y)-\pi(y)\right|}{\sqrt{\pi(y)}} \leq \sqrt{M} \frac{\left(\theta_{P}\right)^{t}}{\sqrt{\pi(x)}}
\end{aligned}
$$

where $M=|\mathfrak{X}|$.

Proof. For completeness, we reproduce here the discussion of [Fil91, Section 2], which relies on the following identity due to Mihail. If $f$ is a function on $\mathfrak{X}$, we denote $\operatorname{Var}(f)$ its variance under the stationary probability measure $\pi$. We also introduce the Dirichlet form

$$
\mathscr{E}(f, g)=\frac{1}{2} \sum_{x, y \in \mathfrak{X}}(f(x)-f(y))(g(x)-g(y)) \pi(x) M(P)(x, y) .
$$

Then, for any function $f$,

$$
\operatorname{Var}(f)=\operatorname{Var}(\widetilde{P} f)+\mathscr{E}(f, f) .
$$

Indeed, one can assume w.l.o.g. that $\pi(f)=\sum_{x \in \mathfrak{X}} \pi(x) f(x)=0$. If $\langle f \mid g\rangle_{\pi}=\sum_{x \in \mathfrak{X}} \pi(x) f(x) g(x)$, then

$$
\begin{aligned}
\mathscr{E}(f, f) & =\langle f \mid(\mathrm{id}-M(P)) f\rangle_{\pi}=\langle f \mid f\rangle_{\pi}-\langle f \mid P \widetilde{P} f\rangle_{\pi} \\
& =\langle f \mid f\rangle_{\pi}-\langle\widetilde{P} f \mid \widetilde{P} f\rangle_{\pi}=\operatorname{Var}(f)-\operatorname{Var}(\widetilde{P} f)
\end{aligned}
$$

since $\widetilde{P}$ is the adjoint of $P$ for the action on the left of functions and with respect to the scalar product $\langle\cdot \mid \cdot\rangle_{\pi}$. Consider now a Markov chain $\left(X_{t}\right)_{t \in \mathbb{N}}$ on $\mathfrak{X}$ with arbitrary initial distribution $\pi_{0}$, and denote $\pi_{t}=\pi_{0} P^{t}$ the distribution at time $t$. We introduce the quantity

$$
\left(\chi_{t}\right)^{2}=\sum_{y \in \mathfrak{X}} \frac{\left(\pi_{t}(y)-\pi(y)\right)^{2}}{\pi(y)} .
$$

This is the variance of $f_{t}=\frac{\pi_{t}}{\pi}$ with respect to the probability measure $\pi$. By Mihail's identity,

$$
\left(\chi_{t+1}\right)^{2}=\operatorname{Var}\left(f_{t+1}\right)=\operatorname{Var}\left(\widetilde{P} f_{t}\right)=\operatorname{Var}\left(f_{t}\right)-\mathscr{E}\left(f_{t}, f_{t}\right)=\left(\chi_{t}\right)^{2}-\mathscr{E}\left(f_{t}, f_{t}\right) .
$$

By the minimax characterization of eigenvalues of symmetric positive matrices, 


$$
\left(\theta_{P}\right)^{2}=1-\inf \left\{\frac{\mathscr{E}(f, f)}{\operatorname{Var}(f)}, f \text { non-constant }\right\} .
$$

Therefore, $\left(\chi_{t+1}\right)^{2} \leq\left(\theta_{P}\right)^{2}\left(\chi_{t}\right)^{2}$, and $\left(\chi_{t}\right)^{2} \leq\left(\theta_{P}\right)^{2 t}\left(\chi_{0}\right)^{2}$ by induction on $t$. On the other hand, the Cauchy-Schwarz inequality yields

$$
\sum_{y \in \mathfrak{X}}\left|\pi_{t}(y)-\pi(y)\right| \leq \sqrt{\sum_{y \in \mathfrak{X}} \pi(y)} \sqrt{\sum_{y \in \mathfrak{X}} \frac{\left(\pi_{t}(y)-\pi(y)\right)^{2}}{\pi(y)}}=\chi_{t} .
$$

If we choose $\pi_{0}=\delta_{x}$, we finally obtain:

$$
\sum_{y \in \mathfrak{X}}\left|P^{t}(x, y)-\pi(y)\right| \leq\left(\theta_{P}\right)^{t} \chi_{0}=\left(\theta_{P}\right)^{t} \sqrt{\frac{1}{\pi(x)}-1} \leq \frac{\left(\theta_{P}\right)^{t}}{\sqrt{\pi(x)}} .
$$

Similarly,

$$
\sum_{y \in \mathfrak{X}} \frac{\left|P^{t}(x, y)-\pi(y)\right|}{\sqrt{\pi(y)}} \leq \sqrt{M} \chi_{t} \leq \sqrt{M}\left(\theta_{P}\right)^{t} \chi_{0} \leq \sqrt{M} \frac{\left(\theta_{P}\right)^{t}}{\sqrt{\pi(x)}} .
$$

Proof (Proposition 52). If $f: \mathfrak{X} \rightarrow \mathbb{R}$, denote $D_{f}=\operatorname{diag}(f(x), x \in \mathfrak{X})$. Then, the Boolean cumulant has the following matrix expression:

$$
B^{(r)}\left(Y_{t_{1}}, \ldots, Y_{t_{r}}\right)=\pi D_{f_{t_{1}}}\left(P^{t_{2}-t_{1}}-\mathbf{1} \pi\right) \cdots D_{f_{t_{r-1}}}\left(P^{t_{r}-t_{r-1}}-\mathbf{1} \pi\right) D_{f_{t_{r}}} \mathbf{1},
$$

where 1 is the column vector with all its entries equal to 1 ; see [Fér16, Lemma 10.1]. If we expand this expression as a sum, and denote $Q_{t}=P^{t}-\mathbf{1} \pi$ and $\delta_{i}=t_{i+1}-t_{i}$, then

$$
\begin{aligned}
& B^{(r)}\left(Y_{t_{1}}, \ldots, Y_{t_{r}}\right) \\
& =\sum_{x_{1}, \ldots, x_{r}} \pi\left(x_{1}\right) f_{t_{1}}\left(x_{1}\right) Q_{t_{2}-t_{1}}\left(x_{1}, x_{2}\right) f_{t_{2}}\left(x_{2}\right) \cdots Q_{t_{r}-t_{r-1}}\left(x_{r-1}, x_{r}\right) f_{t_{r}}\left(x_{r}\right)
\end{aligned}
$$

and we obtain

$$
\begin{gathered}
\left|B^{(r)}\left(Y_{t_{1}}, \ldots, Y_{t_{r}}\right)\right| \leq K^{r} \sum_{x_{1}, \ldots, x_{r}} \pi\left(x_{1}\right)\left|Q_{\delta_{1}}\left(x_{1}, x_{2}\right)\right| \cdots\left|Q_{\delta_{r-1}}\left(x_{r-1}, x_{r}\right)\right| \\
\leq K^{r}\left(\theta_{P}\right)^{\delta_{r-1}} \sum_{x_{1}, \ldots, x_{r-1}} \pi\left(x_{1}\right)\left|Q_{\delta_{1}}\left(x_{1}, x_{2}\right)\right| \cdots \frac{\left|Q_{\delta_{r-2}}\left(x_{r-2}, x_{r-1}\right)\right|}{\sqrt{\pi\left(x_{r-1}\right)}} \\
\quad \vdots \\
\leq K^{r} M^{\frac{r-2}{2}}\left(\theta_{P}\right)^{t_{r}-t_{1}} \sum_{x_{1}} \sqrt{\pi\left(x_{1}\right)} \leq K^{r} M^{\frac{r-1}{2}}\left(\theta_{P}\right)^{t_{r}-t_{1}} \cdot
\end{gathered}
$$

Proposition 54. The family of random variables $\left\{Y_{t}\right\}_{t \in \mathbb{N}}$ admits a $K \sqrt{M}$-uniform weighted dependency graph, where, for integers $s<t$, the weight between $Y_{t}$ and $Y_{s}$ is $2\left(\theta_{P}\right)^{t-s}$.

Proof. A lemma of Saulis and Statulevičius [SS91, Lemma 1.1] expresses usual cumulants in terms of Boolean cumulants:

$$
\kappa^{(r)}\left(Y_{t_{1}}, \ldots, Y_{t_{r}}\right)=\sum_{\pi \in \mathcal{P}([r])}(-1)^{|\pi|-1} N(\pi) \prod_{C \in \pi} B^{(|C|)}\left(Y_{t_{j}}, j \in C\right) .
$$

Here, the sum runs over set-partitions $\pi$ of $[r]:=\{1, \ldots, r\} ;|\pi|$ is the number of blocks of a set-partition $\pi$, the product runs over blocks $C$ in $\pi$ and $B^{(|C|)}\left(Y_{t_{j}}, j \in C\right)$ is the Boolean cumulant of the subfamily $\left(Y_{t_{j}}\right)$ indexed by integers $j$ in the block $C$, with the times ordered in increasing order (recall that the Boolean are not symmetric functionals). Finally $N(\pi)$ is a combinatorial factor that can be computed as follows. 
For each block $C$ of $\pi$, denote $m_{C}$ and $M_{C}$ its smallest and biggest elements; then call $n_{C}$ the number of blocks $C^{\prime} \neq C$ such that $m_{C}$ is in the interval $\left[m_{C^{\prime}} ; M_{C^{\prime}}\right]$. We finally define $N(\pi)=\prod_{C \in \pi ; 1 \notin C} n_{C}$. In other terms, $N(\pi)$ counts the functions $g$ mapping each block $C$ of $\pi$ (except the one containing 1) to a block $g(C) \neq C$ such that $m_{C} \in\left[m_{g(C)} ; M_{g(C)}\right]$.

Let us make an observation. If $\pi$ is a partition and $k$ an integer such that each block of $\pi$ either contains only numbers smaller than or equal to $k$ or only numbers bigger than $k$ ( $\pi$ is then said to be disconnected), then no function $g$ as above exists (there is no possible image for the block $C$ containing $k+1$ ) and $N(\pi)=0$. On the other hand, for connected partitions $\pi$, we always have $N(\pi)>0$, so that the sum in (10) is in effect a sum over connected partitions.

Eq. (10) and Proposition 52 imply the bound

$$
\left|\kappa^{(r)}\left(Y_{t_{1}}, \ldots, Y_{t_{r}}\right)\right| \leq(K \sqrt{M})^{r} \sum_{\pi \in \mathcal{P}([r])} N(\pi) \prod_{C \in \pi}\left(\theta_{P}\right)^{t_{M_{C}}-t_{m_{C}}} .
$$

We would like to prove

$$
\left|\kappa^{(r)}\left(Y_{t_{1}}, \ldots, Y_{t_{r}}\right)\right| \leq 2^{r-1}(K \sqrt{M})^{r} \sum_{T \in \mathrm{ST}\left(K_{r}\right)} w(T)
$$

where $w(T)=\prod_{\left\{j, j^{\prime}\right\} \in E_{T}, j<j^{\prime}}\left(\theta_{P}\right)^{t_{j^{\prime}}-t_{j}}$. Therefore it is sufficient for us to find an injective mapping $\eta$ from pairs $(\pi, g)$ as above to edge-bicolored spanning trees $\widetilde{T}$ such that

$$
w(\eta(\pi, g))=\prod_{C \in \pi}\left(\theta_{P}\right)^{t_{M_{C}}-t_{m_{C}}}
$$

here, by convention, the weight $w(\widetilde{T})$ of a colored tree is the weight $w(T)$ of its uncolored version. In the following, we describe such a mapping, concluding the proof of the proposition.

Let $(\pi, g)$ be a pair of objects as above: $\pi$ is a set-partition of $[r]$ and $g$ is function mapping each block $C$ of $\pi$ (except the one containing 1 ) to a block $g(C) \neq C$ such that $m_{C} \in\left[m_{g(C)} ; M_{g(C)}\right]$. For each block $C$ of $\pi$, we consider the set

$$
S(C)=C \cup\left\{m_{C^{\prime}}, C^{\prime} \in g^{-1}(C)\right\}
$$

Let us call $P_{C}$ the path with vertex-set $S(C)$, where the vertices are in increasing order along the path. We also color in blue (resp. in red) edges of this path whose extremity with smaller label is in $C$ (resp. in $\left.\left\{m_{C^{\prime}}, C^{\prime} \in g^{-1}(C)\right\}\right)$.

As an example, take $\pi=\left\{C_{1}, \cdots, C_{6}\right\}$ with $C_{1}=\{1,5,10\}, C_{2}=\{2,11\}, C_{3}=\{3,9\}, C_{4}=\{4,6,13\}$, $C_{5}=\{7,12\}, C_{6}=\{8\}$. As function $g$, we take $g\left(C_{2}\right)=C_{1}, g\left(C_{3}\right)=C_{1}, g\left(C_{4}\right)=C_{2}, g\left(C_{5}\right)=C_{1}$ and $g\left(C_{6}\right)=C_{4}$. In this case, we get $S\left(C_{1}\right)=\{1,2,3,5,7,10\}, S\left(C_{2}\right)=\{2,4,11\}, S\left(C_{4}\right)=\{4,6,8,13\}$ and $S\left(C_{i}\right)=C_{i}$ for $i \in\{3,5,6\}$. The associated bicolored paths are drawn in Fig. 11.

As in Fig. 11, we then take the union of the paths $P_{C_{i}}$, identifying vertices with the same label (the minimum $m_{C} \neq 1$ of a block $C$ appears in the path $S(C)$ and in the path $\left.S(g(C))\right)$. Doing that, we get an edge-bicolored graph that we call $\eta(\pi, g)$. Let us first check that $\eta(\pi, g)$ is a tree. To this purpose, we order the blocks $C_{1}, \ldots, C_{|\pi|}$ of $\pi$ in increasing order of their minima (as done in the example). Observe that this implies that $g\left(C_{i}\right)=C_{j}$ for some $j<i$. We will prove by induction that, for each $i \leq|\pi|$, $P_{C_{1}} \cup \cdots \cup P_{C_{i}}$ is a tree. The case $i=1$ is trivial. For $i>1$, the graph $P_{C_{1}} \cup \cdots \cup P_{C_{i}}$ is obtained by gluing the path $P_{C_{i}}$ on the graph $P_{C_{1}} \cup \cdots \cup P_{C_{i-1}}$, identifying $m_{C_{i}}$ which appears in both. Since $P_{C_{1}} \cup \cdots \cup P_{C_{i-1}}$ is a tree by induction hypothesis, the resulting graph $P_{C_{1}} \cup \cdots \cup P_{C_{i}}$ is a tree as well, concluding the induction. Thus $\eta(\pi, g)=P_{C_{1}} \cup \cdots \cup P_{C_{|\pi|}}$ is a tree.

The equality (11) is easy: since the edge set of $\eta(\pi, g)$ is the union of the edge sets of the $P_{C_{i}}$, we have 

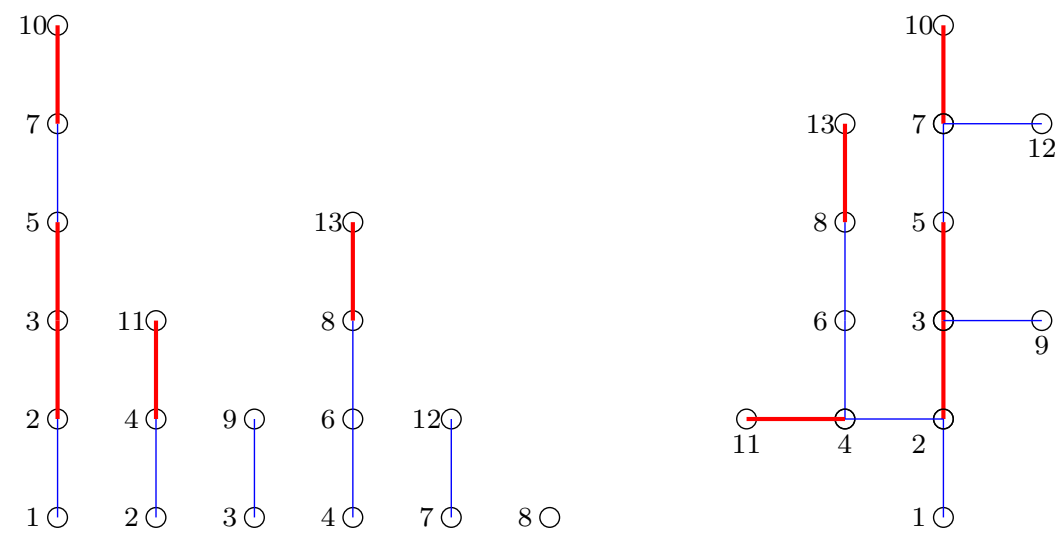

Fig. 11 Illustration of the construction $\eta$ in the proof of Proposition 54: the path $P_{C_{i}}$ and their gluing $\eta(\pi, g)$. For readers of a black-and-white printed version, red edges are thicker.

$$
w(\eta(\pi, g))=\prod_{i=1}^{|\pi|} w\left(P_{C_{i}}\right)=\prod_{i=1}^{|\pi|}\left(\theta_{P}\right)^{t_{M_{C_{i}}}-t_{m_{C_{i}}} .}
$$

We finally need to prove that $\eta$ is injective, i.e. that we can recover $(\pi, g)$ from $\eta(\pi, g)$. We start by the following easy observation: in $\eta(\pi, g)$, vertices with a red incident edge going to a vertex with bigger label are exactly the vertices with a label which is the minimum $m_{C} \neq 1$ of a block $C$ of $\pi$. By construction, such vertices have at most three incident edges, which are as follow:

(E1) as said above, a first one is red and goes to a vertex to bigger label;

(E2) a second one is either blue or red and goes to a vertex of lower label.

(E3) possibly, a last one is blue and goes to a vertex to bigger label (there is such an edge when $m_{C}$ is not alone in its block);

Indeed, in the construction, edges (E1) and (E2) comes from $S_{g(C)}$ while edge (E3) comes from $S_{C}$. We split the vertex $m_{C}$ into two, keeping edges (E1) and (E2) in the same component. Doing that for the $|\pi|-1$ vertices $m_{C} \neq 1$, we inverse the gluing step of the construction of $\eta(\pi, g)$. It is then straightforward to recover $(\pi, g)$.

Theorem 55. Let $\left(X_{t}\right)_{t \in \mathbb{N}}$ be an ergodic Markov chain on a finite state space $\mathfrak{X}$ of size $M$, and $\theta_{P}<1$ the constant associated by (9) with the transition matrix $P$. We consider a sum $S_{n}=\sum_{t=1}^{n} f_{t}\left(X_{t}\right)$ with $\left\|f_{t}\right\|_{\infty} \leq K$. Then, for any $r \geq 1$,

$$
\left|\kappa^{(r)}\left(S_{n}\right)\right| \leq n r^{r-2}\left(2 \frac{1+\theta_{P}}{1-\theta_{P}}\right)^{r-1}(K \sqrt{M})^{r} .
$$

As a consequence:

1. When $\frac{\operatorname{Var}\left(S_{n}\right)}{n^{2 / 3}} \rightarrow+\infty$, we have

$$
d_{\mathrm{Kol}}\left(\frac{S_{n}-\mathbb{E}\left[S_{n}\right]}{\sqrt{\operatorname{Var}\left(S_{n}\right)}}, \mathcal{N}_{\mathbb{R}}(0,1)\right) \leq 76.36\left(\frac{K \sqrt{M}}{\sqrt{\frac{\operatorname{Var}\left(S_{n}\right)}{n}}}\right)^{3}\left(\frac{1+\theta_{P}}{1-\theta_{P}}\right)^{2} \frac{1}{\sqrt{n}},
$$

and in particular, $\frac{S_{n}-\mathbb{E}\left[S_{n}\right]}{\sqrt{\operatorname{Var}\left(S_{n}\right)}}$ converges in law to a standard Gaussian. 
2. This convergence in law happens as soon as $\frac{\operatorname{Var}\left(S_{n}\right)}{n^{\varepsilon}} \rightarrow \infty$ for some $\varepsilon>0$.

Proof. Combining Propositions 54 and 44, the sum $S_{n}$ admits uniform bounds on cumulants with parameters

$$
D_{n}=\left(1+2 \sum_{t=1}^{\infty}\left(\theta_{P}\right)^{t}\right)=\frac{1+\theta_{P}}{1-\theta_{P}}
$$

$N_{n}=n$ and $A=K \sqrt{M}$. Observe that $D_{n}$ is here independent of $n$. We can apply Corollary 30 to get the first part of the theorem. The second follows from Theorem 32.

Remark 56. A bound similar to Eq. (12) is given in [SS91, Theorem 4.19]. We believe however that the proof given there is not correct. Indeed, the proof associates with each partition $\pi$ such that $N(\pi) \neq 0$ a sequence of number $q_{j}$; the authors then claim that "obviously $q_{j} \leq q_{j+1}$ " (p. 93, after eq. (4.53)). This is unfortunately not the case as can be seen on the example of partitions given p. 81 in loc. cit.: for this partition $q_{3}=3$, while $q_{4}=2$. As a consequence of this mistake, the authors forget many partitions $\pi$ when expressing classical cumulants in terms of Boolean cumulants (since they encode partitions with only non-decreasing sequences $q_{i}$ ), which make the resulting bound on classical cumulants too sharp. We have not found a simpler way around this error than the use of uniform weighted dependency graphs presented here. Note nevertheless that our proof still uses several ingredients from [SS91]: the use of Boolean cumulants and the relation between Boolean and classical cumulants.

Remark 5\%. If the functions $f_{t}$ are indicators $f_{t}(x)=1_{x=s_{t}}$, then one can remove the size $(\sqrt{M})^{3}$ in the bound on the Kolmogorov distance. Indeed, in this case, we have

$$
B^{(r)}\left(Y_{t_{1}}, \ldots, Y_{t_{r}}\right)=\pi\left(s_{1}\right) Q_{t_{2}-t_{1}}\left(s_{1}, s_{2}\right) Q_{t_{3}-t_{2}}\left(s_{2}, s_{3}\right) \cdots Q_{t_{r}-t_{r-1}}\left(s_{r-1}, s_{r}\right) .
$$

On the other hand, the individual terms of the matrix $Q_{t}(x, y)$ can be bounded by

$$
\left|Q_{t}(x, y)\right| \leq \sqrt{\frac{\pi(y)}{\pi(x)}}\left(\theta_{P}\right)^{t}
$$

by adapting the proof of Theorem 53. Therefore,

$$
\begin{aligned}
\left|B^{(r)}\left(Y_{t_{1}}, \ldots, Y_{t_{r}}\right)\right| & \leq\left(\theta_{P}\right)^{t_{r}-t_{1}} \pi\left(s_{1}\right) \sqrt{\frac{\pi\left(s_{2}\right)}{\pi\left(s_{1}\right)}} \cdots \sqrt{\frac{\pi\left(s_{r}\right)}{\pi\left(s_{r-1}\right)}} \\
& \leq\left(\theta_{P}\right)^{t_{r}-t_{1}} \sqrt{\pi\left(s_{1}\right) \pi\left(s_{r}\right)} \leq\left(\theta_{P}\right)^{t_{r}-t_{1}}
\end{aligned}
$$

Thus, in this case, one has the bound of Theorem 55 without the factor $(\sqrt{M})^{3}$.

\subsection{The case of linear functionals of the empirical measure}

As a particular case of Theorem 55, one recovers the central limit theorem for linear functionals of empirical measures of Markov chains, that are random variables

$$
Y_{n}=\frac{S_{n}-\mathbb{E}\left[S_{n}\right]}{\sqrt{n}}=\frac{1}{\sqrt{n}} \sum_{t=1}^{n}\left(f\left(X_{t}\right)-\pi(f)\right)
$$

with $f: \mathfrak{X} \rightarrow \mathbb{R}$ fixed function (independent of the time $t$ ). Thus, assuming for instance $\lim _{n \rightarrow \infty} \operatorname{Var}\left(Y_{n}\right)=$ $\Sigma^{2}(f)>0$, we have

$$
d_{\text {Kol }}\left(\frac{S_{n}-\mathbb{E}\left[S_{n}\right]}{\sqrt{\operatorname{Var}\left(S_{n}\right)}}, \mathcal{N}_{\mathbb{R}}(0,1)\right) \leq 77\left(\frac{\|f\|_{\infty} \sqrt{M}}{\Sigma(f)}\right)^{3}\left(\frac{1+\theta_{P}}{1-\theta_{P}}\right)^{2} \frac{1}{\sqrt{n}}
$$


for $n$ large enough. We refer to [Cog72, KV86, Jon04, Häg05] and the references therein for the general background of this Markovian CLT, and to [Bol80, Lez96, Man96] for estimates of the Kolmogorov distance. It seems that we recover some results of [Man96] (see [SC97, §2.1.3]), but we were not able to find and read this paper. In this last paragraph, we discuss the problem of the variance $\operatorname{Var}\left(Y_{n}\right)$, giving sufficient conditions, which are simple to check on examples and ensure $\Sigma^{2}(f)>0$. We also remark that, provided that $\Sigma^{2}(f)>0$, we can also prove complex mod-Gaussian convergence, which implies a zone of normality result and moderate deviation estimates by [FMN16].

Denote $g=f-\pi(f)$, which has expectation 0 under the stationary measure $\pi$. By eventually replacing $f$ with $g$, we can assume that $f$ is centered. The variance of $Y_{n}$ tends to

$$
\Sigma^{2}(f)=\mathbb{E}\left[\left(f\left(X_{0}\right)\right)^{2}\right]+2 \sum_{t=1}^{\infty} \mathbb{E}\left[f\left(X_{0}\right) f\left(X_{t}\right)\right]<+\infty
$$

see [Cog72, Lemma 3.3]. If $\Sigma^{2}(f)>0$, then $\frac{\operatorname{Var}\left(S_{n}\right)}{n^{2 / 3}}=n^{1 / 3} \operatorname{Var}\left(Y_{n}\right) \rightarrow+\infty$ and Theorem 55 applies. Unfortunately, one can easily construct non-trivial examples with $\Sigma^{2}(f)=0$. Thus, consider the Markov chain with 3 states and transition matrix

$$
P=\left(\begin{array}{ccc}
0 & 1 & 0 \\
1 / 2 & 0 & 1 / 2 \\
1 & 0 & 0
\end{array}\right)
$$

it admits for invariant measure $\pi(1)=\pi(2)=\frac{2}{5}$ and $\pi(3)=\frac{1}{5}$. Set $f(1)=1, f(2)=-1$ and $f(3)=0$; one has $\pi(f)=0$, and one computes

$$
\mathbb{E}\left[f\left(X_{0}\right) f\left(X_{k}\right)\right]=\frac{1}{5}\left(\frac{2+\mathrm{i}}{(-1-\mathrm{i})^{k}}+\frac{2-\mathrm{i}}{(-1+\mathrm{i})^{k}}\right) .
$$

It follows that $\Sigma^{2}(f)=0$, although $f$ is non zero.

In the general case of an ergodic Markov chain, fix an element $a$ of the state space $\mathfrak{X}$, and denote $\tau_{a} \geq 1$ the first hitting time of $a$ by the Markov chain, which is almost surely finite and with expectation $1 / \pi(a)$ when starting from $a$. Then, the asymptotic variance $\Sigma^{2}(f)$ can be rewritten as

$$
\Sigma^{2}(f)=\pi(a) \mathbb{E}_{a}\left[\left(\sum_{k=1}^{\tau_{a}} g\left(X_{k}\right)\right)^{2}\right],
$$

see [KS76, Chapter 4]. Therefore, a general condition in order to obtain the bound of Eq. (13) is:

Proposition 58. We have $\Sigma^{2}(f)>0$ if and only if there exists a cycle $\left(x_{1}, \ldots, x_{n}\right)$ in the graph of transitions of the Markov chain such that the sum $\sum_{i=1}^{n} g\left(x_{i}\right)$ of the values of $g=f-\pi(f)$ along this cycle is non-zero. In this case, the bound (13) holds.

The proposition explains readily why the irreducible aperiodic Markov chain

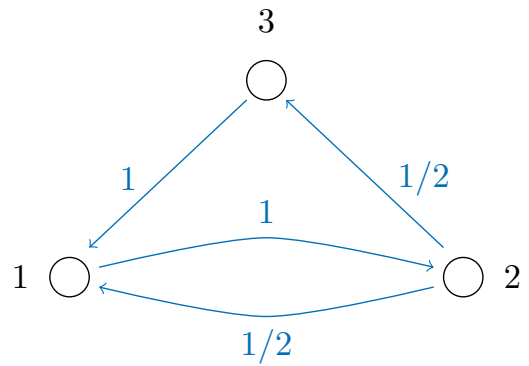

previously studied gives asymptotic variance 0 to the function $f(1)=1, f(2)=-1$ and $f(3)=0$ : the minimal cycles of the chains are $(1,2)$ and $(1,2,3)$, and the sum of the values of $f$ along these cycles is always 0 . 
Another simple criterion to apply Theorem 55 to linear functionals of the empirical measure is:

Proposition 59. Suppose that the ergodic Markov chain is reversible:

$$
\pi(x) P(x, y)=\pi(y) P(y, x)
$$

for any $x, y$. Then, if $f$ is a non-constant function, $\Sigma^{2}(f)>0$ and the bound (13) holds.

Proof. To say that $P$ is reversible is equivalent to the fact that $P$ is a symmetric operator of the Hilbert space $\mathrm{L}^{2}\left(\frac{1}{\pi}\right)$. In particular, $P$ has only real eigenvalues. Besides, the restriction of the operator $I+$ $2 \sum_{k=1}^{\infty} P^{k}$ to the space of functions $f$ with $\pi(f)=0$ is well defined, and it is an auto-adjoint operator on this space with eigenvalues

$$
\frac{1+\lambda_{2}}{1-\lambda_{2}}, \ldots, \frac{1+\lambda_{M}}{1-\lambda_{M}}
$$

where $\lambda_{2} \geq \lambda_{3} \geq \cdots \geq \lambda_{M}$ are the real eigenvalues of $P$ different from 1 . The quantities above are all positive, and larger than $\frac{1-\theta_{P}}{1+\theta_{P}}$ (this value being obtained if $\lambda_{M}=-\theta_{P}$ ). Therefore,

$$
\Sigma^{2}(f)=\left\langle f \mid\left(I+2 \sum_{k=1}^{\infty} P^{k}\right) f\right\rangle_{\mathrm{L}^{2}(\pi)} \geq \frac{1-\theta_{P}}{1+\theta_{P}} \pi\left(f^{2}\right)>0 .
$$

We then obtain

$$
d_{\text {Kol }}\left(\frac{S_{n}-\mathbb{E}\left[S_{n}\right]}{\sqrt{\operatorname{Var}\left(S_{n}\right)}}, \mathcal{N}_{\mathbb{R}}(0,1)\right) \leq 77\left(\frac{\|g\|_{\infty} \sqrt{M}}{\sqrt{\pi\left(g^{2}\right)}}\right)^{3}\left(\frac{1+\theta_{P}}{1-\theta_{P}}\right)^{\frac{7}{2}} \frac{1}{\sqrt{n}}
$$

for $n$ large enough.

Remark 60. In this remark, we discuss mod-Gaussian convergence for linear statistics of Markov chains. We use the above notation and assume that $\Sigma^{2}(f)>0$. Consider the third cumulant of $S_{n}$. One can easily prove that

$$
\rho=\frac{1}{n} \lim _{n \rightarrow \infty} \kappa_{3}\left(S_{n}\right)=\sum_{j, k \in \mathbb{Z}} \kappa\left(f\left(X_{0}, X_{j}, X_{k}\right)\right),
$$

where $\left(X_{t}\right)_{t \in \mathbb{Z}}$ is a bi-infinite stationary Markov chain with transition matrix $P$. (The sum on the righthand side is finite, as consequence of Proposition 54). Let us call $\rho$ this limit. We then consider the rescaled random variables

$$
X_{n}=\left(\frac{S_{n}-\mathbb{E}\left[S_{n}\right]}{\left(\operatorname{Var}\left(S_{n}\right)\right)^{1 / 3}}\right)_{n \in \mathbb{N}} .
$$

As for the magnetization in the Ising model, from [FMN16, Section 5] (with $\alpha_{n}=\operatorname{Var}\left(S_{n}\right)$ and $\beta_{n}=1$ ), we know that $X_{n}$ converges in the mod-Gaussian sense with parameters $t_{n}=\left(\operatorname{Var}\left(S_{n}\right)\right)^{1 / 3}$ and limit $\psi(z)=\exp \left(\frac{L z^{3}}{6}\right)$, with $L=\frac{\rho}{\Sigma^{3}(f)}$. Again, this mod-Gaussian convergence takes place on the whole complex plane and implies a normality zone for $S_{n} / \sqrt{\operatorname{Var}\left(S_{n}\right)}$ of size $o\left(n^{1 / 6}\right)$, see Proposition 4.4.1 in loc. cit.; we also have moderate deviation estimates at the edge of this normality zone, see Proposition 5.2.1. This mod-Gaussian convergence could also have been proved by using an argument of the perturbation theory of linear operators, for which we refer to [Kat80]. Indeed, the Laplace transform of $X_{n}$ writes explicitly as

$$
\mathbb{E}\left[\mathrm{e}^{z X_{n}}\right]=\pi\left(P_{z, f}\right)^{n} 1,
$$

where 1 is the column vector with all its entries equal to $1, \pi$ is the stationary measure of the process, and $P_{z, f}$ is the infinitesimal modification of the transition matrix defined by

$$
P_{z, f}(i, j)=P(i, j) \mathrm{e}^{\frac{z(f(j)-\pi(f))}{\left(\operatorname{Var}\left(S_{n}\right)\right)^{1 / 3}}}
$$

For $z$ in a zone of control of size $O\left(n^{1 / 3}\right)$, one can give a series expansion of the eigenvalues and eigenvectors of $P_{z, f}$, which allows one to recover the mod-Gaussian convergence. The theory of cumulants and weighted dependency graphs allows one to bypass these difficult analytic arguments. 


\section{References}

[AHLV15] O. Arizmendi, T. Hasebe, F. Lehner, and C. Vargas. Relations between cumulants in noncommutative probability. Adv. Math., 282:56 - 92, 2015.

[AS08] N. Alon and J. Spencer. The probabilistic method. Wiley-Interscience Series in Discrete Mathematics and Optimization. Wiley, 2008. 3rd edn.

[Ber41] A. C. Berry. The accuracy of the Gaussian approximation to the sum of independent variates. Trans. Amer. Math. Soc., 49(1):122-136, 1941.

[BHNY08] P. Bourgade, C. Hughes, A. Nikeghbali, and M. Yor. The characteristic polynomial of a random unitary matrix: a probabilistic approach. Duke Math. J., 145:45-69, 2008.

[Bi195] P. Billingsley. Probability and Measure. Wiley Series in Probability and Mathematical Statistics. Wiley, 3rd edition, 1995.

[BKN09] A. D. Barbour, E. Kowalski, and A. Nikeghbali. Mod-discrete expansions. Probab. Th. Rel. Fields, 158(3):859893,2009

[BKR89] A. D. Barbour, M. Karonśki, and A. Rucinśki. A central limit theorem for decomposable random variables with applications to random graphs. J. Combin. Theory Ser. B, 47(2):125-145, 1989.

[Bol80] E. Bolthausen. The Berry-Esseen theorem for functionals of discrete Markov chains. Z. Wahr. verw. Geb., $54: 59-73,1980$.

[Bón10] M. Bóna. On three different notions of monotone subsequences. In Permutation Patterns, volume 376 of London Math. Soc. Lecture Note Series, pages 89-113. Cambridge University Press, 2010.

[BR89] P. Baldi and Y. Rinott. On normal approximations of distributions in terms of dependency graphs. Ann. Probab., 17(4):1646-1650, 1989.

[Bul96] A. V. Bulinskii. Rate of convergence in the central limit theorem for fields of associated random variables. Th. Prob. Appl., 40(1):136-144, 1996.

[CDMN15] R. Chhaibi, F. Delbaen, P.-L. Méliot, and A. Nikeghbali. Mod- $\phi$ convergence: Approximation of discrete measures and harmonic analysis on the torus. arXiv:1511.03922, 2015.

[Cog72] R. Cogburn. The central limit theorem for Markov processes. In Proceedings of the Sixth Annual Berkeley Symposium on Mathematical Statistics and Probability, volume 2, pages 485-512. University of California Press, 1972 .

[DIS73] M. Duneau, D. Iagolnitzer, and B. Souillard. Decrease properties of truncated correlation functions and analyticity properties for classical lattices and continuous systems. Comm. Math. Phys., 31(3):191-208, 1973.

[DIS74] M. Duneau, D. Iagolnitzer, and B. Souillard. Strong cluster properties for classical systems with finite range interaction. Comm. Math. Phys., 35:307-320, 1974.

[DKN15] F. Delbaen, E. Kowalski, and A. Nikeghbali. Mod- $\phi$ convergence. Intern. Math. Res. Not., 2015(11):3445-3485, 2015.

[DS91] P. Diaconis and D. Stroock. Geometric bounds for eigenvalues of Markov chains. Ann. Appl. Probab., 1(1):36-61, 91.

[Ell85] R. Ellis. Entropy, Large Deviations, and Statistical Mechanics. Springer, 1985.

[Fel71] W. Feller. An Introduction to Probability Theory and Its Applications, Volume II. Wiley Series in Probability and Mathematical Statistics. Wiley, 2nd edition, 1971.

[Fér16] V. Féray. Weighted dependency graphs. arXiv preprint 1605.03836, 2016.

[Fil91] J. A. Fill. Eigenvalue bounds on convergence to stationarity for nonreversible Markov chains, with an application to the exclusion process. Ann. Appl. Probab., 1(1):62-87, 1991.

[FMN16] V. Féray, P.-L. Méliot, and A. Nikeghbali. Mod- $\phi$ convergence: Normality zones and precise deviations. SpringerBriefs in Probability and Mathematical Statistics. Springer, 2016.

[FV16] S. Friedli and Y. Velenik. Statistical Mechanics of Lattice Systems: a Concrete Mathematical Introduction. Cambridge University press, 2016. In press.

[Gri92] G. Grimmett. Weak convergence using higher-order cumulants. J. Theoretical Probability, 5(4):767-773, 1992.

[GW16] L. Goldstein and N. Wiroonsri. Stein's method for positively associated random variables with applications to Ising, percolation and voter models. preprint arXiv:1603.05322, to appear in Ann. Inst. Henri Poincaré, 2016.

[Häg05] O. Häggström. On the central limit theorem for geometrically ergodic Markov chains. Probab. Th. Rel. Fields, 132:74-82, 2005.

[Jan88] S. Janson. Normal convergence by higher semi-invariants with applications to sums of dependent random variables and random graphs. Ann. Probab., 16:305-312, 1988.

[JKN11] J. Jacod, E. Kowalski, and A. Nikeghbali. Mod-Gaussian convergence: new limit theorems in probability and number theory. Forum Mathematicum, 23(4):835-873, 2011.

[Jon04] G. L. Jones. On the Markov chain central limit theorem. Probability Surveys, 1:299-320, 2004.

[Kat80] T. Kato. Perturbation Theory for Linear Operators. Classics in Mathematics. Springer-Verlag, 2nd edition, 1980 .

[KN10] E. Kowalski and A. Nikeghbali. Mod-Poisson convergence in probability and number theory. Internat. Math. Res. Not., 18:3549-3587, 2010.

[KN12] E. Kowalski and A. Nikeghbali. Mod-Gaussian distribution and the value distribution of $\zeta\left(\frac{1}{2}+\mathrm{i} t\right)$ and related quantities. J. London Math. Soc., 86(2):291-319, 2012.

[KRT15] K. Krokowski, A. Reichenbachs, and C. Thäle. Discrete Malliavin-Stein method: Berry-Esseen bounds for random graphs, point processes and percolation. arXiv:1503.01029v1 [math.PR], 2015. 
[KS76] J. G. Kemeny and J. L. Snell. Finite Markov Chains. Undergraduate Texts in Mathematics. Springer-Verlag, 1976.

[KS00] J. P. Keating and N. C. Snaith. Random matrix theory and $\zeta\left(\frac{1}{2}+\mathrm{i} t\right)$. Comm. Math. Phys., 214(1):57-89, 2000.

[KS10] V. Y. Korolev and I. G. Shevtsova. On the upper bound for the absolute constant in the Berry-Esseen inequality. Theory of Probability and its Applications, 54(4):638-658, 2010.

[KV86] C. Kipnis and S. R. S. Varadhan. Central limit theorem for additive functionals of reversible Markov processes and applications to simple exclusions. Communications in Mathematical Physics, 104:1-19, 1986.

[Lan93] S. Lang. Real and Functional Analysis, volume 142 of Graduate Texts in Mathematics. Springer-Verlag, 3rd edition, 1993

[Lez96] P. Lezaud. Chernoff-type bound for finite Markov chains. PhD thesis, Université Paul Sabatier, Toulouse, 1996.

[LS59] V. P. Leonov and A. N. Shiryaev. On a method of calculation of semi-invariants. Theory Prob. Appl., 4:319-329, 1959.

[Mal95] P. Malliavin. Integration and Probability, volume 157 of Graduate Texts in Mathematics. Springer-Verlag, 1995.

[Man96] B. Mann. Berry-Esseen central limit theorems for Markov chains. PhD thesis, Harvard University, 1996.

[MM91] V. A. Malyshev and R. A. Minlos. Gibbs random fields. Springer, 1991.

[MN15] P.-L. Méliot and A. Nikeghbali. Mod-Gaussian convergence and its applications for models of statistical mechanics. In In Memoriam Marc Yor - Séminaire de Probabilités XLVII, volume 2137 of Lecture Notes in Mathematics, pages 369-425. Springer-Verlag, 2015.

[New80] C. M. Newman. Normal fluctuations and the FKG inequalities. Comm. Math. Phys., 74(2):119-128, 1980.

[PY05] M. Penrose and J. Yukich. Normal approximation in geometric probability. Stein's Method and Applications, Lecture Note Series, Institute for Mathematical Sciences, National University of Singapore, 5:37-58, 2005.

[Rin94] Y. Rinott. On normal approximation rates for certain sums of dependent random variables. J. Comp. Appl. Math., 55(2):135 - 143, 1994.

[Rud91] W. Rudin. Functional Analysis. McGraw-Hill, 2nd edition, 1991.

[Sat99] K.-I. Sato. Lévy processes and infinitely divisible distributions, volume 68 of Cambridge Studies in Advanced Mathematics. Cambridge University Press, 1999.

[SC97] L. Saloff-Coste. Lectures on finite Markov chains. In Lectures on probability theory and statistics (Saint-Flour, 1996), volume 1665 of Lecture Notes in Mathematics, pages 301-413. Springer-Verlag, 1997.

[Spi58] F. Spitzer. Some theorems concerning 2-dimensional Brownian motion. Trans. Amer. Math. Soc., 87:187-197, 1958.

[SS91] L. Saulis and V. A. Statulevičius. Limit theorems for large deviations, volume 73 of Mathematics and its Applications (Soviet Series). Kluwer Academic Publishers, 1991.

[Sta66] V. A. Statulevičius. On large deviations. Prob. Th. Rel. Fields, 6(2):133-144, 1966.

[Str11] D. W. Stroock. Probability Theory: An Analytic View. Cambridge University Press, 2nd edition, 2011.

[SY83] K.-I. Sato and M. Yamazato. Stationary processes of ornstein-uhlenbeck type. In K. Itô and J. V. Prokhorov, editors, Probability Theory and Mathematical Statistics, Fourth USSR-Japan Symp., volume 1021 of Lecture Notes in Mathematics, pages 541-551. Springer-Verlag, 1983.

[Tao12] T. Tao. Topics in Random Matrix Theory, volume 132 of Graduate Studies in Mathematics. American Mathematical Society, 2012. 